\title{
The properties of the extended warm ionised gas around low-redshift QSOs and the lack of extended high-velocity outflows $\star, \star \star$
}

\author{
B. Husemann ${ }^{1}$, L. Wisotzki ${ }^{1}$, S. F. Sánchez ${ }^{2,3}$, and K. Jahnke \\ ${ }^{1}$ Leibniz-Institut für Astrophysik Potsdam (AIP), An der Sternwarte 16, 14482 Potsdam, Germany \\ e-mail: bhusemann@aip.de \\ 2 Instituto de Astrofísica de Andalucía (CSIC), Camino Bajo de Huétor s/n Aptdo. 3004, 18080-Granada, Spain \\ 3 Centro Astronómico Hispano Alemán de Calar Alto (CSIC-MPIA), 4004 Almería, Spain \\ ${ }^{4}$ Max-Planck-Institut für Astronomie, Königsstuhl 17, 69117 Heidelberg, Germany
}

Received 23 July 2012 / Accepted 28 September 2012

\begin{abstract}
We present a detailed analysis of a large sample of 31 low-redshift, mostly radio-quiet type 1 quasi-stellar objects (QSOs) observed with integral field spectroscopy to study their extended emission-line regions (EELRs). We focus on the ionisation state of the gas, size and luminosity of extended narrow line regions (ENLRs), which corresponds to those parts of the EELR dominated by ionisation from the QSO, as well as the kinematics of the ionised gas. We detect EELRs around 19 of our 31 QSOs (61\%) after deblending the unresolved QSO emission and the extended host galaxy light in the integral field data with a new dedicated algorithm. Based on standard emission-line diagnostics we identify 13 EELRs to be entirely ionised by the QSO radiation, 3 EELRs are composed of $\mathrm{H}$ II regions and 3 EELRs display signatures of both ionisation mechanisms at different locations. The typical size of the ENLR is $\sim 10 \mathrm{kpc}$ at a median nuclear [O III] luminosity of $\log \left(L([\mathrm{O} \mathrm{III]}]) /\left[\mathrm{ergs}^{-1}\right]\right)=42.7 \pm 0.15$. We show that the ENLR sizes are least a factor of $\sim 2$ larger than determined with the Hubble Space Telescope, but are consistent with those of recently reported type 2 QSOs at matching [O III] luminosities. The ENLR of type 1 and type 2 QSOs therefore appear to follow the same size-luminosity relation. Furthermore, we show for the first time that the ENLR size is much better correlated with the QSO continuum luminosity than with the total/nuclear [O III] luminosity. We show that ENLR luminosity and radio luminosity are correlated, and argue that radio jets even in radio-quiet QSOs are important for shaping the properties of the ENLR. Strikingly, the kinematics of the ionised gas is quiescent and likely gravitationally driven in the majority of cases and we find only 3 objects with radial gas velocities exceeding $>400 \mathrm{~km} \mathrm{~s}^{-1}$ in specific regions of the EELR that can be associate with radio jets. In general, these are significantly lower outflow velocities and detection rates compared to starburst galaxies or radio-loud QSOs. This represent a challenge for some theoretical feedback models in which luminous QSOs are expected to radiatively drive an outflow out to scales of the entire host galaxy.
\end{abstract}

Key words. galaxies: active - quasars: emission lines - galaxies: ISM

\section{Introduction}

Extended warm ionised gas with sizes of several tens of kpc around luminous radio-loud quasi-stellar objects (QSOs) was initially discovered by Wampler et al. (1975) and Stockton (1976). Follow-up longslit spectroscopy presented by Boroson \& Oke (1984) and Boroson et al. (1985) confirmed that a substantial part of the extended ionised gas is predominantly photoionised by the hard radiation of the active galactic nucleus (AGN) as indicated by the similar line ratios compared to the compact narrow-line region (NLR). Stockton \& MacKenty (1987) conducted a large ground-based narrow-band imaging survey of 47 luminous QSOs at $z<0.5$ in the [O III] $\lambda 5007$ line (shortly [O III] hereafter) and detected ionised gas beyond $10 \mathrm{kpc}$ around $25 \%$ of the objects, predominantly radio-loud QSOs. These nebulae are often referred to as extended emission line

* Based on observations collected at the Centro Astronómico Hispano Alemán (CAHA) at Calar Alto, operated jointly by the MaxPlanck-Institut für Astronomie and the Instituto de Astrofísica de Andalucía (CSIC).

$\star \star$ Tables 3, 4, and 7 are available in electronic form at http://www. aanda.org regions (EELRs) when their [O III] line luminosity exceeds $5 \times$ $10^{41} \mathrm{erg} \mathrm{s}^{-1}$. A strong alignment effect between the major EELR axis and the radio axis was found for RLQs at $z>0.6$ (McCarthy et al. 1987), suggesting that radio jets have a strong impact on the EELR properties and may directly enhance their brightnesses. Furthermore, EELRs are preferentially found around radio-loud QSOs with a systematically lower broad line region (BLR) metallicity (Fu \& Stockton 2007). Thus, the nature of EELRs and the origin of the gas are not yet clear and remain strongly debated.

The properties of the extended ionised gas around radioquiet QSOs has been studied much less intensively. Bennert et al. (2002) imaged a sample of 7 objects in the [O III] line with the Hubble Space Telescope (HST) and detected ionised gas on scales of up to $10 \mathrm{kpc}$, significantly smaller than the EELR around radio-loud QSOs. Including the NLR sizes of lowluminosity Seyfert galaxies, Bennert et al. (2002) established a correlation between the [O III] luminosity and the size of the NLR, often referred to as the extended NLR (ENLR) when its size exceeds $1 \mathrm{kpc}$. The adopted distinction between the terms ENLR and EELR throughout the paper is that an (E)NLR designates only the AGN photoionised phase of the emission-line 
region, whereas an EELR refer to the entire emission-line region, independently of the ionisation mechanism. Another large HST narrow-band snapshot survey targeting Seyfert galaxies confirmed the existence of such a relation (Schmitt et al. 2003b), but the exact slope and zero-point still remain controversial. Potential caveats of the HST images are the low sensitivity and the lack of spectroscopic information to verify the ionisation mechanism of the gas to be AGN photoionization. For example, longslit spectroscopy of Seyfert galaxies allowed Bennert et al. (2006a,b) to estimate a characteristic distance of $0.7-4 \mathrm{kpc}$ from the nucleus at which the photoionization of star forming region started to dominate against the AGN photoionisation.

The study of extended ionised gas around luminous QSOs is significantly hampered by the severe contamination from the emission of the bright nucleus. Recent studies therefore focused on rare type 2 QSOs for which the emission of the nucleus is thought to be obscured by an optically thick torus following in the Unified Model of AGN (Antonucci 1993). For example, Villar-Martín et al. (2010) detected a huge EELR $(180 \mathrm{kpc})$ around a luminous type 2 QSO $(z=0.399)$ and determined an ENLR size of $\sim 25 \mathrm{kpc}$ from longslit spectra using emission line diagnostics. Furthermore, small samples of type 2 QSOs were recently studied by means of longslit (Greene et al. 2011; VillarMartín et al. 2011b) and integral field spectroscopy (Humphrey et al. 2010). Typical ENLR sizes of $\sim 10 \mathrm{kpc}$ were reported, almost 2 times larger than the ENLRs of unobscured (type 1) QSOs observed with HST.

The extended warm ionised gas around AGN has attracted further attention because it may allow to directly study the impact of an AGN on its surrounding host galaxy. Complex kinematics in the EELR are found around radio-loud QSOs and radio galaxies with line widths up to $1000 \mathrm{~km} \mathrm{~s}^{-1}$ and high velocity clouds that exceed the escape velocity of the galaxy (e.g. McCarthy et al. 1996; Villar-Martín et al. 1999; Tadhunter et al. 2001; Holt et al. 2003, 2006; Nesvadba et al. 2006; Fu \& Stockton 2009). These QSOs are therefore expected to expel a large fraction of gas from the host galaxy. This is usually interpreted as a clear signature for AGN feedback which has been invoked to explain the observed scaling relation between the black hole $(\mathrm{BH})$ mass and host galaxy properties (e.g. Magorrian et al. 1998; Gebhardt et al. 2000; Tremaine et al. 2002; Häring \& Rix 2004; Gültekin et al. 2009), and to efficiently quench star formation. This is required to prevent the excess of massive young galaxies that is often seen in cosmological simulations (e.g. Croton et al. 2006; Khalatyan et al. 2008). Given the short duty cycle of radio-loud AGN and the low radio-loud fraction of the order of $10 \%$, it remains still unclear whether the radio-loud mode of AGN activity can be the primary channel for AGN feedback. Furthermore, the coupling of kinetic jet energy to the ambient medium may be significantly less efficient than currently required by numerical simulation (Holt et al. 2011). Extremely complex extended gas kinematics similar to those in radio-loud counterparts have also been confirmed in individual radio-quiet QSOs like the ultra luminous infrared galaxy (ULIRG) Mrk 231 (Hamilton \& Keel 1987; Lipari et al. 2009; Rupke \& Veilleux 2011; Aalto et al. 2012), but the majority of radio-quiet type 2 QSOs appears to have more quiescent gas kinematics (Humphrey et al. 2010; Greene et al. 2011). An exception are double-peaked [O III] AGN, which were initially thought to be binary AGN candidates, but Fu et al. (2012) reported that the majority of them are simply AGN with a luminous ENLR based on integral-field spectroscopy. The line splitting of several hundred to thousand $\mathrm{km} \mathrm{s}^{-1}$ suggests the presence of either significant outflows driven by the AGN or severe inflows as a result of merging processes. Anyway, the fraction of double-peaked [O III] AGN is $\lesssim 1 \%$ (Wang et al. 2009; Liu et al. 2010; Smith et al. 2010).

Given that type 1 QSOs are much more frequent and allow to infer basic AGN properties from the spectrum of the nucleus, we took the challenge to deal with the deblending of the QSO and extended host galaxy emission. In our early work (Husemann et al. 2008, hereafter Hu08), we studied a sample of 20 mainly radio-quiet QSOs observed with an integral field unit (IFU) and found that 8 of the 20 QSOs are surrounded by an EELR. We emphasised that its presence is linked to the spectral properties of the nucleus itself, in particular the equivalent width (EW) of the Fe II emission and the width of the broad $\mathrm{H} \beta$ line. Since then, we further extended our sample with additional observations of 11 radio-quiet QSOs. In this paper we present a detailed analysis of the EELR ionisation mechanisms, the ENLR size and luminosity as well as the ionised gas kinematics for our entire sample.

The paper is organised as follows. In Sect. 2 we report on the characteristics of the sample including its selection and host galaxy properties based on broad-band imaging. The IFU observations, data reduction and QSO-host deblending are described in Sect. 3. Basic properties of the AGN are determined from the QSO spectra in Sect. 4 and we apply standard emission-line diagnostics to infer the ionisation mechanism of the extended gas in Sect. 5. We describe the properties of the ENLR in Sect. 6 and discuss them in comparison to previous results in the literature. Finally, we present the kinematics of the extended gas in Sect. 7.

Throughout the paper we assume a standard $\Lambda$ CDM cosmological model with $H_{0}=70 \mathrm{~km} \mathrm{~s}^{-1} \mathrm{Mpc}, \Omega_{\mathrm{m}}=0.3$, and $\Omega_{\Lambda}=0.7$.

\section{The QSO sample and characteristics}

\subsection{Sample selection}

Our QSO sample consists of 31 objects in the redshift range $0.06<z<0.3$, which we observed with the Potsdam Multi-Aperture Spectrophotometer (PMAS, Roth et al. 2005) mounted to the Cassegrain focus of the $3.5 \mathrm{~m}$ telescope at the Calar Alto observatory. The QSOs were drawn from two samples that were constructed with different selection criteria. An initial sample of 21 low-redshift QSOs was randomly selected from the Palomar-Green Survey (PG, Schmidt \& Green 1983) and from the Hamburg/ESO survey (HES, Wisotzki et al. 2000) with an absolute magnitude of $M_{B} \leq-23$ (computed with $H_{0}=$ $50 \mathrm{~km} \mathrm{~s}^{-1} \mathrm{Mpc}^{-1}$ ), a commonly used yet arbitrary division line between Seyfert galaxies and QSOs, and a declination of $\delta>$ $-10^{\circ}$ to be observable from the Calar Alto observatory. An additional set of 10 QSOs were drawn from the Sloan Digital Sky Survey (SDSS) DR6 (Adelman-McCarthy et al. 2008). These QSOs were selected to be brighter than $g<17$ within the redshift range $0.13<z<0.3$ and to have a $[\mathrm{O}$ III] $/ \mathrm{H} \beta$ flux density ratio at their peaks greater than 1.5 as measured from the SDSS spectra. This last criterion is based on early results from the initial sample (Hu08) and should give preference to QSOs surrounded by extended ionised gas. The complete list of our observed objects is given in Table 1 including information on some of their main characteristics.

The redshift-luminosity diagram of our observed QSO sample is shown in Fig. 1. We computed absolute magnitudes $\left(M_{B}\right)$ from the apparent $B$ band magnitudes taking into account Galactic foreground extinction (Schlegel et al. 1998) and an appropriate $k$-correction (Wisotzki 2000). The $B$ band magnitudes 
B. Husemann et al.: The properties of the extended warm ionised gas around low-redshift QSOs

Table 1. Characteristics of the QSO sample.

\begin{tabular}{|c|c|c|c|c|c|c|c|c|c|c|}
\hline Name & Alt. name & $\alpha(\mathrm{J} 2000)$ & $\delta(\mathrm{J} 2000)$ & $z^{a}$ & $m_{B}^{b}$ & $M_{B}^{c}$ & $f_{1.4 \mathrm{GHz}}{ }^{d}$ & $f_{5 \mathrm{GHz}}{ }^{e}$ & $R^{f}$ & Class. $^{g}$ \\
\hline PG 0026+129 & & $00^{\mathrm{h}} 29^{\mathrm{m}} 13^{\mathrm{s}} .8$ & $13^{\circ} 16^{\prime} 05^{\prime \prime} 0$ & 0.142 & 14.95 & -24.24 & 7.1 & 5.1 & 1.08 & RI \\
\hline PG $0050+124$ & $\mathrm{I} Z \mathrm{~W} 1$ & $00^{\mathrm{h}} 53^{\mathrm{m}} 34^{\mathrm{s}} .9$ & $12^{\circ} 41^{\prime} 35^{\prime \prime} 0$ & 0.061 & 14.39 & -22.86 & 8.3 & 2.6 & 0.33 & RQ \\
\hline PG 0052+251 & & $00^{\mathrm{h}} 54^{\mathrm{m}} 52^{\mathrm{s}} .2$ & $25^{\circ} 25^{\prime} 39^{\prime} 0$ & 0.155 & 15.42 & -23.97 & 7.1 & 0.7 & 0.24 & $\mathrm{RQ}$ \\
\hline SDSS J0057+1446 & & $00^{\mathrm{h}} 57^{\mathrm{m}} 09^{\mathrm{s}} .9$ & $14^{\circ} 46^{\prime} 10^{\prime}{ }^{\prime} 2$ & 0.172 & 15.58 & -24.05 & 2.4 & $1.3^{\dagger}$ & 0.48 & $\mathrm{RQ}$ \\
\hline HE 0132-0441 & RBS 219 & $01^{\mathrm{h}} 35^{\mathrm{m}} 27^{\mathrm{s}} .0$ & $-04^{\circ} 26^{\prime} 36^{\prime \prime} .2$ & 0.154 & 16.25 & -23.12 & 7.4 & 2.9 & 2.03 & RI \\
\hline SDSS J0155-0857 & & $01^{\mathrm{h}} 55^{\mathrm{m}} 30^{\mathrm{s}} .0$ & $-08^{\circ} 57^{\prime} 04^{\prime \prime} 1$ & 0.165 & 16.83 & -22.71 & $<0.9$ & $<0.5^{\dagger}$ & $<0.58$ & RQ \\
\hline HE 0157-0406 & & $01^{\mathrm{h}} 59^{\mathrm{m}} 49^{\mathrm{s}} .0$ & $-03^{\circ} 52^{\prime} 00^{\prime} .4$ & 0.218 & 17.09 & -23.11 & $<0.9$ & $<0.5^{\dagger}$ & $<0.75$ & RQ \\
\hline PG 0157+001 & Mrk 1014 & $01^{\mathrm{h}} 59^{\mathrm{m}} 50^{\mathrm{s}} \cdot 2$ & $00^{\circ} 23^{\prime} 41^{\prime \prime} 0$ & 0.164 & 15.20 & -24.32 & 26.2 & 8.0 & 2.12 & RI \\
\hline SDSS J0836+4426 & & $08^{\mathrm{h}} 36^{\mathrm{m}} 58^{\mathrm{s}} .9$ & $44^{\circ} 26^{\prime} 02^{\prime \prime} 3$ & 0.254 & 15.54 & -25.03 & 10.0 & $5.3^{\dagger}$ & 1.93 & RI \\
\hline SDSS J0948+4335 & & $09^{\mathrm{h}} 48^{\mathrm{m}} 59^{\mathrm{s}} .5$ & $43^{\circ} 35^{\prime} 19^{\prime} 0$ & 0.226 & 16.81 & -23.48 & $<0.9$ & $<0.5^{\dagger}$ & $<0.56$ & RQ \\
\hline SDSS J1131+2632 & & $11^{\mathrm{h}} 31^{\mathrm{m}} 09^{\mathrm{s}} .2$ & $26^{\circ} 32^{\prime} 07^{\prime \prime} .9$ & 0.244 & 17.41 & -23.06 & 2.7 & $1.4^{\dagger}$ & 2.90 & RI \\
\hline HE $1217+0220$ & PKS $1217+023$ & $12^{\mathrm{h}} 20^{\mathrm{m}} 11^{\mathrm{s}} .9$ & $02^{\circ} 03^{\prime} 41^{\prime \prime} .7$ & 0.239 & 17.23 & -23.20 & 652.0 & 440.0 & 757.18 & RL \\
\hline HE $1226+0219$ & $3 \mathrm{C} 273$ & $12^{\mathrm{h}} 29^{\mathrm{m}} 06^{\mathrm{s}} .7$ & $02^{\circ} 03^{\prime} 07^{\prime \prime} .9$ & 0.158 & 11.42 & -28.01 & 55296.0 & 37000.0 & 302.22 & RL \\
\hline SDSS J1230+6621 & & $12^{\mathrm{h}} 30^{\mathrm{m}} 22^{\mathrm{s}} .2$ & $66^{\circ} 21^{\prime} 54^{\prime \prime} 7$ & 0.184 & 16.75 & -23.05 & $<2.5$ & $<1.3^{\dagger}$ & $<1.46$ & RQ? \\
\hline HE $1228+0131$ & & $12^{\mathrm{h}} 30^{\mathrm{m}} 50^{\varsigma} .0$ & $01^{\circ} 15^{\prime} 21^{\prime \prime} .8$ & 0.117 & 14.82 & -23.92 & 1.4 & 0.2 & 0.04 & RQ \\
\hline SDSS J1230+1100 & & $12^{\mathrm{h}} 30^{\mathrm{m}} 54^{\mathrm{s}} .1$ & $11^{\circ} 00^{\prime} 11^{\prime \prime} .2$ & 0.236 & 16.61 & -23.78 & $<1.0$ & $<0.5^{\dagger}$ & $<0.52$ & RQ \\
\hline PG $1427+480$ & & $14^{\mathrm{h}} 29^{\mathrm{m}} 43^{\mathrm{s}} .1$ & $47^{\circ} 47^{\prime} 27^{\prime \prime} 0$ & 0.221 & 16.33 & -23.90 & $<0.9$ & 0.0 & 0.02 & RQ \\
\hline SDSS J1444+0633 & & $14^{\mathrm{h}} 44^{\mathrm{m}} 14^{\mathrm{s}} .7$ & $06^{\circ} 33^{\prime} 06^{\prime \prime} 8$ & 0.208 & 17.10 & -22.99 & $<1.0$ & $<0.5^{\dagger}$ & $<0.81$ & $\mathrm{RQ}$ \\
\hline HE 1453-0303 & & $14^{\mathrm{h}} 56^{\mathrm{m}} 11^{\mathrm{s}} .5$ & $-03^{\circ} 15^{\prime} 27^{\prime \prime} .8$ & 0.206 & 15.68 & -24.39 & 2.7 & $1.4^{\dagger}$ & 0.58 & $\mathrm{RQ}$ \\
\hline PG $1545+210$ & PKS $1545+210$ & $15^{\mathrm{h}} 47^{\mathrm{m}} 43^{\mathrm{s} .5}$ & $20^{\circ} 52^{\prime} 17^{\prime \prime} .0$ & 0.266 & 16.05 & -24.63 & 1560.0 & 720.0 & 418.15 & RL \\
\hline PG $1612+261$ & TON 256 & $16^{\mathrm{h}} 14^{\mathrm{m}} 13^{\mathrm{s}} .2$ & $26^{\circ} 04^{\prime} 16^{\prime \prime} 0$ & 0.131 & 16.00 & -23.00 & 17.9 & 5.1 & 2.81 & RI \\
\hline SDSS J1655+2146 & & $16^{\mathrm{h}} 55^{\mathrm{m}} 51^{\mathrm{s}} .4$ & $21^{\circ} 46^{\prime} 01^{\prime \prime} 8$ & 0.154 & 16.00 & -23.37 & 6.6 & $3.5^{\dagger}$ & 1.94 & RI \\
\hline PG $1700+518$ & & $17^{\mathrm{h}} 01^{\mathrm{m}} 24^{\mathrm{s}} .9$ & $51^{\circ} 49^{\prime} 21^{\prime \prime} 0$ & 0.292 & 15.43 & -25.48 & 21.6 & 7.2 & 2.36 & RI \\
\hline PG 2130+099 & Mrk 1513 & $21^{\mathrm{h}} 32^{\mathrm{m}} 27^{\mathrm{s}} .8$ & $10^{\circ} 08^{\prime} 19^{\prime} 0$ & 0.061 & 14.62 & -22.63 & 6.0 & 2.0 & 0.32 & RQ \\
\hline HE 2152-0936 & PHL 1811 & $21^{\mathrm{h}} 55^{\mathrm{m}} 01^{\mathrm{s}} .5$ & $-09^{\circ} 22^{\prime} 24^{\prime \prime} .5$ & 0.192 & 14.26 & -25.63 & 1.2 & $0.6^{\dagger}$ & 0.07 & $\mathrm{RQ}$ \\
\hline HE 2158-0107 & & $22^{\mathrm{h}} 01^{\mathrm{m}} 03^{\mathrm{s}} .1$ & $-00^{\circ} 53^{\prime} 01^{\prime \prime} .0$ & 0.213 & 16.69 & -23.45 & 1.6 & $0.8^{\dagger}$ & 0.88 & $\mathrm{RQ}$ \\
\hline HE $2158+0115$ & RBS 1812 & $22^{\mathrm{h}} 01^{\mathrm{m}} 29^{\mathrm{s}} .7$ & $01^{\circ} 29^{\prime} 56^{\prime \prime} 1$ & 0.160 & 16.63 & -22.83 & $<0.9$ & $<0.5^{\dagger}$ & $<0.48$ & $\mathrm{RQ}$ \\
\hline PG 2214+139 & Mrk 304 & $22^{\mathrm{h}} 17^{\mathrm{m}} 11^{\mathrm{s}} .6$ & $14^{\circ} 14^{\prime} 28^{\prime \prime} 0$ & 0.067 & 14.98 & -22.48 & $<2.5$ & 0.2 & 0.05 & RQ \\
\hline HE 2307-0254 & & $23^{\mathrm{h}} 10^{\mathrm{m}} 29.7$ & $-02^{\circ} 38^{\prime} 13^{\prime \prime} .0$ & 0.221 & 16.90 & -23.34 & $<2.5$ & $<1.3^{\dagger}$ & $<1.68$ & RQ? \\
\hline HE 2349-0125 & PKS 2349-014 & $23^{\mathrm{h}} 51^{\mathrm{m}} 56^{\mathrm{s}} .0$ & $-01^{\circ} 09^{\prime} 13^{\prime \prime} .6$ & 0.174 & 16.03 & -23.63 & 1623.2 & 622.7 & 355.46 & $\mathrm{RL}$ \\
\hline HE $2353-0420$ & & $23^{\mathrm{h}} 56^{\mathrm{m}} 32^{\mathrm{s}} .9$ & $-04^{\circ} 04^{\prime} 00^{\prime} 7$ & 0.229 & 17.23 & -23.09 & $<2.5$ & $<1.3^{\dagger}$ & $<2.27$ & RQ? \\
\hline
\end{tabular}

Notes. ${ }^{(a)}$ QSO redshift taken from the PG, HES, or SDSS catalogues. ${ }^{(b)}$ Apparent $B$ band magnitudes taken from the catalogues or synthesised from the SDSS spetrum. ${ }^{(c)}$ Absolute magnitudes $M_{B}$ taking into account Galactic foreground extinction and an appropriate $k$-correction (Wisotzki 2000). ${ }^{(d)}$ Radio fluxes at $1.4 \mathrm{GHz}$ in mJy as measured by the NVSS all-sky survey or the FIRST survey when available. ${ }^{(e)}$ Radio fluxes at 5 GHz in mJy taken from Kellermann et al. (1989) and an unpublished catalog (Gopal-Krishna et al., priv. comm.), if available. Values marked with $\dagger$ were estimated from the radio flux at $1.4 \mathrm{GHz}$ assuming a spectral index of $\alpha=-0.5$. ${ }^{(f)}$ Ratio $R$ of the $5 \mathrm{GHz}$ radio to optical flux density at $4400 \AA$. The optical flux density $f_{v}(4400 \AA)$ in mJy was estimated from the optical $B$ band magnitude with the relation $m_{B}=-2.5 \log f_{v}(4400 \AA)-48.36$ (Schmidt \& Green 1983). ${ }^{(g)}$ Radio classification of the QSO following Kellermann et al. (1989) into radio loud (RL) and radio quiet (RQ) and radio intermediate (RI) QSOs. An unambigous classification for a few object cannot be made due to upper limits in $R$.

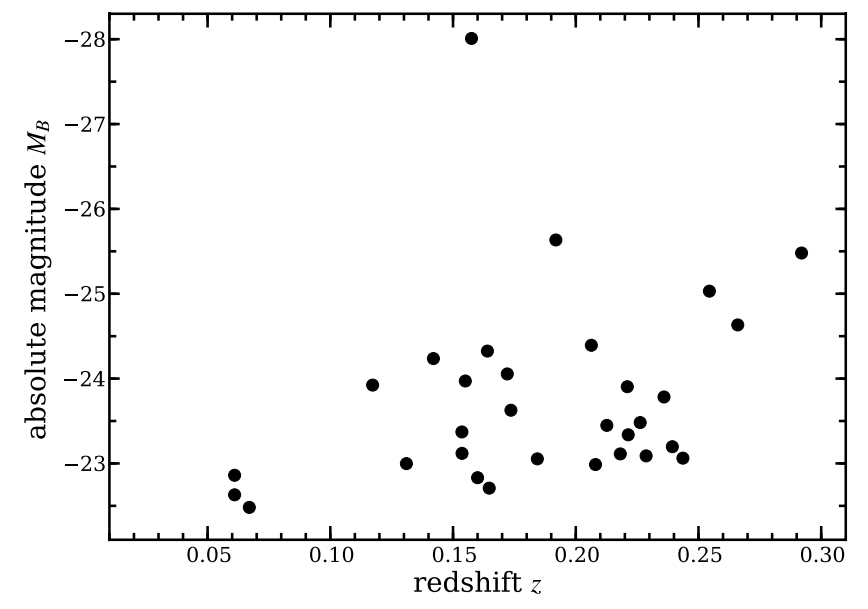

Fig. 1. Absolute $B$ band magnitude against the redshift for all objects in our QSO sample.

and redshifts were either taken from the available catalogues or directly measured from publicly available SDSS spectra. The absolute magnitudes are in the range of $-26<M_{B}<-22.3$ with a median value of -23.45 . The median redshift of the sample is $z=0.184$. Thus, the majority of our objects are luminous low-redshift QSOs.

\subsection{Radio properties}

Radio-loud and radio-quiet QSOs (RLQs and RQQs, respectively) are often distinguished based on the $R$ parameter, which is defined as the ratio of the radio (at $6 \mathrm{~cm} / 5 \mathrm{GHz}$ ) to the optical flux density (at $4400 \AA$ ). We adopt the classification of Kellermann et al. (1989), who separated RQQs with $R<1$, RLQs with $R>10$ and a few radio-intermediate objects in between the two classes. Not every object of our sample has been observed at $5 \mathrm{GHz}$, but the NRAO VLA Sky Survey (NVSS Condon et al. 1998) and the Faint Images of the Radio Sky at Twenty-centimetres (FIRST) survey (Becker et al. 1995) provide radio measurements at $1.4 \mathrm{GHz}(21 \mathrm{~cm})$ for a large fraction of the sky with a completeness level of $2.5 \mathrm{mJy}$ and $1 \mathrm{mJy}$, respectively. We adopt a power-law dependence of the radio flux 
Table 2. Available radio imaging data for some RQQs.

\begin{tabular}{|c|c|c|c|c|c|}
\hline Name & $v^{a}$ & $\mathrm{Beam}^{b}$ & $\mathrm{Size}^{c}$ & $\mathrm{PA}^{d}$ & Reference \\
\hline PG $0026+129$ & $8.4 \mathrm{GHz}$ & 0.35 & 0.60 & $\cdots$ & (1) \\
\hline PG $0052+251$ & $4.8 \mathrm{GHz}$ & 0.59 & $<0 \prime^{\prime} 09$ & & (1) \\
\hline Mrk 1014 & $8.4 \mathrm{GHz}$ & 0.36 & $2{ }^{\prime \prime} 6$ & $90^{\circ}$ & (1) \\
\hline PG $1612+261$ & $8.4 \mathrm{GHz}$ & 0.28 & $1 . .0$ & $66^{\circ}$ & (2) \\
\hline PG $1700+518$ & $8.4 \mathrm{GHz}$ & 0.28 & 0.5 & $-42^{\circ}$ & (2) \\
\hline PG $1700+518$ & $1.6 \mathrm{GHz}$ & $0 .{ }^{\prime} 02$ & 0.5 & $-41^{\circ}$ & (3) \\
\hline PG 2130+099 & $4.8 \mathrm{GHz}$ & 0.35 & 2.9 & $-48^{\circ}$ & (1) \\
\hline
\end{tabular}

Notes. ${ }^{(a)}$ Observing frequency $v$ of the radio observations. ${ }^{(b)}$ Beam size of the interferometric radio observations. ${ }^{(c)}$ Maximum size of the extended radio emission. ${ }^{(d)}$ Position angle of the major radio axis. $90^{\circ}$ points to the East and $0^{\circ}$ to the North.

References. (1) Leipski et al. (2006); (2) Kukula et al. (1998); (3) Yang et al. (2012).

densities $\left(f_{v} \propto v^{\alpha_{\mathrm{r}}}\right)$ with a spectral index of $\alpha_{\mathrm{r}}=-0.5$, at the border line between steep- and flat-spectrum radio sources, to estimate the flux density at $5 \mathrm{GHz}$ from measurements at $1.4 \mathrm{GHz}$ when required. Only 4 objects in the sample can be classified as RLQs, 8 objects have intermediate radio properties, and the majority of objects within the sample are clearly RQQs.

Besides the integrated radio measurements, archival high spatial resolution images for 6 of our RQQs were obtained with the VLA interferometer at subarcsecond resolution and were presented by Kukula et al. (1998) and Leipski et al. (2006). We retrieved the processed images from the VLA image archive ${ }^{1}$ and some have also been kindly provided by the authors. The characteristics of these images are listed in Table 2. All except PG 0052+251 display extended radio emission with sizes up to $3^{\prime \prime}$. A triple radio source as an unambiguous sign of a jet was identified in the RQQs Mrk 1014, in PG 2130+099 using the VLA, and in PG $1700+518$ using the higher spatial resolution of the European VLBI network (Yang et al. 2012).

\subsection{QSO host morphologies}

\subsubsection{Available broad-band imaging}

High spatial resolution broad-band images were obtained with the HST for most of the PG QSOs over the last decade, except PG 1427+490, PG 1612+261 and PG 2214+139. These HST images were initially published in various unrelated articles (see Table 3), but a homogeneous analysis of all the public data was presented by Kim et al. (2008). Ground-based broad-band images are additionally available for all QSOs covered by the SDSS footprint. The SDSS survey images with an effective exposure time of $54 \mathrm{~s}$ were usually obtained at a seeing around $\sim 1$ '. $2(r)$. Yet, it has so far not been attempted to uncover the underlying QSO host galaxies in these images given the short exposure time and the low spatial resolution of the images.

No broad-band images have so far been presented for most of the HES QSOs in our sample. Ground-based $H$ band images were obtained for a few QSOs in 2003 with the SOFI IR spectrograph and imaging camera (Moorwood et al. 1998) mounted on the New Technology Telescope (NTT) and are publicly available in the ESO archive, which we reduced with the corresponding standard ESO pipeline. Additionally, we obtained $R$ band images for three HES QSOs with the $60 \mathrm{~cm}$ telescope of the Lightbuckets robotic telescope network (hereafter LB). This

\footnotetext{
1 https://archive.nrao.edu/archive/archiveimage.html
}

small telescope is equipped with an Apogee Alta U42 CCD camera providing a spatial resolution of $0{ }^{\prime} 57 \mathrm{pixel}^{-1}$. With an exposure time of $\sim 1 \mathrm{~h}$, these images are nominally deeper than SDSS, but suffer from a seeing of $z 2^{\prime \prime}$. We reduced these images following the standard procedures including bias subtraction, flatfielding and combination of the dithered exposures with custom Python scripts.

\subsubsection{QSO-host deblending}

In order to perform a consistent analysis of the available broadband images we deblended the QSO and host galaxy component using GALFIT (Peng et al. 2002, 2010). We emphasise that our main aim here is only to recover the stellar light surface brightness distribution of the underlying host galaxies and distinguish between bulge-dominated and disc-dominated host galaxies whenever possible. A more detailed analysis of the images would be beyond our scope and hardly possible given the low spatial resolution of many images.

We created an empirical point spread function (PSF) from bright stars in the field close to the target. Dedicated PSF stars were observed with HST only for a few objects, so that we relied on synthetic PSFs generated with the TinyTim software package (Krist 1995). First, we used GALFIT to model each object with a PSF component only, to check whether any significant residuals of a host galaxy could actually be detected. In a second step, we modelled the images with a superposition of a Sersić light profile (Sersic 1968) for the host and a PSF for the QSO component. Two examples of the deblending results for the ground-based SDSS image are presented in Fig. 2.

Each QSO host was modelled with three different radial Sersić surface brightness profiles: 1) Sersić index fixed to $n=1$ (exponential profile), 2) Sersić index fixed to $n=4$ (de Vaucouleurs profile), or 3) Sersić variable as a free parameter. The resulting $\chi^{2}$ values and the best-fit structural parameters are reported in Table 3 . The superposition of a bulge and a disc model provides a significantly better fit to the surface brightness distribution of PG $2130+099$ and I Zw 1 than a single component. The structural parameters reported in Table 3 for these two QSO hosts refer to the disc component of the model.

The QSO hosts observed with HST are all well resolved and we recover the host galaxies also for the majority of the groundbased observations. PSF-subtracted host galaxy images are presented below in Fig. 7 for all objects. The ground-based images clearly suffer from their low spatial resolution, so that the models are strongly affected by PSF mismatches in compact galaxies. Such mismatches sometimes lead to non-physically high Sersić indices. The comparison of the SDSS $i$ and $r$ images allow us to judge the robustness of the results. Strongly different results indicate that the structural parameters are not well constrained. Furthermore, a close apparent companion affected the modelling of SDSS J1655+2146, so that a meaningful model could only be inferred for the host galaxy with a fixed Sersić index.

\subsubsection{Morphological classification}

Discriminating between a bulge- and a disc-dominated QSO host galaxy is not straightforward, even for the high spatial resolution images obtained with HST. A commonly used criterion is the best-fitting Sersić index $n$, where $n>2.5$ indicates a bulge-dominated and $n<2.5$ indicates a disc-dominated systems (e.g. Sánchez et al. 2004b; Bell et al. 2004). Alternatively, the lowest $\chi^{2}$ value is computed for a bulge and a disc-model to 


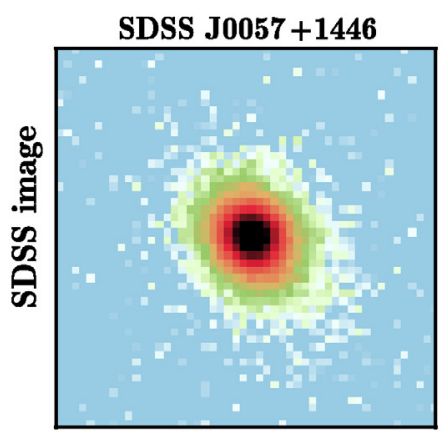

SDSS J0836 +4426
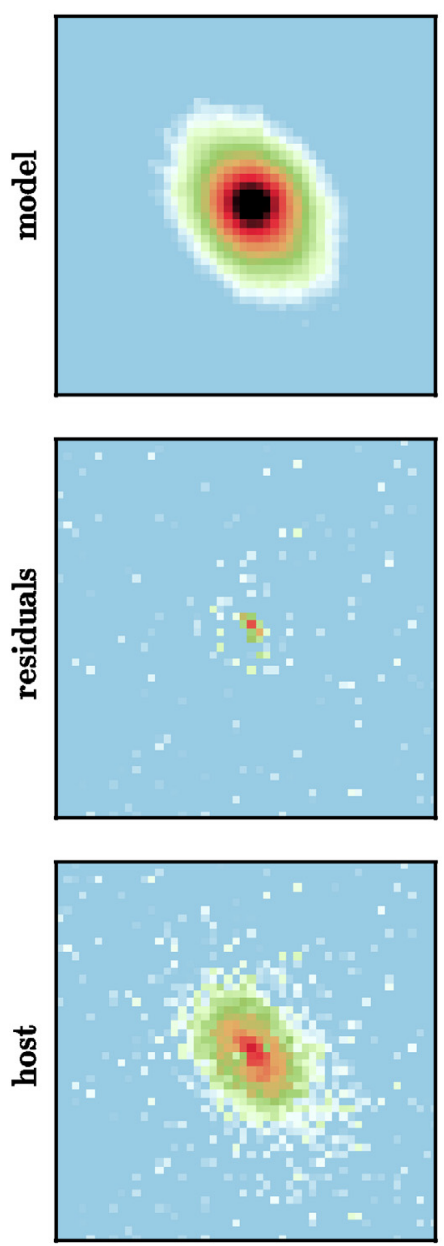
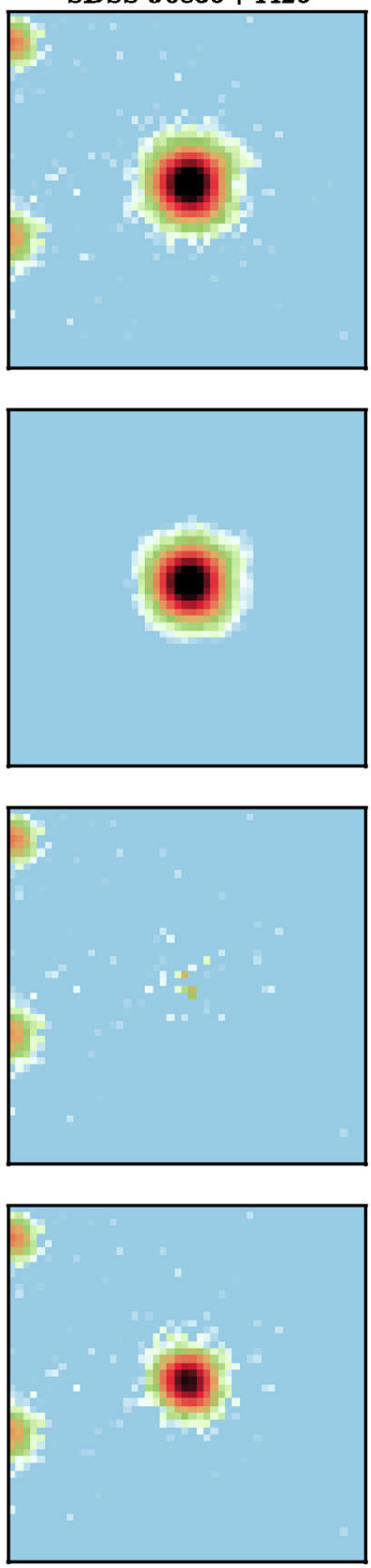

Fig. 2. Two examples of the QSO-host deblending process for SDSS $i$ band images. The PSF was constructed from a bright nearby star in the field. A best-fitting Sersić surface brightness model plus a PSF for the nucleus was obtained with GALFIT and subtracted from the original SDSS image to highlight the residuals. The host galaxy is recovered after subtracting the best-fit PSF component from the original image with nucleus-to-host ratios of $\sim 3.5$ and $\sim 2.2$ for SDSS J0057+1446 and SDSS J0836+4426, respectively.

identify the best matching model (e.g. McLeod \& McLeod 2001; Sánchez \& González-Serrano 2003; Dunlop et al. 2003; Jahnke \& Wisotzki 2003; Guyon et al. 2006). However, the statistical significance of the favoured model compared to other options needs to be considered. On the other hand, a visual classification of the host is often preferred to identify spiral structures of discdominated systems, or apparently disturbed merging system.

For the HST images, the $\chi^{2}$ values robustly discriminate between the models. We adopt the criterion by Guyon et al. (2006), which demands a significant difference in the reduced $\chi^{2}$ of $\left|\chi_{v}^{2}(n=1)-\chi_{v}^{2}(n=4)\right|>0.05$. The $\chi^{2}$ classification always agreed with the one based solely on the best-fit Sersic index and we achieved good agreement with literature results. The situation is more difficult for the ground-based observations, where the $\chi^{2}$ values sometimes differ only marginally. A visual inspection of the images, together with large axis ratios $(b / a)$, may provide evidence in some cases for disc-dominated hosts even when a bulge-dominated model was formally preferred as in the cases of RBS 219, PG 1612+261, PHL 1811 and HE 2353-0420. When the best-fitting Sersić index is $n>7$, we doubt that any reliable classification can be made, because flux transfer likely happened between the PSF and the host galaxy component. In the other cases, we refer to the best-fitting Sersic indices for our classification, but consider these classifications as tentative.

From the 28 QSOs with available broad-band imaging, we classified 17 host galaxies into 5 bulge-dominated, 8 discdominated and 4 ongoing merging galaxies. 5 of the remaining hosts are likely bulge-dominated systems and one is probably disc-dominated. For the rest of the host galaxies we were not able to constrain the morphology. Our sample seems to be almost evenly split up into bulge- and disc-dominated systems without any preference. A similar balance of morphological types were also reported for completely different samples of QSO host galaxies (e.g. Jahnke et al. 2004; Cisternas et al. 2011).

\section{Spatially resolved spectroscopy of QSOs}

\subsection{PMAS IFU observations}

IFU observations for the initial sample were carried out with PMAS in the nights from the 2th to 7th of April in 2002 and from the 2nd to 4th of May 2003. The QSOs of the extended sample were observed in the nights from the 13th to 17th of March and from the 5th to 8th of September in 2008. In all cases we used the PMAS lens-array with the highest magnification providing a seeing-limited sampling of $0.5 \times 0.5$ with a field of view (FoV) of $8^{\prime \prime} \times 8^{\prime \prime}$ for the 256 spaxels $^{2}$. The host galaxies of the observed QSOs effectively fill the FoV in most cases (see Fig. 7), but are significantly larger for the three nearest objects $(z<0.10)$ and a few extended systems at higher redshift.

The low resolution V300 grating was used for the observations of the initial QSO sample covering a large wavelength range including the major diagnostic spectral features around the $\mathrm{H} \beta$ and $\mathrm{H} \alpha$ emission lines simultaneously. Higher spectral resolution observations with the V600 grating were taken for the extension of the sample, but then centred on the $\mathrm{H} \beta$ emission line only. For both setups we estimated the spectral resolution from the O I $\lambda 5577$ night-sky emission line to be $6.1 \AA$ and $3.2 \AA$ Full-Width at Half Maximum (FWHM), respectively. At least two exposures were usually taken for each QSO with individual integration times in the range of $900 \mathrm{~s}$ up to $2400 \mathrm{~s}$ reaching a total integration time of $1-2 \mathrm{~h}$ per object. Table 4 summarises the details of the observations including notes on the ambient observing conditions.

A set of arc lamp ( $\mathrm{HgNe})$ and continuum lamp exposures were acquired before or after the target frames for fibre tracing and wavelength calibration. Skyflats were taken during twilight to measure the variations in the fibre-to-fibre transmission, and spectro-photometric standard stars were regularly observed at the beginning and at the end of the nights with the same instrumental setups as the QSOs.

\footnotetext{
Spectral pixel, a single spatial resolution element containing a spectrum along the entire wavelength range.
} 


\subsection{Data reduction}

The basic data reduction was performed with the R3D reduction package (Sánchez 2006) designed to reduce fibre-fed integral field spectroscopy data. We used the independent modular tasks of the package for the following basic reduction steps: 1. bias subtraction, 2 . fibre tracing on the continuum lamp exposure, 3. extraction of the fibre spectra, 4. wavelength calibration with the arc-lamp exposures, 5. fibreflat correction based on observed skyflats, 6. flux calibration of the spectra using spectrophotometric standard star observations, and 7. creation of a datacube from the row-stacked spectra.

Prior to the reduction we detected and removed cosmic rays hits from the individual raw frames with the DCR routine (Pych 2004) that was designed originally for long-slit spectroscopic data. We used a conservative configuration of DCR such that night sky lines or emission-line features of our target itself were not removed. A few prominent cosmic ray hits inevitably remained in the data given the imperfection of the algorithm for IFU data. Thus, we always required that emission lines must be spatially extended, that they have a line width equal or greater than the instrumental resolution, and that the doublet line ratios are consistent with their theoretical values.

As discussed in Roth et al. (2005), the PMAS instrument suffers from flexure depending on the hour angle of the observations. This inevitably leads to shifts between different exposures of the same target in dispersion and/or cross-dispersion direction of the spectra on the charge-coupled device (CCD) detector. Flexure shifts were measured with the IRAF task imalign by comparing the position of $\sim 20$ sky emission lines distributed over the whole CCD in the raw image. When only one set of calibration frames (continuum+arc lamp) was available for a sequence of observations, we resampled the calibration frames to match with position of the individual exposures. The science frame taken right after or before the calibration frames was used as the reference for the zero position.

A median sky spectrum was created from spaxels containing no emission lines from the host galaxy and subsequently subtracted from the whole datacube. Given that several QSO hosts fill the entire PMAS FoV we accepted a contamination of the sky spectrum by stellar continuum emission in these cases. This has no strong impact on our emission line analysis, but the Balmer emission lines need to be corrected for the underlying stellar absorption lines. How the inaccurate sky subtraction affects our emission-line measurements will be addressed in Sect. 3.4.

The datacubes were then corrected for differential atmospheric refraction (DAR) by resampling each monochromatic slice such that the position of the QSO is aligned to a common reference point at all wavelengths. All standard star observations were used to create a mean sensitivity function for each run and instrument setup, so that the datacubes are properly flux calibrated taking into account the airmass and the atmospheric extinction law at the Calar Alto observatory (Sánchez et al. 2007). Finally, we combined the datacubes using either a variance weighted mean or a clipping method whenever 3 or more exposures were available to reject remaining cosmic rays and artefacts.

Most of the data were taken under clear or photometric conditions. In order to check the quality and uncertainty of the spectrophotometry, we compared the flux-calibrated QSO spectra with available SDSS spectra taking into account the size of SDSS fibres. The deviation between the QSO spectra in the continuum over the whole wavelength range was $\leq 20 \%$, which we adopt as our systematic spectrophotometric uncertainty.

\subsection{QSO-host deblending with $Q D e b l e n d^{3 D}$}

Similar to the deblending of the broad-band images we need to decompose the QSO and host emission also in our IFU data to study and interpret the emission of the host galaxy without the QSO contamination. The three-dimensional nature of the IFU date requires new analysis techniques and software. We developed the software tool QDeblend ${ }^{3 \mathrm{D}}$ for this particular task ${ }^{3}$. At the core of the program is an iterative deblending algorithm which is a significant improvement of a simple technique introduced by Christensen et al. (2006).

The basic concept is to treat the spaxels of the IFU datacube as a set of independent spectra rather than as a sequence of monochromatic images. In the absence of atmospheric dispersion the spectrum of a point source would then be the same in each spaxel, multiplied by a scale factor according to the PSF. In the case of QSO observations, the scale factors of the PSF can be directly determined from the strength of the broad emission lines with respect to the adjacent continuum as described by Jahnke et al. (2004). Here we outline the iterative algorithm implemented in QDeblend ${ }^{3 \mathrm{D}}$. A more detailed explanation with illustrative diagrams of the iterative process can be found in the user manual of QDeblend ${ }^{3 \mathrm{D}}$.

In a first step a high $\mathrm{S} / \mathrm{N}$ nuclear QSO spectrum is extracted from the datacube. After a PSF has been determined from a broad QSO emission line, the nuclear QSO spectrum is subtracted from each spaxel after multiplied by the corresponding PSF scale factor. However, significant over-subtraction will occur when the nuclear QSO spectrum is contaminated by host galaxy light. In QDeblend ${ }^{3 D}$ we minimize this host galaxy contamination by iteratively estimating a mean host galaxy spectrum from the residual datacube that is subsequently subtracted from the QSO spectrum. A rectangular aperture and surrounding annulus is used as a default to extract QSO and host galaxy spectra, but any set of spaxels for the QSO and host galaxy spectrum could be specified. Because the host galaxy becomes brighter towards the galaxy centre, the mean host spectrum from the annulus should be scaled in brightness before it is subtracted from the initial nuclear QSO spectrum. That scale factor is unconstrained from the IFU data itself. One may either adopt an a-priori defined analytic surface brightness profile, a constant surface brightness, or a manually set scale factor which fulfils a certain criterion. The iteration is stopped when the decontaminated nuclear QSO spectrum converges.

For our PMAS spectra we use the broad $\mathrm{H} \alpha$ or $\mathrm{H} \beta$ lines of the QSOs to perform a QSO-host deblending with QDeblend ${ }^{3 \mathrm{D}}$. Because we focus on the emission lines of the ionised gas around $\mathrm{H} \beta$ and $\mathrm{H} \alpha$ region in this work, we restrict the QSO-host deblending to this narrow wavelength regions around the two Balmer lines. This almost entirely avoids the problem of PSF mismatch due to the wavelength dependence of the atmospheric seeing. The nuclear QSO spectra are taken from the brightest spaxel in the data, and the surrounding 8 spaxels are used to estimate a mean host galaxy spectrum from the residuals. Here we assume a constant surface brightness to extrapolate the host galaxy emission towards the central spaxel. The QSO spectra converged already after four iterations at which we stopped the iterative process.

\subsection{Imperfect sky subtraction and Balmer absorption}

One problem with our PMAS IFU observations is the imperfect subtraction of the sky background. Because no blank sky

Available for download at http://sf.net/projects/qdeblend/ 
B. Husemann et al.: The properties of the extended warm ionised gas around low-redshift QSOs

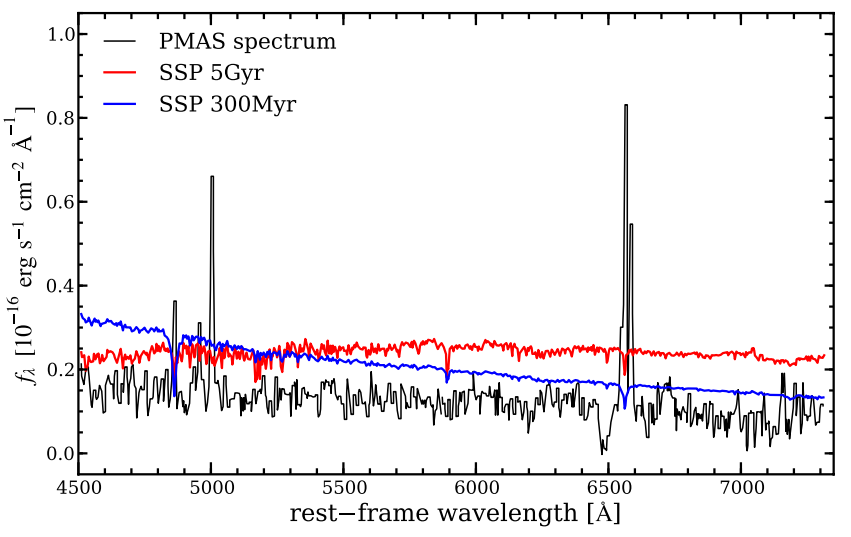

Fig. 3. Comparing the PMAS spectra of PKS 2349-014 (region A) with two SSP model spectra matched to the HST photometry. The systematic offset between the SSP models and the PMAS spectra is caused by an over-subtraction of the sky background in the PMAS observations.

fields were observed together with the target, we were forced to estimate the sky directly from the target datacubes itself. We used spatial regions free of extended emission lines to avoid any over-subtraction of these lines, but those regions may contain significant emission from stellar continuum if the host galaxy extends across the PMAS FoV. Thus, the stellar continuum is over-subtracted in several cases for which we were unable to reliably infer the "real" stellar continuum from our data.

Our PMAS observations were primarily targeted to map the emission lines of the ionised gas. While it would be also interesting to study the stellar populations of the QSO hosts from their continuum emission, we find that given our exposure times, the $\mathrm{S} / \mathrm{N}$ is generally too low to quantitatively interpret the stellar absorption patterns. Nevertheless, the measured strength of the Balmer emission lines will depend on the depth of the underlying stellar absorption, which we take into account when using the Balmer lines for diagnostic purposes. In order to explore the impact of the Balmer absorption line on our measurements, we combined synthetic single stellar population (SSP) models from Bruzual \& Charlot (2003) with the absolute surface brightness photometry of the HST broad-band images. In a first step, we convolved the nucleus-subtracted HST images with the PSF of the corresponding PMAS observation and rebinned the image to the spatial sampling of our PMAS data. Afterwards, we scaled a suite of SSP spectra to the surface brightness in several specific regions. In Fig. 3 we compare two SSP models (300 Myr and 5 Gyr) matched to the HST photometry with the PMAS spectrum of PKS 2349-014 (region A as defined in Fig. 7) as an example. The PMAS spectrum is well below both SSP model spectra, which can be entirely attributed to the over-subtracted sky background.

The strengths of the Balmer absorption lines depend on the age of the stellar population. We measured the differences between the $\mathrm{H} \beta$ emission line fluxes of the continuum subtracted and unsubtracted PMAS spectra expressed as $\Delta \log (\mathrm{H} \beta)=$ $\log \left(\mathrm{H} \beta_{\text {sub. }}\right)-\log \left(\mathrm{H} \beta_{\text {unsub. }}\right)$ for 18 SSP spectra with ages of the order of $300 \mathrm{Myr}<t_{\mathrm{SSP}}<5 \mathrm{Gyr}$ assuming solar metallicity. We show the dependence of $\Delta \mathrm{H} \beta$ on $t_{\mathrm{SSP}}$ for PKS 2349-014 (region A) as an example in Fig. 4. We find that the $\mathrm{H} \beta$ emission line flux is underestimated due to the corresponding stellar absorption line by $0.07-0.2$ dex in this case of PKS 2349-014. The redshift of that object $(z=0.174)$ is close to the median redshift of our sample and representative for the majority of objects. The $\mathrm{H} \beta$ emission-line flux is more severely affected for the

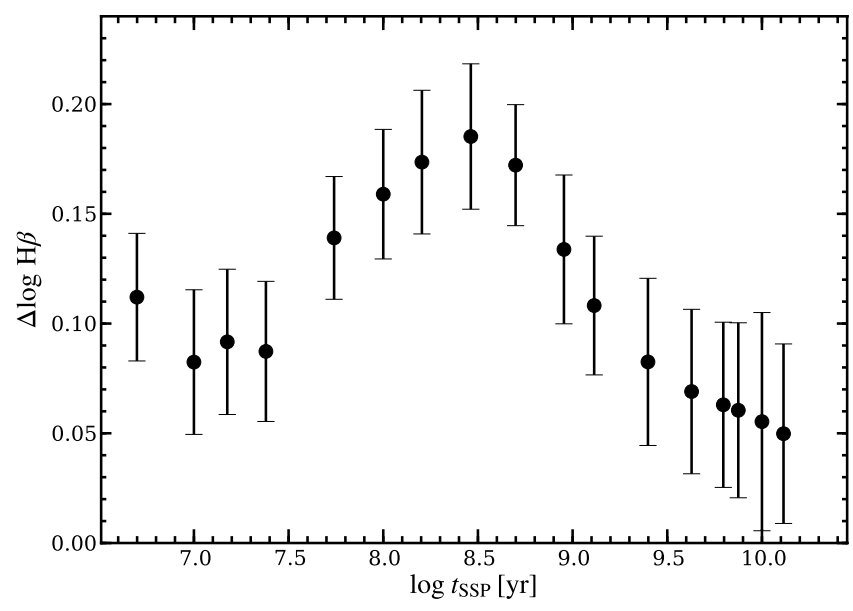

Fig. 4. Underestimation of the $\mathrm{H} \beta$ emission line flux $(\Delta \log (\mathrm{H} \beta))$ due to the stellar absorption line as a function of the stellar population age for the PMAS spectrum of PKS 2349-014 (region A).

few object at $z=0.06$ given the lower equivalent width of their emission line.

Because of the unknown age of the stellar population, we used the average of $\Delta \log (\mathrm{H} \beta)$ taken over all SSP ages with an error corresponding to the possible range of values. For the host that fill the PMAS FoV and were not observed with HST, we corrected all $\mathrm{H} \beta$ lines by an average factor of $\Delta \log (\mathrm{H} \beta)=$ $0.13 \pm 0.08 \mathrm{dex}$, determined from all objects with reliable HST photometry. Although the $\mathrm{H} \alpha$ emission line is also affected by the stellar absorption, we made no correction given that the correction factor $(0.03 \pm 0.01 \mathrm{dex})$ is negligible for our purposes.

\section{AGN parameters from the QSO spectra}

Our PMAS data provide high S/N spectra of the QSOs without any wavelength-dependent slit losses, because the IFU covers the entire seeing disc of a point source even under bad seeing conditions. We use those spectra to derive some fundamental AGN parameters, like redshifts, black hole masses $M_{\mathrm{BH}}$, and Eddington ratios $\lambda=L_{\mathrm{bol}} / L_{\mathrm{Edd}}$. The contamination of the QSO spectrum by host galaxy light was removed or at least minimised by our QSO-host deblending process described above. For a few QSOs where the SDSS spectra had significantly higher $\mathrm{S} / \mathrm{N}$ we used these instead. From the SDSS broad-band images we estimated a host contamination of $\$ 15 \%$ in the SDSS spectra and corrected the continuum luminosities correspondingly.

\subsection{Spectral modelling of the $\mathrm{H} \beta-[\mathrm{OIII}]$ region}

All of the AGN parameters of interest were calibrated to spectral features around the broad $\mathrm{H} \beta$ line. This wavelength region is bracketed by two adjacent Fe II complexes as shown in Fig. 5 (left panel). Most studies of QSO spectra (e.g. Boroson \& Green 1992; Greene \& Ho 2005; Woo et al. 2006; McGill et al. 2008) used a template generated from the proto-typical QSO I Zw 1 (Boroson \& Green 1992; Véron-Cetty et al. 2004) to subtract the Fe II complexes over a large wavelength range. We cannot apply this Fe II template fitting method here in a consistent manner because most of our QSO spectra do not reach enough to the blue to reliably estimate the underlying power-law continuum. We also note that the two available Fe II templates significantly disagree (Fig. 5 left panel). However, the dominant Fe II emission in the proximity of $\mathrm{H} \beta$ and [O III] originates in both cases 

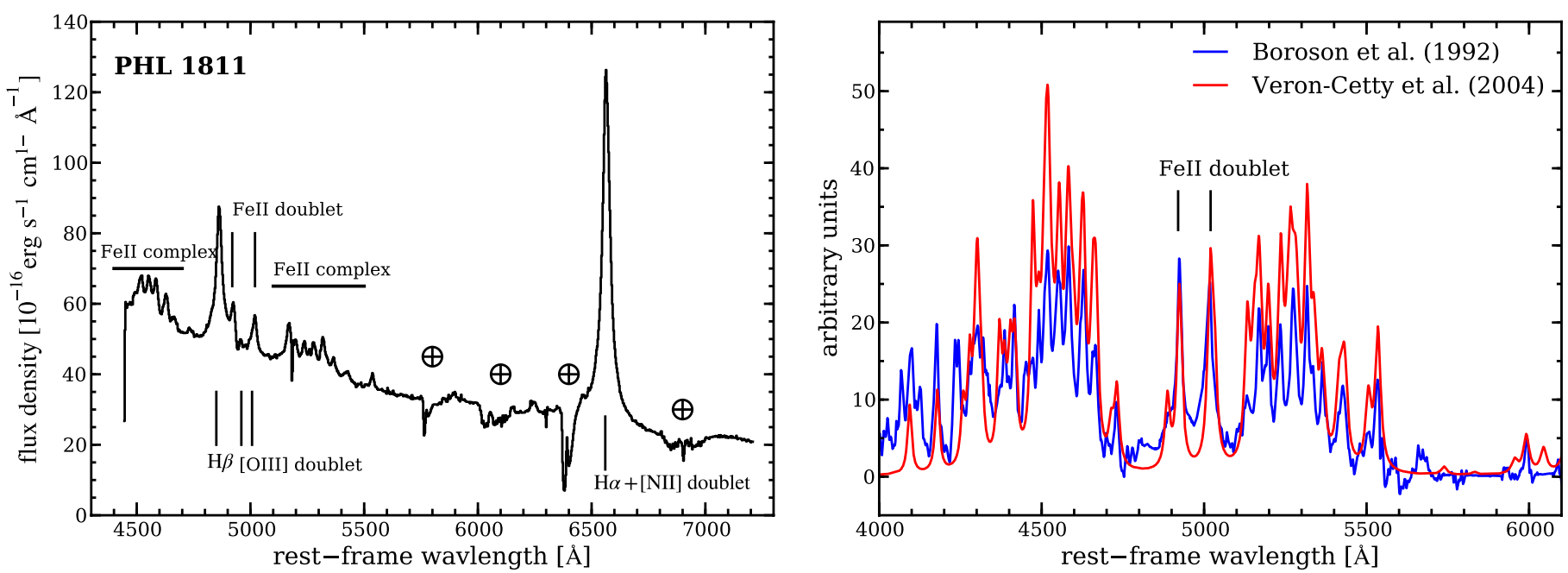

Fig. 5. Left panel: QSO spectrum of PHL 1811 observed with PMAS as a representative example for a strong Fe II emitter. The available wavelength range of the V300 grating was set such that $\mathrm{H} \beta$ and $\mathrm{H} \alpha$ would be covered at the redshift of the QSO. The Fe II complex blueward of $\mathrm{H} \beta$ is not fully covered for PHL 1811, as in many other objects. Right panel: comparison of two available Fe II templates from Boroson \& Green (1992) and Véron-Cetty et al. (2004) both based on the proto-typical QSO I Zw 1. Both templates were scaled to match the isolated Fe II $\lambda 5014$ line. The disagreement between the templates is severe given that they were generated from the same QSO.

from two isolated Fe II lines, enabling us to choose a more simpler approach to handle the Fe II $\lambda \lambda 4929,5018$ lines over a restricted wavelength range.

We thus modelled only the wavelength region of 4700-5100 , which encompasses $\mathrm{H} \beta$, [O III] and almost pure continuum regions on either side. A superposition of Gaussians was used to represent the line profile of the broad and narrow emission lines including the two prominent Fe II $\lambda \lambda 4929,5018$ lines. The full model consists of up to 3 Gaussian components for the $\mathrm{H} \beta$ line, up to 2 Gaussian components for the profile of the [O III] and Fe II doublets and a straight line to approximate the local continuum. The two lines of a doublet are coupled as a system in line width, radial velocity and line ratio (a factor of 3 for [O III] $\lambda \lambda 4960,5007$ and 1.29 for Fe II $\lambda \lambda 4924,5018)$, which significantly increases the robustness of our model and reduces the number of free parameters. If a narrow $\mathrm{H} \beta$ component is present on top of a broad $\mathrm{H} \beta$ emission profile, we couple its kinematic parameters with that of the narrow [O III] component as these lines originate both from the NLR. In cases where the Fe II lines were broad and their width could hardly be constrained against the background continuum, we coupled the line width and radial velocity to that of the broad $\mathrm{H} \beta$ line.

The best-fitting parameters were found by searching for the lowest $\chi^{2}$ of the residuals with a downhill simplex algorithm (e.g. Press et al. 1992). Two representative examples of these models are presented in Fig. 6. From the best-fitting model, we measured several quantities including the continuum flux at $5100 \AA\left(f_{5100}\right)$ and the width of the broad $\mathrm{H} \beta$ component expressed both in terms of FWHM $\left(\mathrm{FWHM}_{\mathrm{H} \beta}\right)$ and line dispersion $\left(\sigma_{\mathrm{H} \beta}\right)$. These values are listed in Table 5 for the entire sample. To check whether these measurements are robust compared to a full Fe II template fitting approach we performed a simple consistency check. We generated artificial QSO spectra from our best-fitting models excluding the Fe II components but adding the Boroson \& Green iron template matched to the Fe II $\lambda 5014$ line strength and broadened to the FWHM of the broad $\mathrm{H} \beta$ line. These artificial spectra were subsequently analysed in exactly the same way as the original QSO spectra. The recovered values for the FWHM of $\mathrm{H} \beta$ and the continuum flux at $5100 \AA$ differ by
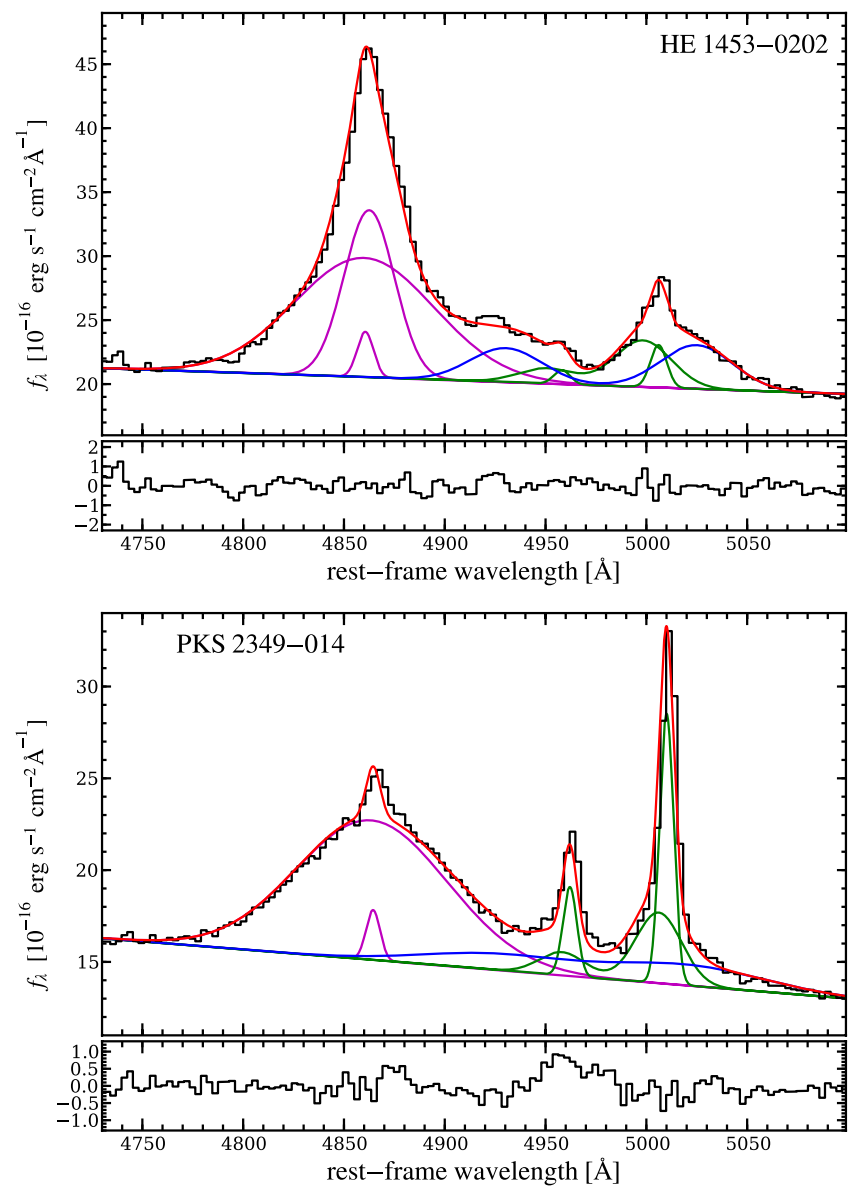

Fig. 6. Spectral modelling of the $\mathrm{H} \beta-[\mathrm{O} I \mathrm{III}]$ region for two representative QSO spectra. The black line correspond to the observed QSO spectra and the red curve represents our best fit model. The model consists of individual Gaussian components for the emission lines $(\mathrm{H} \beta$-magenta, [O III]-green, Fe II-blue) above a straight line for the local continuum. The residual of the model are shown in a dedicated panel below.

only $0.02 \mathrm{dex}$ in the mean, with a maximum offset of $0.05 \mathrm{dex}$ and 0.08 dex, respectively. 
B. Husemann et al.: The properties of the extended warm ionised gas around low-redshift QSOs

Table 5. AGN parameters measured or derived from the QSO spectrum.

\begin{tabular}{|c|c|c|c|c|c|c|c|c|}
\hline Object & {$\left[\begin{array}{c}f_{\mathrm{NLR},[\mathrm{O} \mathrm{III}]} \\
{\left[10^{-16} \frac{\mathrm{erg}}{\mathrm{scm}^{2}}\right]}\end{array}\right.$} & $\begin{array}{c}\left.\log L_{\mathrm{NLR},[\mathrm{O}} \mathrm{III}\right] \\
{\left[\mathrm{erg} \mathrm{s}^{-1}\right]}\end{array}$ & $\left.\begin{array}{c}f_{5100} \\
{\left[10^{-16} \frac{\mathrm{erg}}{\mathrm{scm}^{2} \AA}\right.}\end{array}\right]$ & $\begin{array}{l}\log L_{5100} \\
{\left[\mathrm{erg} \mathrm{s}^{-1}\right]}\end{array}$ & $\begin{array}{c}F W H M_{\mathrm{H} \beta} \\
{\left[\mathrm{km} \mathrm{s}^{-1}\right]}\end{array}$ & $\begin{array}{c}\sigma_{\mathrm{H} \beta} \\
{\left[\mathrm{km} \mathrm{s}^{-1}\right]}\end{array}$ & $\begin{array}{c}\log \left(M_{\mathrm{BH}}\right) \\
{\left[M_{\odot}\right]}\end{array}$ & $\log \lambda$ \\
\hline PG $0026+129$ & $505 \pm 3$ & $42.54 \pm 0.09$ & $27.5 \pm 6.7$ & $44.96 \pm 0.11$ & $2430 \pm 28$ & $3471 \pm 18$ & 9.0 & -1.2 \\
\hline I Zw 1 & $1667 \pm 13$ & $42.23 \pm 0.09$ & $45.8 \pm 11.3$ & $44.31 \pm 0.11$ & $1111 \pm 12$ & $1652 \pm 10$ & 8.0 & -0.9 \\
\hline PG $0052+251$ & $667 \pm 2$ & $42.69 \pm 0.09$ & $25.1 \pm 5.8$ & $44.98 \pm 0.10$ & $4604 \pm 24$ & $2088 \pm 8$ & 8.6 & -0.7 \\
\hline SDSS J0057+1446 & $203 \pm 14$ & $42.27 \pm 0.12$ & $18.6 \pm 4.2$ & $44.95 \pm 0.10$ & $10506 \pm 159$ & $4143 \pm 39$ & 9.1 & -1.3 \\
\hline HE $0132-0441$ & $200 \pm 33$ & $2.16 \pm 0.16$ & $12.6 \pm 2.9$ & $44.69 \pm 0.10$ & $1487 \pm 21$ & $960 \pm 47$ & 7.7 & -0.2 \\
\hline HE 0157-0406 & $82 \pm 2$ & $2.08 \pm 0.10$ & $6.2 \pm 1.3$ & $44.72 \pm 0.09$ & $5493 \pm 174$ & $2333 \pm 77$ & 8.5 & -1.0 \\
\hline SDSS J0155-0857 & $211 \pm 11$ & $2.23 \pm 0.11$ & $6.7 \pm 1.4$ & $44.47 \pm 0.09$ & $6650 \pm 87$ & $2824 \pm 35$ & 8.6 & -1.2 \\
\hline Mrk 1014 & $901 \pm 10$ & $2.85 \pm 0.09$ & $13.7 \pm 3.0$ & $44.77 \pm 0.09$ & $3002 \pm 25$ & $1549 \pm 16$ & 8.2 & -0.6 \\
\hline SDSS J0846+4426 & $1038 \pm 53$ & $43.34 \pm 0.11$ & $17.9 \pm 3.9$ & $45.35 \pm 0.10$ & $4169 \pm 128$ & $1771 \pm 56$ & 8.6 & -0.4 \\
\hline SDSS J0948+4335 & $179 \pm 5$ & $2.45 \pm 0.10$ & $5.4 \pm 1.2$ & $44.72 \pm 0.09$ & $4060 \pm 61$ & $1571 \pm 30$ & 8.2 & -0.6 \\
\hline SDSS J1131+2623 & $252 \pm 33$ & $42.68 \pm 0.14$ & $3.4 \pm 0.7$ & $44.59 \pm 0.09$ & $9370 \pm 328$ & $3957 \pm 128$ & 8.9 & -1.5 \\
\hline PKS $1217+023$ & $281 \pm 2$ & $42.71 \pm 0.09$ & $14.6 \pm 3.2$ & $45.20 \pm 0.10$ & $4300 \pm 178$ & $2272 \pm 686$ & 8.7 & -0.7 \\
\hline 3 C 273 & $996 \pm 32$ & $42.87 \pm 0.10$ & $272.7 \pm 59.2$ & $46.04 \pm 0.09$ & $2852 \pm 7$ & $1999 \pm 7$ & 9.1 & -0.2 \\
\hline SDSS J1230+1100 & $241 \pm 7$ & $42.64 \pm 0.10$ & $5.1 \pm 1.1$ & $44.73 \pm 0.10$ & $4439 \pm 129$ & $2307 \pm 30$ & 8.5 & -0.9 \\
\hline HE $1228+0131$ & $454 \pm 27$ & $42.23 \pm 0.11$ & $44.5 \pm 9.7$ & $44.95 \pm 0.09$ & $1671 \pm 11$ & $1572 \pm 38$ & 8.3 & -0.5 \\
\hline SDSS J1230+6621 & $447 \pm 7$ & $42.65 \pm 0.09$ & $5.9 \pm 1.2$ & $44.54 \pm 0.09$ & $3297 \pm 93$ & $2190 \pm 22$ & 8.4 & -1.0 \\
\hline PG $1427+480$ & $240 \pm 5$ & $2.55 \pm 0.10$ & $5.3 \pm 1.1$ & $44.67 \pm 0.09$ & $2455 \pm 68$ & $1268 \pm 105$ & 8.0 & -0.4 \\
\hline SDSS J1444+0633 & $447 \pm 7$ & $2.65 \pm 0.09$ & $5.9 \pm 1.2$ & $44.54 \pm 0.09$ & $3297 \pm 93$ & $2190 \pm 22$ & 8.4 & -1.0 \\
\hline HE 14 & $195 \pm 13$ & $2.53 \pm 0.12$ & $27.4 \pm 7.9$ & $45.31 \pm 0.12$ & $2513 \pm 43$ & 1795 & 8.6 & -0.4 \\
\hline PKS $1545+21$ & $286 \pm 2$ & $2.84 \pm 0.09$ & $14.1 \pm 3.1$ & $45.29 \pm 0.10$ & $5644 \pm 38$ & $2397 \pm 15$ & 8.8 & -0.7 \\
\hline PG $1612+261$ & $2591 \pm 17$ & $43.14 \pm 0.09$ & $21.2 \pm 5.1$ & $44.74 \pm 0.11$ & $2770 \pm 26$ & $1177 \pm 11$ & 7.9 & -0.4 \\
\hline SDSS J1655+2146 & $687 \pm 7$ & $42.71 \pm 0.09$ & $12.0 \pm 3.0$ & $44.65 \pm 0.11$ & $2483 \pm 37$ & $1576 \pm 27$ & 8.1 & -0.6 \\
\hline PG $1700+518$ & $145 \pm 5$ & $42.62 \pm 0.10$ & $15.6 \pm 3.4$ & $45.43 \pm 0.09$ & $2067 \pm 41$ & $2600 \pm 53$ & 9.0 & -0.7 \\
\hline PG 2130+099 & $870 \pm 15$ & $41.98 \pm 0.09$ & $48.3 \pm 11.1$ & $44.40 \pm 0.10$ & $2727 \pm 10$ & $2144 \pm 17$ & 8.3 & -1.0 \\
\hline HE 2152-0936 & $122 \pm 7$ & $42.17 \pm 0.11$ & $60.7 \pm 13.8$ & $45.59 \pm 0.10$ & $1877 \pm 13$ & $1853 \pm 16$ & 8.8 & -0.3 \\
\hline HE 2158-0107 & $241 \pm 1$ & $42.59 \pm 0.09$ & $9.6 \pm 2.3$ & $44.89 \pm 0.11$ & $5486 \pm 45$ & $2325 \pm 19$ & 8.6 & -0.9 \\
\hline HE $2158+0115$ & $124 \pm 1$ & $41.92 \pm 0.09$ & $4.8 \pm 1.1$ & $44.21 \pm 0.10$ & $2739 \pm 33$ & $1637 \pm 42$ & 7.9 & -0.9 \\
\hline PG 2214+139 & $466 \pm 11$ & $41.78 \pm 0.10$ & $48.3 \pm 12.1$ & $44.44 \pm 0.11$ & $5506 \pm 23$ & $3190 \pm 77$ & 8.6 & -1.4 \\
\hline HE $2307-0254$ & $61 \pm 3$ & $42.00 \pm 0.11$ & $8.6 \pm 1.9$ & $44.89 \pm 0.10$ & $2172 \pm 27$ & $1823 \pm 32$ & 8.4 & -0.7 \\
\hline PKS 2349-014 & $279 \pm 7$ & $42.40 \pm 0.10$ & $14.0 \pm 3.0$ & $44.85 \pm 0.09$ & $4041 \pm 83$ & $2265 \pm 25$ & 8.6 & -0.9 \\
\hline HE $2353-0420$ & $224 \pm 1$ & $42.58 \pm 0.09$ & $2.7 \pm 0.6$ & $44.42 \pm 0.10$ & $4176 \pm 40$ & $1774 \pm 17$ & 8.1 & -0.9 \\
\hline
\end{tabular}

\subsection{Black hole masses and Eddington ratios}

Black hole masses can be estimated from single epoch broadline AGN spectra using the so-called virial method (Peterson \& Wandel 2000; Vestergaard 2002). It combines the empirically derived size-luminosity relation of the BLR as determined via reverberation mapping (e.g. Kaspi et al. 2000; Peterson et al. 2004; Kaspi et al. 2005; Bentz et al. 2006c, 2009) and the kinematics of the BLR estimated from the width of the broad emission-lines assuming virial motion of the clouds. We adopted the empirical relation between the BLR size and the continuum luminosity at $5100 \AA\left(L_{5100}\right)$ calibrated by Bentz et al. (2009), and the prescription of Collin et al. (2006) to infer the kinematics of the broad line clouds from the $\mathrm{H} \beta$ line dispersion $\left(\sigma_{\mathrm{H} \beta}\right)$. A virial scale factor of 3.85 was estimated by Collin et al. (2006) to match the virial $\mathrm{BH}$ masses with the $M_{\mathrm{BH}}-\sigma_{*}$ relation of local inactive galaxies (Tremaine et al. 2002). This yields a black hole mass calibration of

$M_{\mathrm{BH}}=10^{7.41}\left(\frac{\sigma_{\mathrm{H} \beta}}{1000 \mathrm{~km} \mathrm{~s}^{-1}}\right)^{2}\left(\frac{L_{5100}}{10^{44} \mathrm{erg} \mathrm{s}^{-1}}\right)^{0.52} M_{\odot}$.

The error on the estimated $\mathrm{BH}$ masses using the virial method is dominated by systematic errors rather than measurement errors from high S/N QSO spectra (see Denney et al. 2009). In addition to the intrinsic uncertainties in the adopted scaling relations and the adopted line profile model, the unknown geometry and kinematics of the BLR and the correct determination of the QSO continuum flux are sources of uncertainty. The BH masses estimated in this way (see Table 5) are assumed to be accurate within a factor of $\sim 2(\sim 0.3 \mathrm{dex})$.

Furthermore, we computed the bolometric luminosity of the QSOs adopting a standard bolometric correction of

$L_{\mathrm{bol}}=9 \times \lambda L_{\lambda}(5100 \AA)$,

following Kaspi et al. (2000) which is in agreement with the estimates of Richards et al. (2006). From the BH masses we also calculated the Eddington luminosities $L_{\text {Edd }}$ and Eddington ratios $\lambda \equiv L_{\mathrm{bol}} / L_{\mathrm{Edd}}$. The derived values for the $M_{\mathrm{BH}}$ and $\lambda$ are given in Table 5. Almost all of our QSOs have black hole masses of $M_{\mathrm{BH}} \gtrsim 10^{8} M_{\odot}$ and Eddington ratios in between $0.1 \lesssim \lambda<1$. None of our QSOs accretes above the Eddington limit, which is in agreement with the results of low-redshift QSO studies (e.g. Schulze \& Wisotzki 2010; Steinhardt \& Elvis 2010). The median $\mathrm{BH}$ mass and median Eddington ratio for our sample are $M_{\mathrm{BH}} \simeq$ $4 \times 10^{8} M_{\odot}$ and $\lambda \simeq 0.15$. In individual cases, $M_{\mathrm{BH}}$ are found to be 0.5 dex larger than previous estimates based on the FWHM of $\mathrm{H} \beta$ (e.g. Vestergaard \& Peterson 2006), but the values based on $\sigma_{\mathrm{H} \beta}$ should be more robust as argued by Peterson et al. (2004) and Collin et al. (2006).

\section{Emission line diagnostic of extended ionised gas}

\subsection{Mapping of extended emission and their spectra}

After subtracting the QSO contribution with QDeblend ${ }^{3 \mathrm{D}}$ from the observed data we study the properties of ionised gas around 
Table 6. Surface brightness limits $(3 \sigma)$ for HST [O III] narrow-band images.

\begin{tabular}{lccc}
\hline \hline Name & $\begin{array}{c}\text { HST } \\
{\left[10^{-15} \mathrm{erg} \mathrm{s}^{-1} \mathrm{~cm}^{-2} \mathrm{arcsec}^{-2}\right]}\end{array}$ \\
\hline PG 0026+129 & 10.73 & 3.79 & 0.14 \\
PG 0052+251 & 2.77 & 0.80 & 0.18 \\
Mrk 1014 & 2.74 & 0.91 & 0.21 \\
\hline
\end{tabular}

Notes. ${ }^{(a)}$ A median filter of $5 \times 5$ pixel box size was used.

the QSOs. Firstly, we extract $40 \AA$ wide narrow-band images centred on the [O III] and $\mathrm{H} \alpha$ lines of the QSO after removing any continuum emission estimated the adjacent bands. The synthetic passband of our narrow-band images roughly correspond to $\pm 900 \mathrm{~km} \mathrm{~s}^{-1}$ in the rest frame at the median redshift of the sample, so that extended emission even with high velocities would be captured. We detect extended emission in [O III] around 16 of 31 QSOs ( 50\%), of which a high fraction have also detectable $\mathrm{H} \alpha$ emission (when covered by our observations). Whether the apparent [O III] emission around 3C 273 is a real feature is currently unclear as discussed below. Additionally, $\mathrm{H} \alpha$ emission is detected around three objects without noticeable [O III] emission, I Zw 1, RBS 219 and PHL 1811.

In Fig. 7 we present the PMAS narrow-band images of all objects for which we detected extended ionised gas in comparison to the nucleus-subtracted broad-band host images, when available. The ionised gas generally does not extend beyond the size of the host galaxies at the surface brightness limits of our observations. The only exception is HE 2158-0107 for which the ionised gas region is much more extended than the host galaxy and reaches even beyond the PMAS FoV with a projected size of $>22 \mathrm{kpc}$. A detailed discussion and interpretation of this interesting QSO is presented in Husemann et al. (2011) and beyond the scope of this paper.

As the $\mathrm{H} \beta, \mathrm{H} \alpha$, and [N II] lines are rather weak in individual spaxels we decided to co-add several spaxels to increase their $\mathrm{S} / \mathrm{N}$. Based on the morphology of the extended emission we defined up to 4 characteristic spatial regions, avoiding spaxels with obvious signs of artefacts or QSO residuals that could affect the emission line measurements. The region boundaries are shown in Fig. 7 together with the corresponding co-added spectra in the rest-frame wavelength intervals 4800-5100 ̊ and 6450-6750 A, if covered by our observation. The $\mathrm{H} \beta$ and [O III] emission lines were clearly detected in most of these objects. Essentially no contamination of the broad $\mathrm{H} \beta$ emission was left in these extended spectra, which demonstrates the robustness of the deblending process at least down to 0.' 5 distance from the QSO position. The $\mathrm{H} \alpha$ and [N II] lines are often redshifted into one of the atmospheric absorption bands, which prevent a detection of these lines in those cases.

To quantitatively measure the observed emission line properties, we modelled each line in the spectra with a Gaussian profile taking into account the theoretically fixed line ratios of the [O III] $\lambda \lambda 4960,5007$ and [N II] $\lambda \lambda 6548,6583$ doublets. Furthermore, we assumed that the lines are kinematically coupled having the same redshift and line width. This may not be true in all cases, because distinct ionised gas clouds with different kinematics and ionisation states can be aligned along our line-of-sight. The seeing may enhance this apparent confusion and in a few cases the redshift of the extended Balmer and [O III] lines do not agree in some regions of the host galaxy.
Measured line fluxes, line widths and the rest-frame velocity offset with respect to the redshift of the narrow QSO [O III] emission line are reported in Table 7. Statistical errors on the flux measurements and $3 \sigma$ upper limits for undetected emission lines were obtained based on the noise in the adjacent continuum.

On two EELR objects we have to comment in more detail: The $\mathrm{H} \beta$ line flux for the region A and B of PG $1612+261$ is unreliable due to a bad column on the PMAS CCD, but regions $\mathrm{C}$ and $\mathrm{D}$ remain unaffected. Off-nuclear long-slit spectra presented already by Boroson et al. (1982) showed that the [O III] $/ \mathrm{H} \beta$ line ratio is $\sim 10$ out to several arcseconds away from the nucleus. Thus, we assume that the gas is at least as highly ionised closer to the nucleus, although we were not able to reliably constrain the line ratios in that region. Furthermore, we find that the [O III] emission line in region $\mathrm{A}$ is significantly asymmetric requiring two independent Gaussian components to model the line profile. The QSO 3C 273 actually appears to be surrounded by some ionised gas, but it is by far the brightest object in our sample and was observed under poor seeing conditions. To check whether this feature may simply be an artefact of our data reduction or analysis we analysed the 3 individual exposures obtained for this target separately. The apparent structure could only be recovered in 1 of the 3 exposures implying that this may not be a real detection. Until observation with higher quality exists for 3C 273 we will treat it as a non-detection throughout the rest of this article.

No extended line emission was detected around the remaining 11 objects in the sample. We present their narrow-band images and available broad-band images in Fig. 8 for completeness.

\subsection{Comparison with HST narrow-band imaging}

Three QSOs among our sample are within the sample of the HST narrow-band snapshot survey by Bennert et al. (2002). This enables us to directly compare the narrow-band images obtained from both telescope (Fig. 9). We applied a median smoothing filter with a box size of $5 \times 5$ pixels to the HST images in order to catch lower surface brightness features. The $3 \sigma$ surface brightness limits for the HST and PMAS narrow-band images are listed in Table 6 . The estimated surface brightness limits support our visual impression from the comparison of the narrow-band images that the PMAS images are at least a factor of 4 deeper than the HST images even after smoothing.

PG 0026+129 was observed with the Planetary Camera (PC) that has a sampling of 0.05 pixel $^{-1}$ instead of $00^{\prime} 1$ pixel $^{-1} \mathrm{com}-$ pared to Wide Field Channel (WFC). Thus, the PMAS image for this particular object is roughly 20 times deeper than the one of HST, which was most likely the reason why the extended [O III] emission is completely missed by HST. The lowest surface brightness filaments around the nucleus in the HST image may tentatively correspond to the high surface brightness structures in our PMAS images. It is obvious that Bennert et al. (2002) underestimated the size of the extended [O III] emission for this object at least by factor of 3 .

The HST images of PG $0052+251$ and Mrk 1014 obtained with the WFC are deeper so that high surface brightness structures of extended [O III] emission could be detected. Nevertheless, our ground-bases PMAS narrow-band images reach a lot deeper, in particular in the case of Mrk 1014 where diffuse emission is present between the high surface brightness structures. Similar IFU observation of Mrk 1014 obtained by Fu \& Stockton (2008) display the same [O III] light distribution as our PMAS observation suggesting that the structures are real. The low surface brightness regions south-east of PG $0052+251$ was missed by Bennert et al. (2002) and became visible only 
B. Husemann et al.: The properties of the extended warm ionised gas around low-redshift QSOs
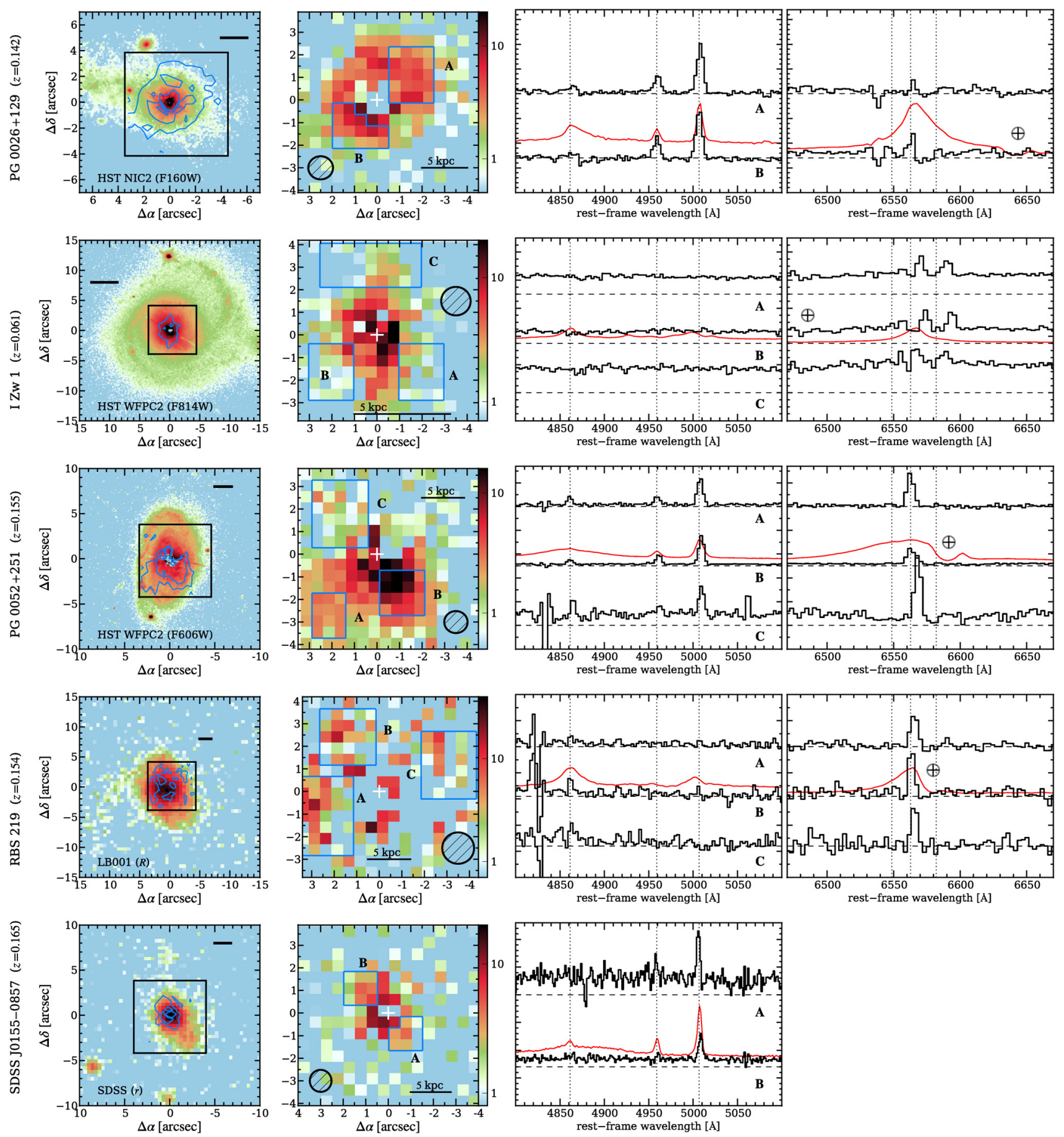

Fig. 7. Overview of the individual objects with extended emission after the point-like emission of the QSO was subtracted. Left panels: nucleussubtracted broad-band images with overplotted contours of extended line emission within the PMAS FoV (black box). A scale bar represents $5 \mathrm{kpc}$ at the object redshift and the image is oriented such that North is up and East is to the left. Middle panels: nucleus-subtracted [O III] narrow-band PMAS images with a logarithmic scaling in units of $10^{-16} \mathrm{erg} \mathrm{s}^{-1} \mathrm{~cm}^{-2} \operatorname{arcsec}^{-2}$. Note that in the case of I Zw 1, RBS 219 and PHL 1811, the $\mathrm{H} \alpha$ narrow-band images are shown instead. The blue boundaries indicate the spatial regions defined to obtain the co-added spectra shown in the right panels. A white cross marks the position of the QSO and the black ellipse indicates the FWHM of the PSF. Right panels: co-added spectra of the specific spatial regions around the $\mathrm{H} \beta$ and $\mathrm{H} \alpha$ line, if it was covered by the spectral range of the used instrument setup. The different spectra are arbitrarily scaled and offset from each other, for which the dashed lines indicate their corresponding zero flux density. Vertical dotted lines mark the position of emission lines at the QSO redshift, estimated from the peak of the narrow [O III] line or from the peak of the $\mathrm{H} \beta$ line when the [O III] line was too weak or exceptionally broad. The arbitrarily scaled QSO spectrum is shown as a red line for comparison. Telluric absorption line features are highlighted by a crossed circle symbol.

after smoothing the HST data. The maximum [O III] size was thus underestimated by a factor of $\sim 2$ in these two cases. We return to the issue of NLR sizes in Sect. 6.
For PG $0052+215$ we note further that the contours of the smoothed HST images do not agree with the [O III] light distribution in our PMAS images north of the nucleus. We carefully 

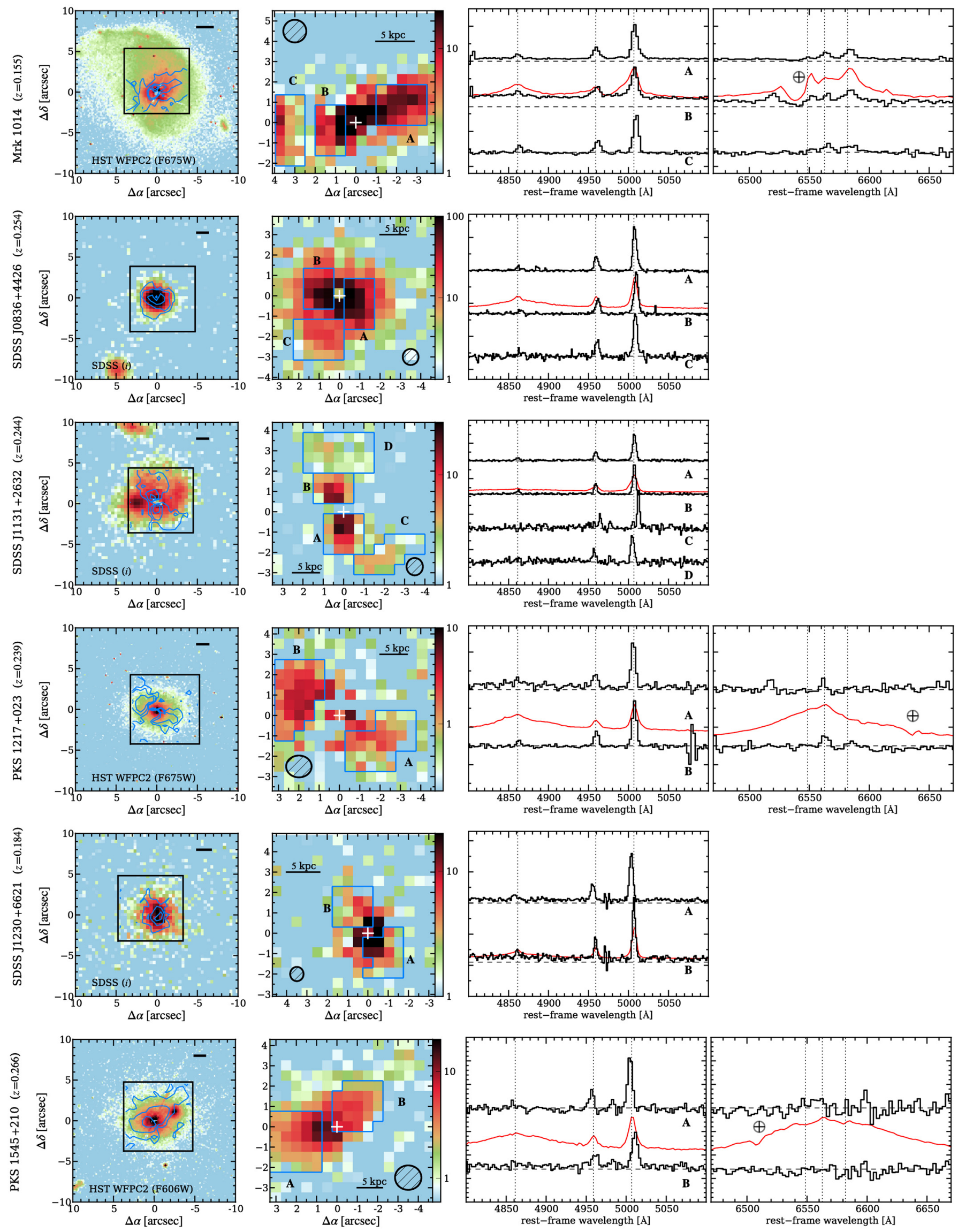

Fig. 7. continued. 
B. Husemann et al.: The properties of the extended warm ionised gas around low-redshift QSOs
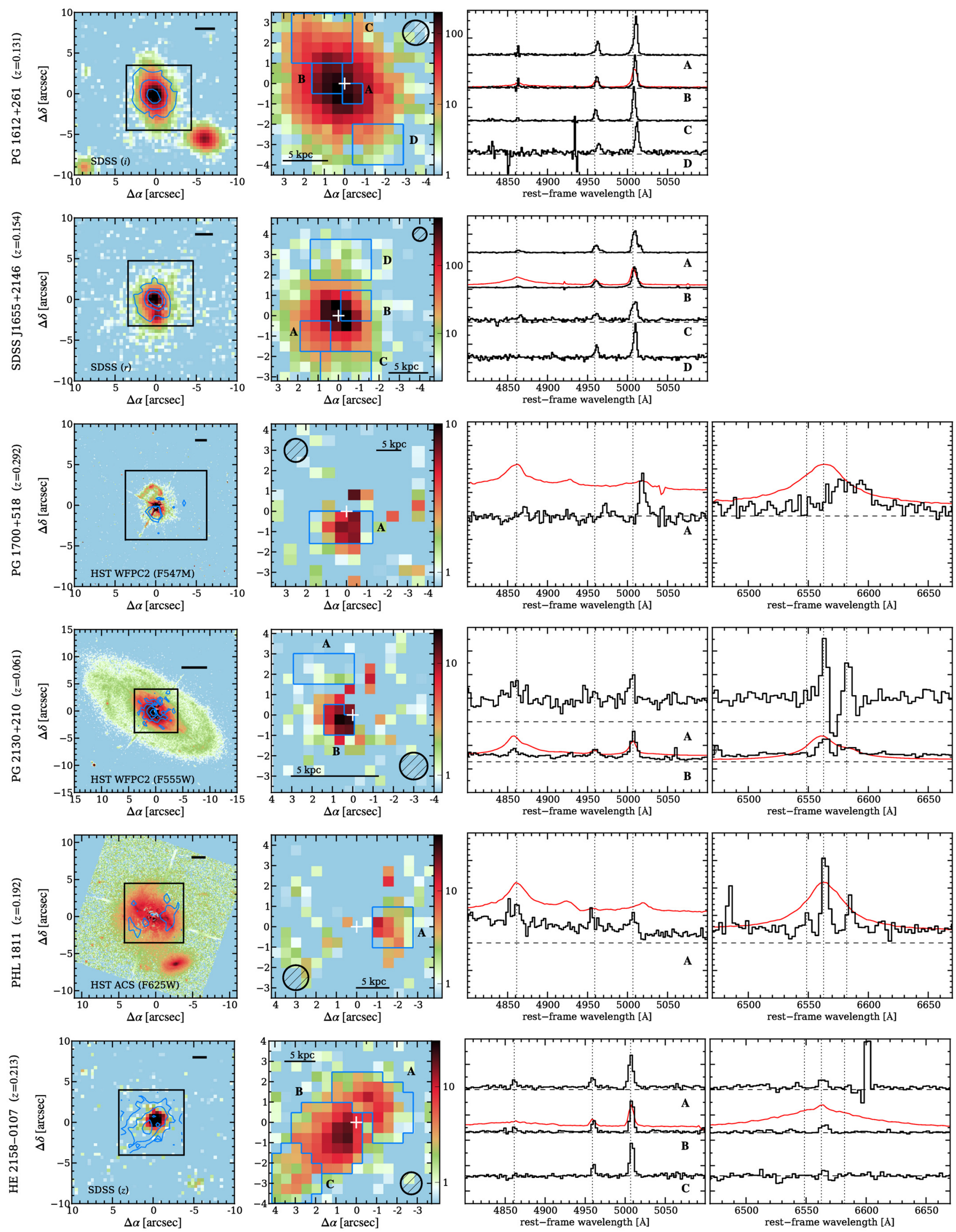

Fig. 7. continued. 

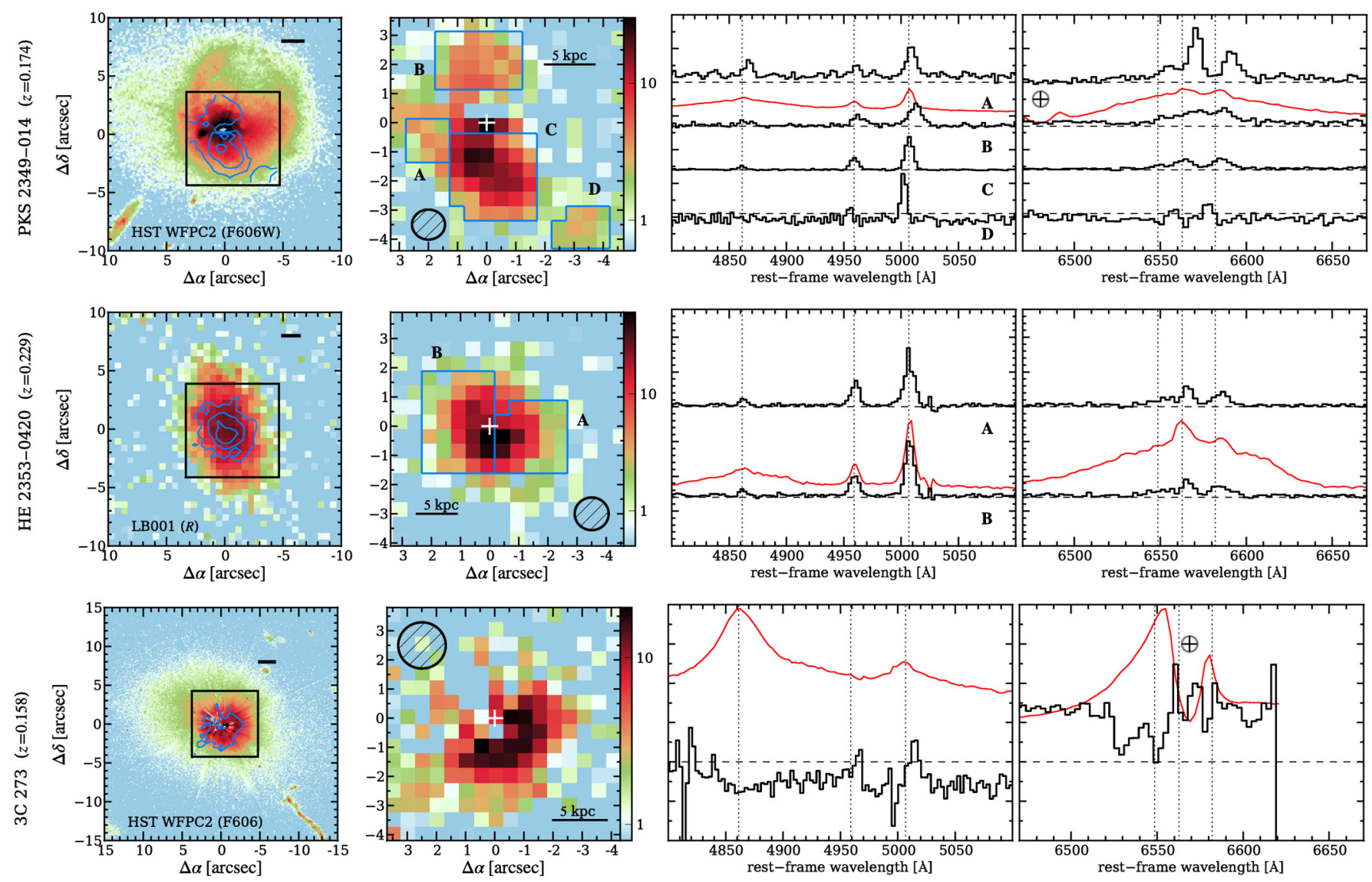

Fig. 7. continued.

checked that this is not introduced by our data reduction or data analysis. A possible origin for the rather symmetric diffuse light in the HST image around the nucleus might be continuum emission of the host. Bennert et al. (2002) subtracted only the contribution of the QSO using a PSF-star image, because the S/N in the much shallower continuum image of the host was insufficient. The unavailability of a continuum image also hampered the analysis of the narrow-band HST image of PKS 1545+210 (3C 323.1) as part of large HST snapshot program to study extended gas around radio galaxies (Privon et al. 2008).

We conclude that HST is of course much better in terms of spatial resolution than any optical ground-based observations, but the narrow-band images strongly suffer from its low sensitivity which has already been noted by several authors (e.g. Bennert 2005; Privon et al. 2008). Additionally, narrow-band images require off-band images which are often not sufficiently deep or absent to subtract the stellar continuum contribution from the on-band images.

\subsection{Ionisation sources of the extended ionised gas}

Narrow-band imaging observations of large AGN samples have been the main tool for detecting and characterising EELRs (e.g. Stockton \& MacKenty 1987; Baum et al. 1988; Mulchaey et al. 1996a; Bennert et al. 2002; Schmitt et al. 2003a,b; Privon et al. 2008). Most of these projects focused on a single emission line, which does not allow to constrain the origin of the gas ionisation. Three different ionisation mechanisms for the ISM on galactic scales need to be distinguished: 1 . photoionisation by the hard UV radiation field; 2 . shock ionisation due to winds or radio jets; 3. photoionisation due to the ionising UV photons of young and massive OB stars in star-forming region, i.e. classical $\mathrm{H}$ II regions. It is possible to distinguish between different excitation mechanisms with the aid of diagnostic emission-line ratio diagrams (e.g. Baldwin et al. 1981; Veilleux \& Osterbrock 1987; Villar-Martin et al. 1997; Allen et al. 1998). The most commonly used diagnostic diagram in the optical is the [O III] $\lambda 5007 / \mathrm{H} \beta$ vs. [N II] $\lambda 6583 / \mathrm{H} \alpha$ diagram (Veilleux \& Osterbrock 1987), also known as the BPT diagram (Baldwin et al. 1981). Those lines are usually the strongest in the optical spectrum and their ratios are most insensitive to reddening. Unfortunately, AGN photoionisation and shock-ionisation lead to very similar line ratios in the BPT diagram and cannot be reliable distinguished just from those lines. Anyway, both of these ionisation mechanisms are likely related to the AGN and we consider all corresponding line ratios being AGN ionised. AGN ionised and $\mathrm{H}$ II regions populate two distinct branches on this BPT diagram as shown in Fig. 10. A theoretical limit for the emission-line ratios of $\mathrm{H} \mathrm{II} \mathrm{re-}$ gions that can be reached during a maximum starburst event was derived by Kewley et al. (2001) as

$\log ([\mathrm{O} \mathrm{III}] / \mathrm{H} \beta)=0.61 /(\log ([\mathrm{N} \mathrm{II}] / \mathrm{H} \alpha)-0.47)+1.19$.

Based on a large sample of SDSS emission-line galaxies, Kauffmann et al. (2003) found that the AGN branch in the BPT diagram separates from $\mathrm{H}$ II branch well below the Kewley et al. demarcation curve and empirically defined a new line that tightly encloses the $\mathrm{H}$ II branch being parametrised as

$\log ([\mathrm{O} \mathrm{III}] / \mathrm{H} \beta)=0.61 /(\log ([\mathrm{N} \mathrm{II}] / \mathrm{H} \alpha)-0.05)+1.3$. 
B. Husemann et al.: The properties of the extended warm ionised gas around low-redshift QSOs
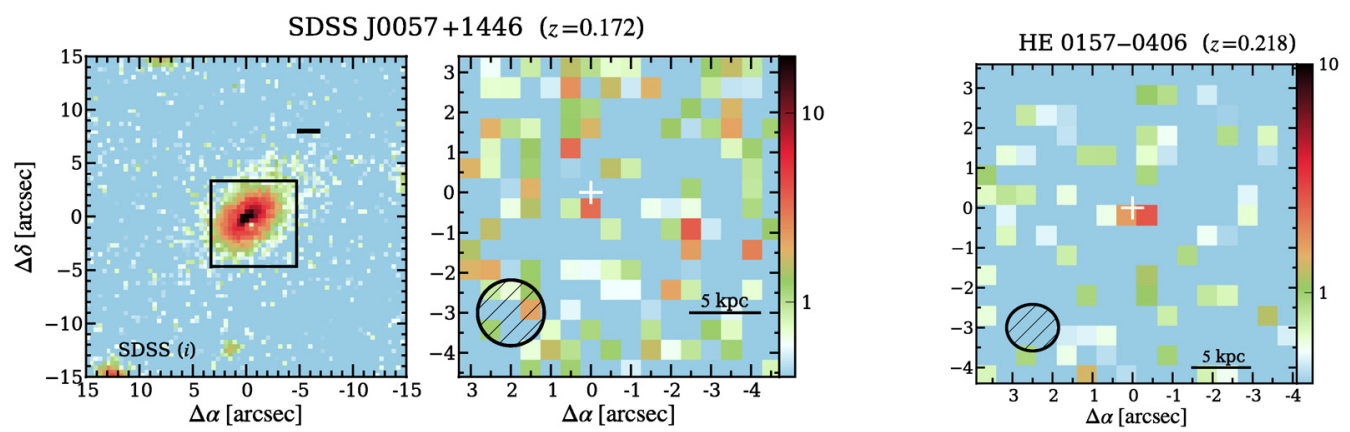

SDSS J0948 $+4335(z=0.172)$
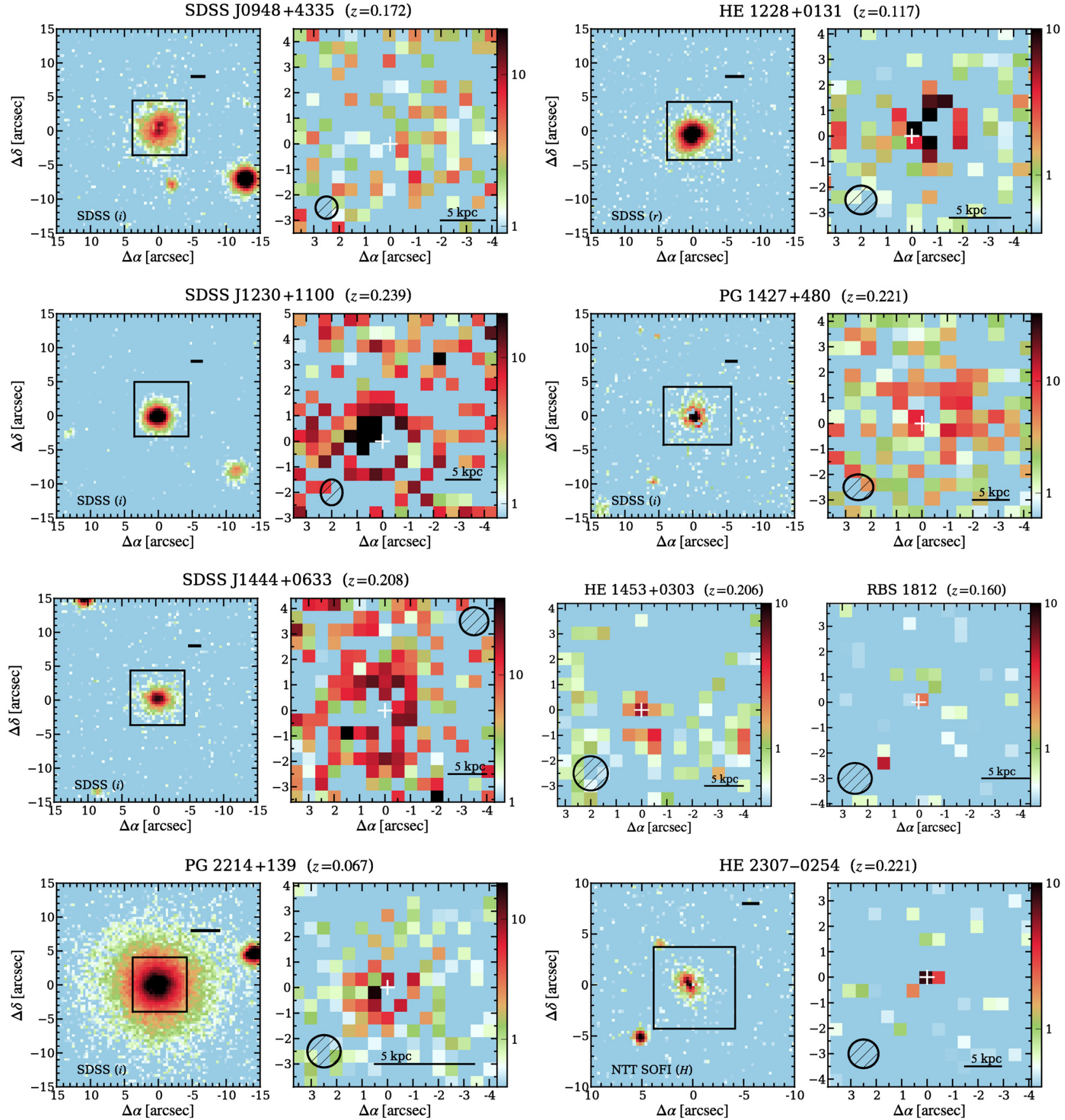

Fig. 8. Overview of the individual objects without detected extended emission. For each object the nucleus-subtracted broad-band images is presented, if available, in the left panel with the PMAS FoV indicated by the black rectangle. The black scale bar indicates a physical size of $5 \mathrm{kpc}$ at the redshift of the object. The nucleus-subtracted [O III] narrow-band images is presented in the right panels with the a white cross indicating the QSO position and the black ellipse indicates again the FWHM of the PSF. 

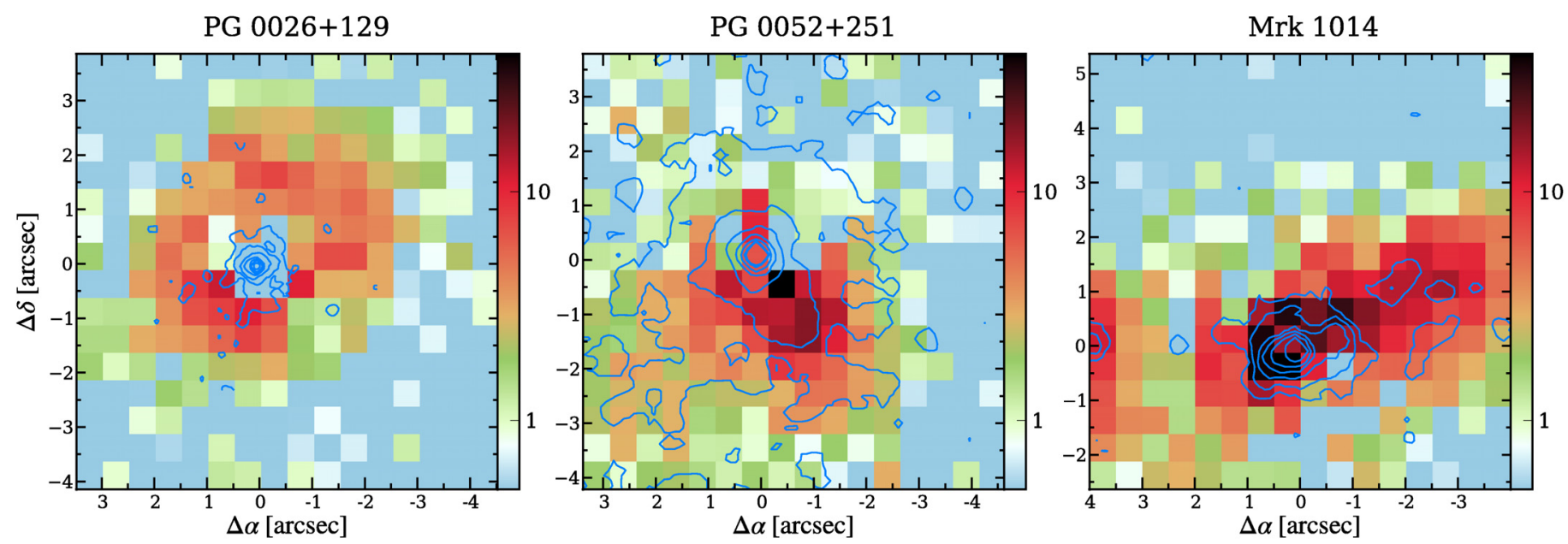

Fig. 9. Direct comparison of PMAS and HST [O III] narrow-band images. PMAS narrow-band images of PG 0026+129 (left panel), PG 0052+251 (middle panel) and Mrk 1014 (right panel) are shown in units of $10^{-16} \mathrm{erg} \mathrm{s}^{-1} \mathrm{~cm}^{-2} \operatorname{arcsec}^{-2}$. The corresponding HST narrow-band images of Bennert et al. (2002) are overplotted as blue contours also in a logarithmic scaling.

The region in between the two demarcation lines is often referred to as a composite region (Kauffmann et al. 2003; Kewley et al. 2006) where the emission-line gas could be partially ionised by both mechanisms. However, this classification approach may be oversimplified as pointed out by Cid Fernandes et al. (2010), because even emission-line ratios in the AGN area above the Kewley et al. curve may have some hidden contribution from H II region. Conversely, a region below that curve could be entirely powered by a massive starburst without requiring an AGN contribution. Furthermore, the AGN branch is composed of Seyfert type emission lines and low ionisation nuclear emission regions (LINERs, Heckman 1980) with lower [O III]/H $\beta$ at a given $[\mathrm{N} \mathrm{II}] / \mathrm{H} \alpha$ ratio. Stasińska et al. (2008) defined a demarcation line between the two classes at a angle of $59^{\circ}$, counter clock-wise from a line of constant $[\mathrm{O} \mathrm{III}] / \mathrm{H} \beta$, going through the point $(\log [\mathrm{N}$ II $] / \mathrm{H} \alpha=-0.43, \log [\mathrm{O}$ III $] / \mathrm{H} \beta=-0.49)$. As can be seen from Fig. 10, LINER-type line ratios are not found for any extended ionised gas region in our sample.

We created a BPT diagram (Fig. 10) for all the coadded spectra in which we could measure both line ratios, [O III $\lambda 5007 / \mathrm{H} \beta$ and $[\mathrm{N}$ II $] \lambda 6583 / \mathrm{H} \alpha$. In the case that either $\mathrm{H} \beta$ or $[\mathrm{N}$ II] $\lambda 6538$ was below the detection limit we used the $3 \sigma$ upper limit to constrain at least an upper/lower limit on the corresponding line ratio. Most of the emission-line ratios are on the AGN side above the Kewley et al. line for which we assume that AGN ionisation is the dominant process to ionise the gas. Region C of PG 0052+251, region A of PG 2130+099 and region A of PHL 1811 are almost directly on the H II branch and represent regions of ongoing star formation. In case of PHL 1811 the star forming region is coincident with spiral-like structure detected in the high-resolution HST image that was reported by Jenkins et al. (2005). Region A of PKS 2349-014 correspond to a physical companion located 4 " West of the nucleus and its emission-line ratios would place it in the composite region of the BPT diagram. However, the $\mathrm{H} \beta$ and $\mathrm{H} \alpha$ lines are redshifted by $\sim 200 \mathrm{~km} \mathrm{~s}^{-1}$ with respect to the [O III] lines. This can be explained most likely due to an seeing induced superposition of emission lines corresponding to an $\mathrm{H}$ II region and AGN ionised gas and we classify this particular emission-line region as solely being due to star formation. These knots of star formation are likely to found in interacting/merging systems, besides a large AGN ionised region, as reported by Villar-Martín et al. (2011b) from observations of type 2 QSO at redshift 0.3-0.6.

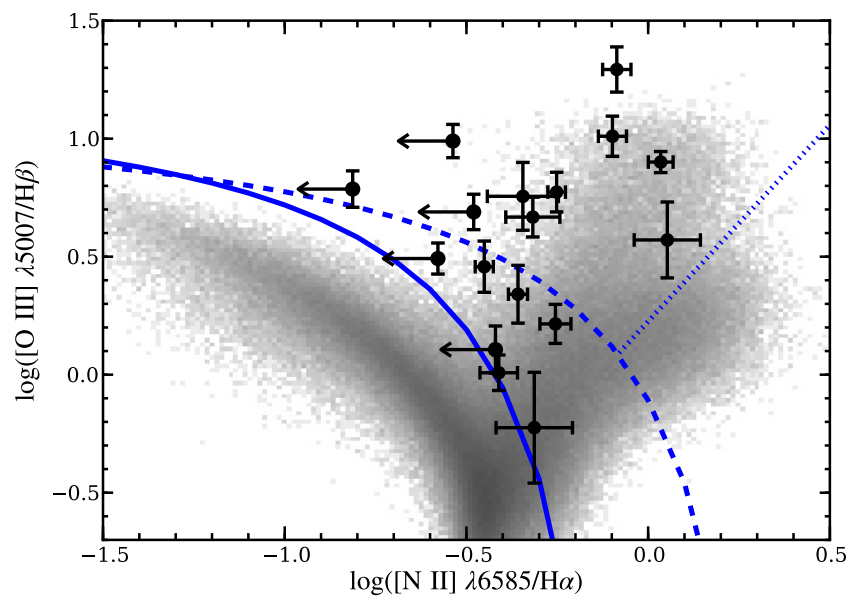

Fig. 10. The standard BPT $[\mathrm{O} \mathrm{III}] \lambda 5007 / \mathrm{H} \beta$ vs. [N II $] \lambda 6583 / \mathrm{H} \alpha$ diagram. The 2D histogram of the line ratios for more than 800000 emission-line galaxies taken from the SDSS DR7 value-added MPA/JHU galaxy catalogue are shown in the grey (logarithmic) scale image. All EELR regions of our sample for which both line ratios could be measured, even if one ratio is just a limit, are plotted as black symbols. Demarcation curves by Kewley et al. (2001), Kauffmann et al. (2003) and Stasińska et al. (2008) are drawn as solid, dashed and dotted blue lines, respectively.

For the majority of regions we are only able to infer a single emission line ratio, either [O III] $\lambda 5007 / \mathrm{H} \beta$ or [N II] $\lambda 6583 / \mathrm{H} \alpha$, so that the ionisation mechanism can generally not be determined unambiguously. We assumed that all regions with only an $\mathrm{H} \alpha$ detection are characteristic for $\mathrm{H}$ II regions. If these regions were instead ionised by AGN, the [O III] line would be at least as bright as the $\mathrm{H} \alpha$ line assuming a theoretical Balmer decrement of $\mathrm{H} \alpha / \mathrm{H} \beta \sim 2.87$ (Osterbrock \& Ferland 2006) and a $[\mathrm{O} \mathrm{III}] / \mathrm{H} \beta$ line ratio of $>3$ as a threshold for AGN ionised gas. Dust extinction along our line-of-sight within the host galaxy can dilute the $[\mathrm{O} \mathrm{III}]$ and $\mathrm{H} \beta$ emission further, but a high dust extinction is often found around H II regions and is directly proportional to the current star formation rate (SFR) (e.g. Hopkins et al. 2001; Sullivan et al. 2001). Thus AGN ionisation would be an unlikely scenario in those cases.

On the other hand, it is clear from the two branches in the BPT diagram that the $[\mathrm{O} \mathrm{III}] \lambda 5007 / \mathrm{H} \beta$ line ratio alone is not 
sufficient to constrain the ionisation mechanism. An emissionline ratio of $\log ([\mathrm{O} \mathrm{III}] \lambda 5007 / \mathrm{H} \beta)>0.9$ can still be securely assigned to AGN ionisation which is the case for 7 objects. Ho et al. (1997) defined a threshold value of [O III] $\lambda 5007 / \mathrm{H} \beta>3$ above which AGN ionisation is likely to be the dominant process. This can be physically explained by the fact that the star forming branch in the BPT diagram represents actually a sequence of increasing gas-phase metallicity from high to low $[\mathrm{O}$ III $] / \mathrm{H} \beta$ line ratios (e.g. Dopita et al. 2000; Pettini \& Pagel 2004). A high [O III] $\lambda 5007 / \mathrm{H} \beta$ line ratio can reasonably excluded as a signature for star formation for luminous AGN by considering the mass-metallicity relation of galaxies (e.g. Tremonti et al. 2004). Luminous AGN reside almost exclusively in massive galaxies above $M_{*}>10^{10} M_{\odot}$ (Kauffmann et al. 2003; Heckman et al. 2004), corresponding to a high gas-phase metallicity $\left(Z>Z_{\odot}\right)$. Thus, we will rely on the Ho et al. criterion to classify the ionisation of our EELRs, but we caution that the argument as outlined above might not be correct for all EELRs as the gas-phase metallicity in galaxies typically decreases with distance from the galaxy centre (e.g. Henry \& Worthey 1999).

From the detected regions of extended ionised gas we classify 13 of 19 to be entirely ionised by the AGN, 3 of 19 to be powered by $\mathrm{H}$ II regions only, and 3 of 19 exhibit signatures for both mechanisms dominating in spatially separated regions. Interestingly, all the $\mathrm{H}$ II regions are located in disc-dominated galaxies or in the strongly interacting system PKS 2349-014. Letawe et al. (2007) reported from their on-axis longslit spectroscopy of luminous QSOs that the ISM was in most cases ionised either by young stars or shocks. They found a completely AGN-ionised ISM only in the case of bulge-dominated galaxies, while their disc-dominated system or morphological disturbed galaxies show signatures of $\mathrm{H}$ II regions in almost every case. Since inactive disc-dominated galaxies are known to have young stellar population and continue to from star in their disc, it may not be surprising that this is true also for QSO host galaxies.

\subsection{Dependence on nuclear properties}

Compared to our earlier work presented in $\mathrm{Hu} 08$, we increased our sample by 11 objects and improved the QSO-host deblending algorithm. Our main result in $\mathrm{Hu} 08$ was that the presence of an EELR is closely correlated with certain spectral properties of the QSO itself, most prominently the EW of Fe II and the FWHM of the broad $\mathrm{H} \beta$ line. Both are the main drivers for the Eigenvector 1 parameter space (Boroson \& Green 1992) describing most of the variance among rest-frame optical QSO spectra. While this result is still valid, some of the new EELR detections require additional notes.

We classified I $Z_{w} 1$, RBS 219, PG 1700+518, PG 2130+099, and PHL 1811 as EELR non-detections in $\mathrm{Hu} 08$ for which we now find evidence for an EELR. All of them have a narrower $\mathrm{H} \beta$ width than the proposed threshold at $4000 \mathrm{~km} \mathrm{~s}^{-1}$ and significant Fe II emission in their spectra. The main point about the EELRs around I Zw 1, RBS 219 and PHL 1811 is that all the extended emission is ionised solely by star formation rather than AGN radiation. Thus, we refine our statement in $\mathrm{Hu} 08$ such that only the presence of extended AGN-ionised gas on kpc scales is correlated with the nuclear properties as described above. Our improved deblending technique allows us also to detect a small ENLR close to the nucleus of PG $1700+518$ and PG $2130+099$. Similar to Mrk 1014, which we noted as an outlier in Hu08, these objects are known to host a triple radio source. Radio jets may therefore be an additional ingredient altering the general trends, which we will explore more closely in the next sections.

The additional 11 SDSS QSOs were specifically selected from their nuclear properties as candidates to show luminous ENLRs. This could be confirmed in most cases. Of the 4 EELR non-detections, 3 were observed through clouds (cf. Table 4) which substantially reduced our chance to detect low surface brightness features. Thus, our statistics are not significantly increased compared to $\mathrm{Hu} 08$ and we focus on a detailed study of the ENLR ${ }^{4}$. The detected ENLRs in the remaining 7 new objects fit well into the correlations of $\mathrm{Hu} 08$, reinforcing the results reported in that paper.

\section{Extended narrow-line regions around QSOs}

\subsection{The ENLR morphology}

As a first step, we classified the ENLR morphologically by eye into three categories: "one-sided" (3 objects), "biconical" (6 objects) and "round" (7 objects). Assuming elliptical isophotes, we also measured the ellipticity $(1-b / a)$ of the ENLR from the light distribution at a level of $2 \sigma$ above the background noise in the reconstructed [O III] narrow-band images. Furthermore, we estimated the position angle (PA) of the major ENLR axis by searching for the maximum flux within bipolar cones of $20^{\circ}$ opening angle. All these parameters are summarised in Table 8. The ellipticities of the ENLRs agree with our visual impression of round and biconical morphologies with a transition value at $e \simeq 0.4$. An exception is PKS $2349-014$ because its ENLR is quite lopsided and the southern high surface brightness region dominates the whole ENLR structure.

We find a large fraction of $37.5 \%$ biconical ENLRs which even increases to $56 \%$ when the one-sided ENLRs are also assumed to be intrinsically biconical. The biconical fraction is in agreement with the one determined by Schmitt et al. (2003b) for type 1 Seyfert galaxies. However, it is expected that the opening angle of AGN ionisation cones increases with the luminosity of the AGN in the "receding torus" model (Lawrence 1991). Thus, biconical/elongated ENLRs should be less frequent than in lower luminosity Seyfert galaxies. This may indicate that the ENLR morphology is significantly affected by secondary properties, such as the gas density distribution with respect to the ionisation cones (Mulchaey et al. 1996b), or by the presence and direction of a radio jet.

A close alignment between the EELR and radio axis particularly around high-redshift RLQs and radio galaxies has been well established (McCarthy et al. 1987, 1995), which is often recognised also for lower luminosity Seyfert galaxies (Wilson \& Tsvetanov 1994; Capetti et al. 1996; Falcke et al. 1998; Nagar et al. 1999; Schmitt et al. 2003b) on much smaller physical scales. In Fig. 11 we directly compare the ENLR light distribution against the radio morphology for those QSO with available interferometric radio data. Among the 7 objects for which the PA of the radio jet is known, only PKS $1545+210$ is unaligned with respect to the radio axis, while the axes are aligned within $\pm 20^{\circ}$ (rms) for the majority of QSOs. This supports the view that radio jets even with relatively low power $\left(<3 \times 10^{24} \mathrm{~W} \mathrm{~Hz}^{-1}\right.$ at $1.4 \mathrm{GHz}$ ) can have a significant influence on the morphology and characteristics of the ENLR.

\footnotetext{
4 Of course an ENLR is also an EELR, but we want to emphasize the AGN-dominated ionisation source with a different naming following the literature. Thus, an ENLR may either be embedded in a larger EELR structure, or representing the entire EELR.
} 

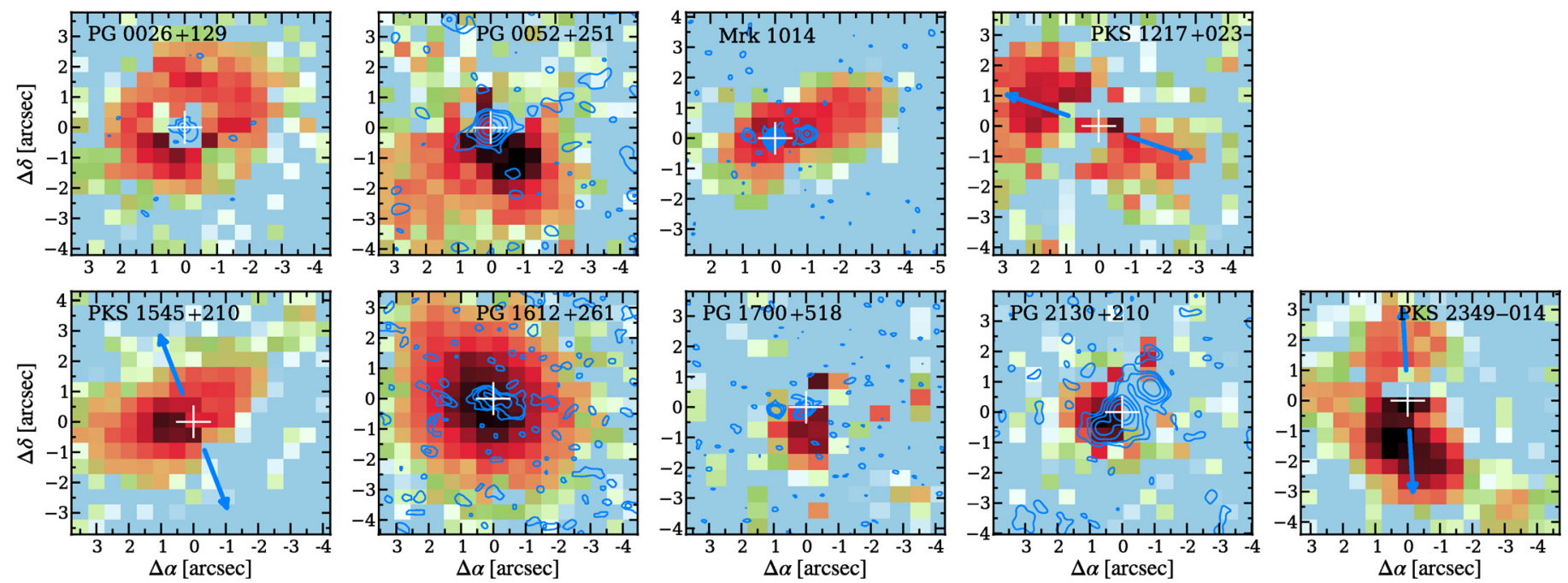

Fig. 11. The nucleus-subtracted ENLR light distribution of the [O III] line in comparison to the resolved radio emission. For the RQQs the high spatial resolution VLA radio images are overplotted as blue contours, while the primary radio axis of the radio lobes in RLQs is indicated by the blue arrows. The white cross marks the position of the QSO on which the radio emission is centred.

Table 8. Luminosity and morphological parameters of the ENLR.

\begin{tabular}{|c|c|c|c|c|}
\hline Name & $\begin{array}{c}\log L_{\mathrm{ENLR}}([\mathrm{O} \mathrm{III}]) \\
{\left[\mathrm{erg} \mathrm{s}^{-1}\right]}\end{array}$ & Morph. & $e$ & $\begin{array}{l}\text { PA } \\
{\left[{ }^{\circ}\right]}\end{array}$ \\
\hline \multicolumn{5}{|c|}{ detected ENLRs } \\
\hline PG $0026+129$ & $41.80 \pm 0.14$ & round & 0.30 & -57 \\
\hline PG $0052+251$ & $41.75 \pm 0.13$ & one-sided & . & 34 \\
\hline SDSS J0155-0857 & $41.43 \pm 0.19$ & round & 0.39 & 5 \\
\hline Mrk 1014 & $42.22 \pm 0.14$ & biconical & 0.60 & -65 \\
\hline SDSS J0846+4426 & $43.08 \pm 0.09$ & round & 0.09 & 83 \\
\hline SDSS J1131+2623 & $42.11 \pm 0.14$ & biconical & 0.64 & 18 \\
\hline PKS 1217+023 & $41.82 \pm 0.16$ & biconical & 0.44 & 62 \\
\hline SDSS J1230+6621 & $41.90 \pm 0.15$ & round & 0.32 & 5 \\
\hline PKS 1545+210 & $42.35 \pm 0.13$ & biconical & 0.45 & -49 \\
\hline PG 1612+261 & $42.80 \pm 0.12$ & round & 0.20 & 39 \\
\hline SDSS J1655+2146 & $43.03 \pm 0.09$ & round & 0.19 & 43 \\
\hline PG $1700+518$ & $41.81 \pm 0.18$ & one-sided & $\ldots$ & -27 \\
\hline PG 2130+099 & $40.90 \pm 0.32$ & one-sided & $\ldots$ & -28 \\
\hline HE 2158-0107 & $42.32 \pm 0.13$ & biconical & 0.46 & -46 \\
\hline PKS 2349-014 & $42.11 \pm 0.11$ & biconical & 0.18 & 6 \\
\hline HE 2353-0420 & $42.47 \pm 0.12$ & round & 0.15 & 57 \\
\hline \multicolumn{5}{|c|}{ undetected ENLRs } \\
\hline I Zw 1 & $<41.07$ & $\ldots$ & $\ldots$ & $\ldots$ \\
\hline SDSS J0057+1446 & $<41.90$ & $\ldots$ & $\ldots$ & $\ldots$ \\
\hline RBS 219 & $<41.57$ & $\ldots$ & $\ldots$ & $\ldots$ \\
\hline HE 0157-0406 & $<41.84$ & $\ldots$ & $\ldots$ & $\ldots$ \\
\hline SDSS J0948+4335 & $<42.66$ & $\ldots$ & $\ldots$ & $\ldots$ \\
\hline $3 \mathrm{C} 273$ & $<42.10$ & $\ldots$ & $\ldots$ & $\ldots$ \\
\hline HE $1228+0131$ & $<41.51$ & $\ldots$ & $\ldots$ & $\ldots$ \\
\hline SDSS J1230+1100 & $<42.86$ & $\ldots$ & $\ldots$ & $\ldots$ \\
\hline PG $1427+480$ & $<42.42$ & $\ldots$ & $\ldots$ & $\ldots$ \\
\hline SDSS J1444+0633 & $<42.97$ & $\ldots$ & $\ldots$ & $\ldots$ \\
\hline HE 1453-0303 & $<41.77$ & $\ldots$ & $\ldots$ & $\ldots$ \\
\hline PHL 1811 & $<41.72$ & $\ldots$ & $\ldots$ & $\ldots$ \\
\hline RBS 1812 & $<41.51$ & $\ldots$ & $\ldots$ & $\ldots$ \\
\hline HE 2307-0254 & $<41.63$ & $\ldots$ & $\ldots$ & $\ldots$ \\
\hline PG 2214+139 & $<41.03$ & $\ldots$ & $\ldots$ & $\ldots$ \\
\hline
\end{tabular}

\subsection{ENLR size measurements}

Previous ENLR surveys used various definitions for the ENLR size. Ground-based narrow-band images of 47 Seyfert galaxies were analysed by Mulchaey et al. (1996a), who measured isophotal ENLR sizes at a limiting surface brightness of $2 \times 10^{-16} \mathrm{erg} \mathrm{s}^{-1} \mathrm{~cm}^{-2} \operatorname{arcsec}^{-2}$. Circular annuli were used by Bennert et al. (2002) to infer the ENLR radii at which the [O III] surface brightness dropped below the background surface brightness limit. Finally, Schmitt et al. (2003a) estimated the maximum projected sizes of the [O III] light distribution by eye from their HST narrow-band images. Thus, different ENLR size definitions are not directly comparable with each other, and some of them depend critically on the depth of the data.

We measured three different ENLR sizes from the [O III] light distribution at $2 \sigma$ above the background noise: 1) the $r_{95}$ radius defined as the circular aperture containing $95 \%$ of the ENLR [O III] flux, 2) the effective radius $r_{\mathrm{e}}$, defined as the luminosity-weighted mean of projected pixel distances to the centre, and 3) the maximum isophotal radius $r_{\text {iso }}$ out to a limiting [O III] surface brightness of $2 \times 10^{-16} \mathrm{erg} \mathrm{s}^{-1} \mathrm{~cm}^{-2} \operatorname{arcsec}^{-2}$. The measured ENLR sizes are reported in Table 9 with errors based on Monte Carlo simulations of the QSO-host deblending process. We generated 200 different realisations of each object datacube consistent with the variance in each pixel, which we similarly analysed to infer $1 \sigma$ errors as the rms of the parameters. Because of the low spatial sampling of our reconstructed PMAS narrow-band images, we further added 0.'5 to the uncertainty of $r_{95}$, and $r_{\text {iso }}$ as well as 0.25 to the uncertainty of $r_{\mathrm{e}}$. The seeing certainly increases the apparent size of the ENLR, but its strength strongly depends on the intrinsic emission line distribution and also on the size definition. We emphasise that any correction for the seeing would be a small effect given the large apparent ENLR sizes of several arcseconds, so that we did not perform any seeing correction. The ENLR apparently reaches beyond the PMAS FoV in three cases, HE 2158-0107, Mrk 1014, and PG 1612+261, for which we set lower limits to their ENLR sizes. For all other objects the surface brightness in the ENLR is sufficiently low at the edge of the PMAS FoV so that no significant extension of the ENLR is expected beyond the FoV.

Because of the high frequency of elongated ENLR structures, we tested by how much longslit spectroscopic observations would underestimate the ENLR size if not properly aligned along the ENLR major axis. We extracted synthetic longslit surface brightness distributions from the narrow-band images along 
Table 9. Measured sizes of the ENLR.

\begin{tabular}{lccc}
\hline \hline Name & $\begin{array}{c}r_{95} \\
{\left[{ }^{\prime}\right]}\end{array}$ & $\begin{array}{c}r_{\mathrm{e}} \\
{\left[{ }^{\prime \prime}\right]}\end{array}$ & $\begin{array}{c}r_{\text {iso }} \\
{\left[{ }^{\prime \prime}\right]}\end{array}$ \\
\hline PG 0026+129 & $3.4 \pm 0.7$ & $1.9 \pm 0.3$ & $3.1 \pm 0.8$ \\
PG 0052+251 & $3.4 \pm 0.6$ & $1.7 \pm 0.4$ & $4.1 \pm 0.8$ \\
SDSS J0155-0857 & $2.0 \pm 1.3$ & $1.0 \pm 0.4$ & $2.0 \pm 1.5$ \\
Mrk 1014 & $>3.9$ & $>1.9$ & $>4.3$ \\
SDSS J0846+4426 & $2.6 \pm 1.1$ & $1.3 \pm 0.4$ & $3.1 \pm 1.4$ \\
SDSS J1131+2623 & $3.3 \pm 0.8$ & $1.4 \pm 0.4$ & $4.5 \pm 1.0$ \\
PKS 1217+023 & $3.3 \pm 0.6$ & $2.1 \pm 0.4$ & $3.2 \pm 0.7$ \\
SDSS J1230+6621 & $1.6 \pm 0.9$ & $0.8 \pm 0.4$ & $2.0 \pm 0.8$ \\
PKS 1545+210 & $3.4 \pm 0.7$ & $1.8 \pm 0.4$ & $3.2 \pm 0.7$ \\
PG 1612+261 & $>2.7$ & $>1.3$ & $>3.3$ \\
SDSS J1655+2146 & $2.3 \pm 0.6$ & $1.0 \pm 0.3$ & $3.1 \pm 0.7$ \\
PG 1700+518 & $3.6 \pm 1.0$ & $1.8 \pm 0.4$ & $2.2 \pm 1.8$ \\
PG 2130+099 & $2.5 \pm 1.1$ & $1.3 \pm 0.5$ & $1.8 \pm 1.4$ \\
HE 2158-0107 & $>3.4$ & $>1.9$ & $>4.8$ \\
PKS 2349-014 & $3.1 \pm 0.6$ & $1.8 \pm 0.3$ & $5.1 \pm 1.2$ \\
HE 2353-0420 & $2.5 \pm 0.6$ & $1.1 \pm 0.3$ & $2.3 \pm 0.6$ \\
\hline
\end{tabular}

the major and minor axis of the ENLR. We find that ENLR sizes are underestimated on average by a factor of 2 when the slit is oriented along the major axis of the ENLR. For individual objects even a factor of 6 can be reached. Thus, the choice of the longslit position angle has a stronger impact on the inferred ENLR size than the $30 \%$ reported by Greene et al. (2011) based on their multiple longslit observations of type 2 QSOs. One possible explanation for this discrepancy could be the confusion of extended and unresolved [O III] emission of the nucleus, enhanced by the seeing. We can deblend these two component in our QSO using the broad emission lines, but those are not visible in the spectra of type 2 QSOs by definition.

\subsection{ENLR size-luminosity relation}

In Fig. 12 we compare the projected physical ENLR size against the total [O III] luminosity (ENLR+QSO) (left panels) and the bolometric luminosity $L_{\text {bol }}$ of the QSO (right panels). The ENLR size appears to be almost uncorrelated with the [O III] luminosity given the low Spearman rank correlation coefficient and a high probability for no correlation ( $\rho=0.27, P=0.3$, for $\left.r_{95}\right)$. We find that the correlation coefficient is significantly higher for the QSO continuum luminosity $L_{5100}\left(\rho=0.65, P=6 \times 10^{-3}\right.$, for $r_{95}$ ). The correlation between the ENLR size and the QSO continuum luminosity has not been investigated so far, because all previous studies focused either on type 2 AGN or on lowluminosity Seyfert nuclei which often exhibit a substantial contamination by the host galaxy continuum. Bennert et al. (2002) compared the ENLR size also with the broad $\mathrm{H} \beta$ luminosity of the QSOs which is well correlated with the QSO continuum luminosity (e.g. Greene \& Ho 2005; Schulze et al. 2009; Punsly $\&$ Zhang 2011). Their data also imply a higher correlation coefficient for the ENLR size as a function of $\mathrm{H} \beta$ luminosity which however they did not notice in the paper. The fact that ENLR size is much better correlated with the AGN continuum luminosity than with the normally used [O III] luminosity shows that the [O III] luminosity is a less reliable proxy for the amount of ionising photons compared to the continuum luminosity, although the [O III] luminosity has been commonly found to scale well with the QSO luminosity (e.g. Zakamska et al. 2003; Heckman et al. 2004).

Because reliable ENLR characterisation are scarce for lower luminosity Seyfert 1 galaxies, we cannot study the ENLR size-luminosity relation in detail in this paper and restrict ourselves to average quantities. The mean size of the detected ENLRs for our type 1 QSOs is $r_{\mathrm{e}}=5 \mathrm{kpc} \pm 2 \mathrm{kpc}$ and $r_{95}=$ $10 \mathrm{kpc} \pm 3 \mathrm{kpc}$ with a similar value for $r_{\text {iso }}$ at a median [O III] luminosity of $\log \left(L([\mathrm{O} \mathrm{III}]) /\left[\mathrm{erg} \mathrm{s}^{-1}\right]\right)=42.7 \pm 0.15$. Just a few objects seem to follow the scaling relation of Bennert et al. (2002), while the majority are a factor of 2 larger than the extrapolation of their relation to the corresponding [O III] luminosities. The discrepancy is even larger for the scaling relation of Schmitt et al. (2003b) for their Seyfert 1 sub-sample. This is may be unsurprising given the fact that the limiting surface brightness of the HST narrow-band images is at least a factor of 10 higher. The samples of Greene et al. (2011) and Fu et al. (2012) have [O III] luminosities and limiting surface brightnesses $\left(\sim 1 \times 10^{-16} \mathrm{erg} \mathrm{s}^{-1} \mathrm{~cm}^{-2} \operatorname{arcsec}^{-2}\right)$ comparable to our sample of type 1 QSOs. Their reported ENLR sizes are of the order of $10 \mathrm{kpc}$, which matches with the results from our sample, although their ENLR sizes seem a bit larger at lower [O III] luminosities (cf. Fig. 12). However, when considering the continuum luminosities for the few type 1 QSOs within the sample of $\mathrm{Fu}$ et al. this discrepancy is largely reduced.

The combined data of all studies strongly suggests that the ENLR scaling relation cannot be as different for type 1 and type 2 AGN as implied by the results of Schmitt et al. (2003b). If we extrapolate their relation to an [O III] luminosity $\log \left(L([\mathrm{O} \mathrm{III}]) /\left[\mathrm{erg} \mathrm{s}^{-1}\right]\right)=42.5$, it would imply an ENLR size at least a factor of 2 lower for type 2 QSOs than for type 1 QSOs. Additionally, it is obvious that the zero-point of the ENLR sizeluminosity relation based on HST data is too low because of the surface brightness sensitivity as discussed above. The slope of the relation is a different issue and may be a physically more interesting parameter, but cannot be inferred from our small range in luminosities. Greene et al. (2011) determined a shallow ENLR size-luminosity relation only because ENLR sizes from Seyfert 2 galaxies were taken into account. Those were estimated by extrapolation of the NLR surface brightness profiles to large radii based on longslit spectroscopy (Fraquelli et al. 2003). Whether this is a robust procedure to obtain reliable and unbiased (E)NLR sizes for low-luminosity AGN still needs to be confirmed by direct observations.

Netzer et al. (2004) emphasised that the ENLR sizeluminosity relation should not be a universal law. The ENLR size of luminous QSOs at high redshift, implied by the current relation, would exceed those of the host galaxies by more than an order of magnitude. Our QSOs are just at the limit where the ISM of the entire host galaxies is ionised. so that we do not yet see a break in the ENLR size-luminosity relation. On the other hand, we were unable to detect any ENLR for about half of our QSO sample, at similar QSO continuum luminosities. Thus, it is not clear whether a universal ENLR size-luminosity relation does actually exist. A few of our ENLR non-detections are associated with compact and apparently undisturbed host galaxies, such as PG 1427+480 (cf. Fig. 7), which might have a comparably small ENLR size that cannot be resolved by our observations. It is possible that the host galaxy size imposes a natural limit for the ENLR because of the sharp decline in gas density. Alternatively, galaxy interactions/mergers might increase the amount of available gas illuminated by the QSO in contrast to objects with nondetected ENLRs as suggested by Matsuoka (2012), which would enhance the visibility of the ENLR and possibly also its size. We already emphasized in $\mathrm{Hu} 08$ that the detection or non-detection of an ENLR correlates with certain spectral properties of the QSO that drive the variance along the Eigenvector 1 parameter space (Boroson \& Green 1992). It is puzzling how the EW 

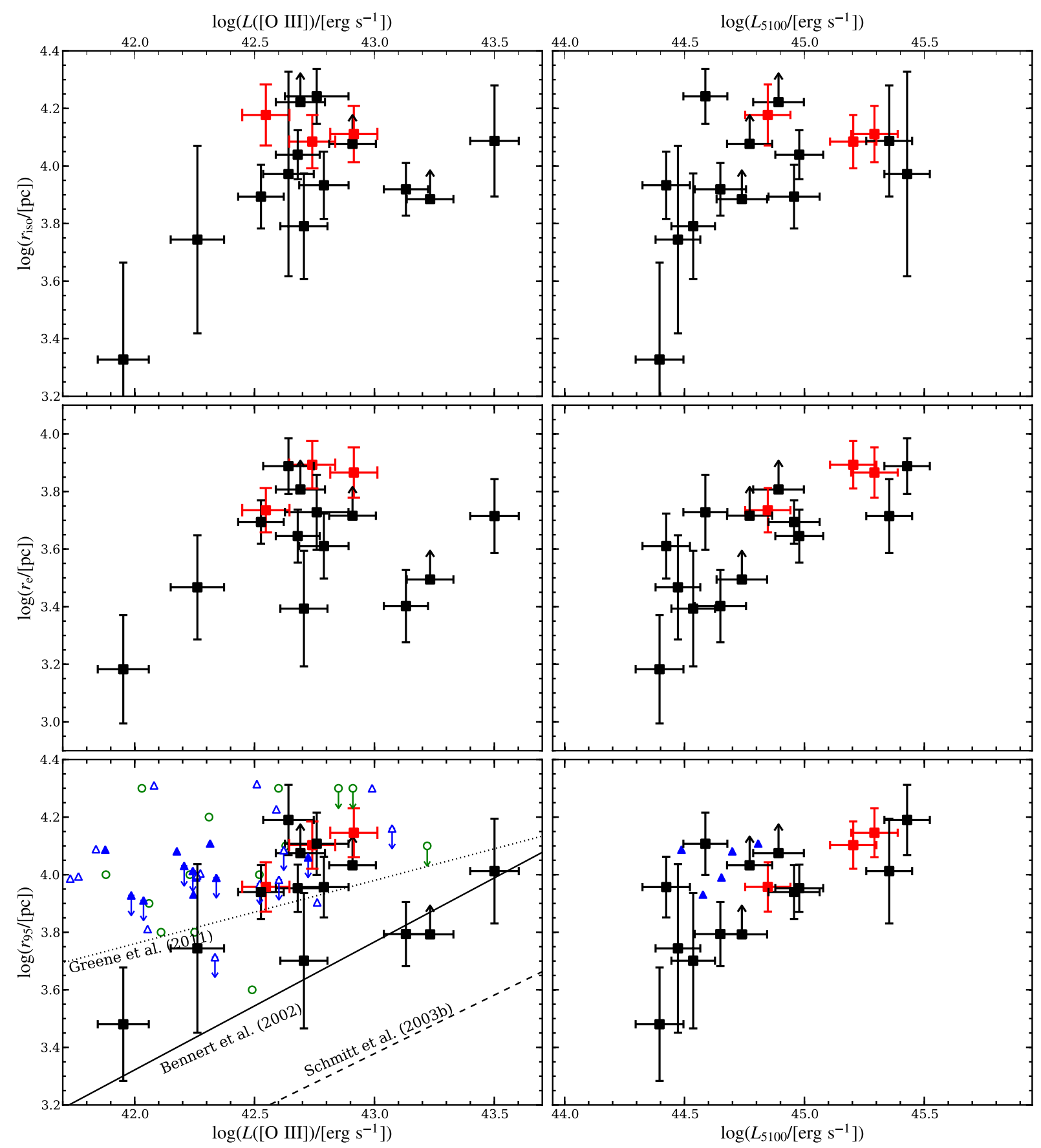

Fig. 12. ENLR sizes as a function of total [O III] luminosity (left panels) and the QSO continuum luminosity at $5100 \AA$ A (right panels). The used size definitions are isophotal radius $r_{\text {iso }}$ (upper panels), effective radius $r_{\mathrm{e}}$ (middle panels), and the radius containing 95\% of the ENLR flux $r_{95}$ (lower panels). The solid, dashed and dotted lines in the lower left panel correspond to the ENLR size-luminosity relations of Bennert et al. (2002), Schmitt et al. (2003b), and Greene et al. (2011), respectively. The black filled symbols represent RQQs whereas the red filled symbols highlight the few RLQs in our sample. The green opened circles are measurement for luminous type 2 QSOs as reported by Greene et al. (2011) and the blue triangles are measurements for double-peaked AGN (open symbols denote type 2 and filled type 1 AGN) provided by Fu et al. (2012).

Fe II in the nuclear spectrum can be causally connected with properties of the ENLR on kpc scales. We doubt that this can be ascribed solely by galaxy interactions; fundamental physical properties of the nucleus that are yet to be identified probably play an important role.

\subsection{ENLR luminosities}

In addition to the [O III] luminosities of the resolved ENLR we also obtained robust upper limits for $L_{\text {ENLR }}([\mathrm{O} I I I])$ in the undetected cases. We added ENLRs with the observed shapes to the original datacubes until the input luminosity reached the detection limit. We consider an ENLR to be detected at $3 \sigma$ significance when the fluxes in 9 adjacent spaxel were above the $2 \sigma$ background noise level in $95 \%$ of 200 different Monte Carlo realisations. Here we implicitly assume that the ENLR luminosity is the limiting factor to detect an ENLR and not its size. The [O III] luminosity of the ENLRs $\left(L_{\mathrm{ENLR}}([\mathrm{O} \mathrm{III}])\right)$ is reported for all QSOs in Table 8 and compared with the unresolved [O III] luminosity of the nucleus $\left(L_{\mathrm{QSO}}([\mathrm{O} \mathrm{III}])\right)$ in Fig. 13. Our measurements indicate a correlation of the two different luminosities that is in agreement with the derived upper limits. With 


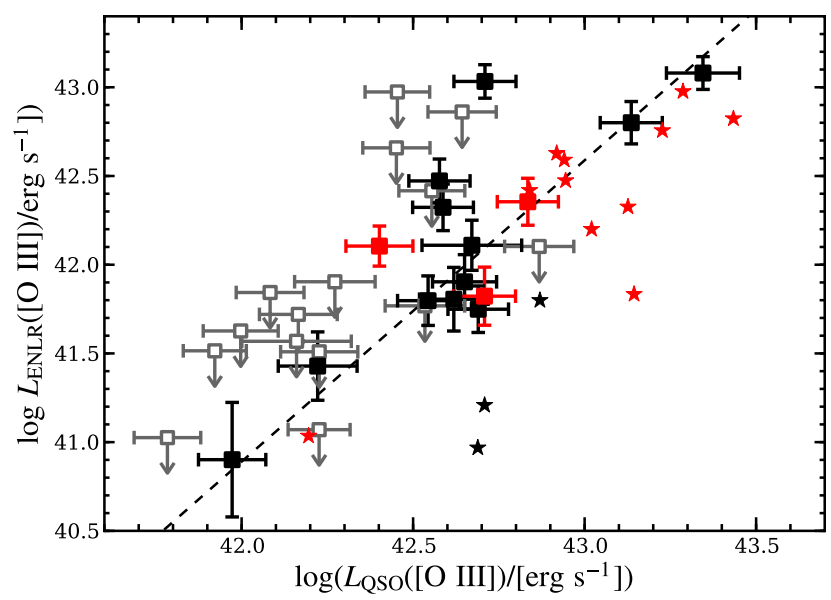

Fig. 13. [O III] luminosity of the ENLR as a function of the unresolved QSO [O III] luminosity. Squared symbols indicate our own PMAS measurements and star symbols refer to the literature data of Stockton \& MacKenty (1987). Radio-quiet and radio-loud objects are marked as black and red symbols, respectively. The open grey symbol represent upper limits and the dashed line is a linear fit to our PMAS data (squared symbols) only.

the Astronomy Survival Analysis package (ASURV, Lavalley et al. 1992) we determine a generalised Spearman rank correlation coefficients of $\rho=0.76$, taking the censored data into account. The probability for a non-correlation is $P<10^{-5}$ for the combined data set, which covers more than 2 orders of magnitude in [O III] luminosity. This possibly suggests that the nebulae are the extensions of the unresolved NLRs in terms of the covering factor for the ionising radiation of the AGN. A linear regression analysis with the Buckley-James method as part of ASURV yields a relation of

$\log \left(L_{\mathrm{ENLR}}([\mathrm{O} \mathrm{III}])\right)=(1.7 \pm 0.3) \log \left(L_{\mathrm{QSO}}([\mathrm{O} \mathrm{III}])\right)-(30.4 \pm 2.2)$

A similar trend was already reported from the [O III] narrowband survey of low-redshift QSOs by Stockton \& MacKenty (1987) and the narrow-band survey of local Seyfert galaxies carried out by Mulchaey et al. (1996a). The detected EELRs in the Stockton \& MacKenty (1987) sample are mostly associated with RLQs and appear to follow a similar scaling relation as implied by our data at high [O III] luminosities. That their measurements are systematically lower than our relation can be easily explained by the fact that they excluded a central aperture with a radius of $\sim 3^{\prime \prime}$ to avoid contamination by the QSO light.

If the [O III] luminosity was in general an indicator for the amount of ionising photons emitted by the AGN, we would also expect a significant correlation of the ENLR luminosity with the QSO continuum luminosity $L_{5100}$. We already reported in $\mathrm{Hu} 08$ that this is not the case and present the corresponding diagram now for our extended sample in Fig. 14. The generalised Spearman rank correlation coefficient computed with ASURV is $\rho=0.108$ with a probability of $P=0.55$ to be completely uncorrelated. This is rather surprising because of the strong correlation between the ENLR size and the continuum luminosity as shown in Fig. 12.

To investigate the cause of this lack of correlation, Matsuoka (2012) compared the AGN continuum luminosity against the Eddington ratio using a large compilation of available QSO data including the one presented in this article. They quote to see a clear anti-correlation between the Eddington ratio and the ENLR

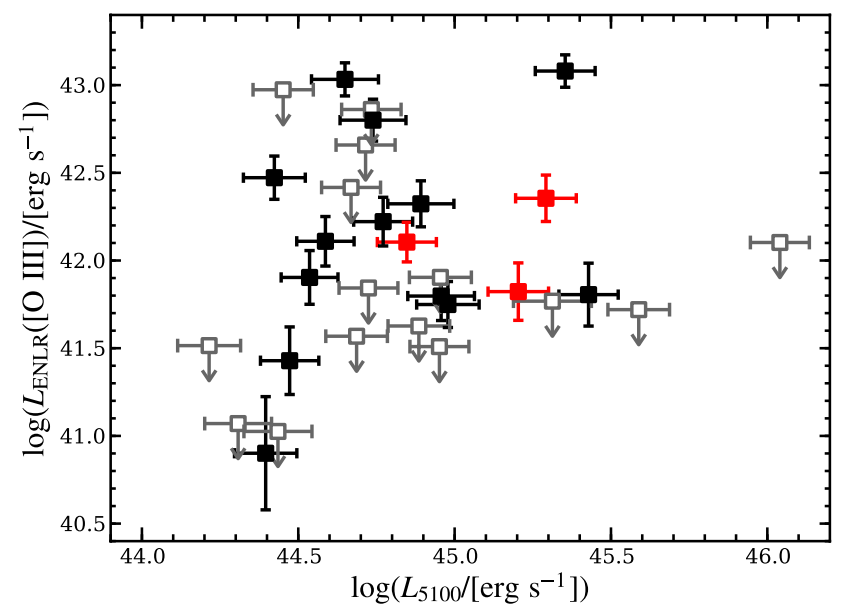

Fig. 14. [O III] luminosity of the ENLR as a function of QSO continuum luminosity at $5100 \AA$ A. Symbols are as defined in Fig. 13 .

detection rate and argue that this is likely caused by the availability of gas given the signs of interaction found in these systems (e.g. Husemann et al. 2010; Villar-Martín et al. 2011b). We show the distribution of our QSOs with ENLR detections and non-detection in the $M_{\mathrm{BH}}-L_{\mathrm{bol}}$ plane in Fig. 15. Many QSOs with a non-detected ENLR appear to populate a similar region than the ones with a detected ENLR. A two dimensional Kolmogorov-Smirnov (K-S) test (Peacock 1983; Fasano \& Franceschini 1987) confirms a high probability of $P=0.74$ that both samples (ENLR detections and non-detections) are drawn from the same parent population, which questions the trend proposed by Matsuoka (2012). What we can confirm is that we see a deficit of ENLR for QSOs close to Eddington ratio $(\log \lambda>-0.5)$, but the number of QSOs in our sample is too small in that regime to make a robust statements.

Nevertheless, the ENLR luminosity apparently cannot be determined solely by the amount of ionising photons, so that it seems indeed required to invoke the mass and distribution of gas in the host galaxy as additional parameters. Another aspect is the increasing evidence supporting the clumpy torus model (Nenkova et al. 2008; Mor et al. 2009; Alonso-Herrero et al. 2011). In this model it is just a matter of probability if our lineof-sight is intercept by dusty gas clouds of sufficient column density to obscure the nucleus. Thus, the intrinsic fraction of radiation that is able to escape into the host galaxy can have a large range even for apparent type 1 QSOs. It is therefore not surprising that the covering factor has been shown to be the dominant parameter that controls the [O III] luminosity in QSO spectra (Baskin \& Laor 2005). Interestingly, it was recently reported by $\mathrm{Xu}$ et al. (2012) that also the gas density of the ISM correlates with the nuclear properties in the sense that objects with narrow $\mathrm{H} \beta$ lines and stronger Fe II emission have a systematically denser ISM. This could imply that our non-detected ENLRs may be explained simply by the low efficiency of ionising photons to ionise the dense ISM clumps due to self-shielding, It may also even explain the presence of star formation, as high gas density clumps are more favourable to have cool molecular gas cores.

\subsection{Dependence on radio properties}

Radio observations have shown that the nuclear [O III] luminosity is correlated with the continuum radio luminosity in radio galaxies and RLQs (e.g. Baum \& Heckman 1989; Rawlings \& Saunders 1991; Xu et al. 1999) as well as in RQQs (e.g. Miller et al. 1993; Xu et al. 1999; Ho \& Ulvestad 2001). The radio 


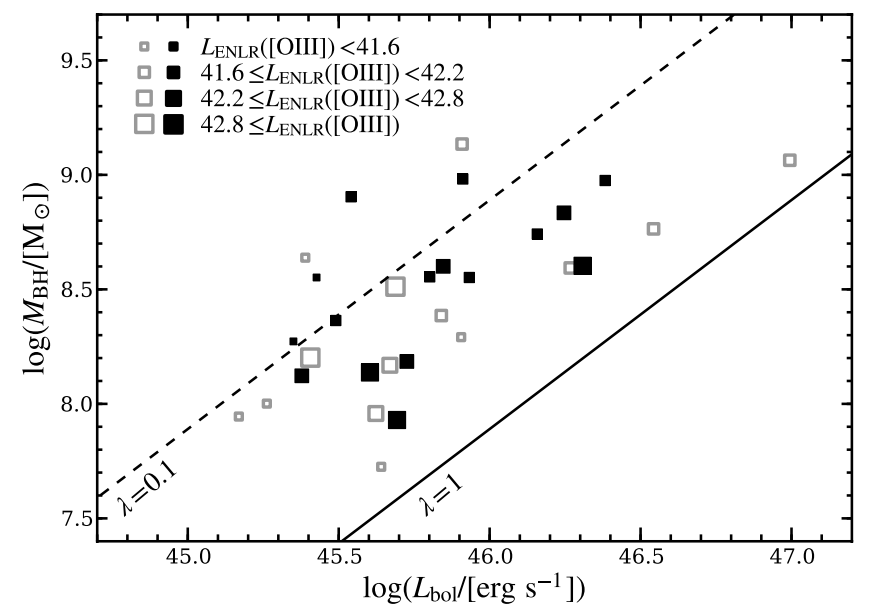

Fig. 15. Distribution of QSOs with detected and non-detected ENLRs in the $M_{\mathrm{BH}}-L_{\mathrm{bol}}$ plane. The ENLR luminosity or its upper limit is encoded in the symbol size. QSO with detected ENLR are indicated by filled symbols and non-detections by grey open symbols, respectively. Solid and dashed lines correspond to constant Eddington ratios of $\lambda=1$ and $\lambda=0.1$, respectively.

(6 cm) to optical (4400 $\AA$ ) flux ratio, the $R$ parameter, has often been used to distinguish between RQQs $(R<1)$ and RLQs $(R>10)$, so that their reported [O III] scaling relations often appear significantly offset from each other in terms of radio luminosity. Because the radio emission in RLQs is often dominated by their extended lobes, the two classes may follow a common relation only if the radio core power is considered ( $\mathrm{Xu}$ et al. 1999). Here we compare $L_{\text {ENLR }}([\mathrm{O} I I I])$ with the total radio luminosity at $1.4 \mathrm{GHz}$ (Fig. 16) excluding the few known RLQs in our sample. It is difficult to argue about a possible correlation between these two quantities because of the numerous upper limits in both axes, radio and ENLR luminosity. The generalised Spearman rank correlation coefficient is $\rho=0.56$ with $P=0.003$ for the non-correlation case. Nevertheless, it seems that the ENLR luminosity depends more strongly on the radio luminosity than on the QSO continuum luminosity, which we find is supported by three facts:

1. an ENLR is detected around all QSOs with $\log \left(L(1.4 \mathrm{GHz}) /\left[\mathrm{W} \mathrm{Hz}^{-1}\right]\right)>23.6$ (8 objects);

2. ten of thirteen RQQs with a detected ENLR (77\%) have a radio detection, while only five of fourteen RQQs with a nondetected ENLR (40\%) are detected in the radio;

3 . in terms of radio-loudness, we detected an ENLR only around $26 \%$ of the RQQs with $R<1$, but around $87.5 \%$ of the QSOs with $1<R<10$.

The ENLR luminosities of RLQs (not included in Fig. 16) are of the order of their radio-quiet counterparts, although the integrated radio luminosity is more than two orders of magnitude higher. Whether this is an intrinsic property or caused by the limited FoV of our IFU observation is something we are not able to say at present.

Sulentic et al. (2000) found that the broad $\mathrm{H} \beta$ line width of RLQs is almost always $>4000 \mathrm{~km} \mathrm{~s}^{-1}$, and defined two different populations of QSOs. Population A with $F W H M_{\mathrm{H} \beta} \leq$ $4000 \mathrm{~km} \mathrm{~s}^{-1}$ is entirely dominated by RQQs and Population B with $F W H M_{\mathrm{H} \beta}>4000 \mathrm{~km} \mathrm{~s}^{-1}$ contains all RLQs and a number of RQQs with otherwise similar characteristics. These two populations represent opposite regimes along the famous Eigenvector 1 (Boroson \& Green 1992), which ultimately links the width of $\mathrm{H} \beta$, the strength of the Fe II complexes and the

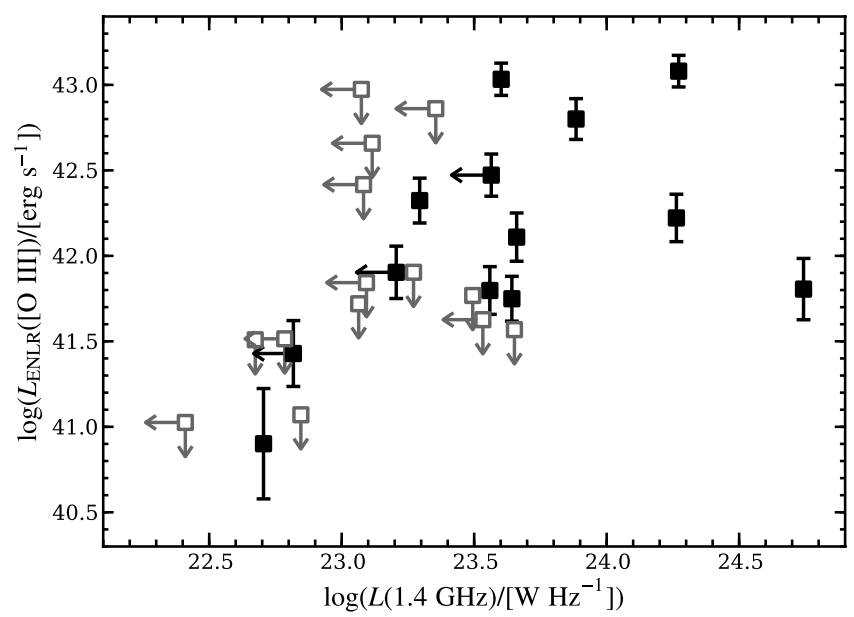

Fig. 16. [O III] luminosity of the ENLR against radio luminosity at $1.4 \mathrm{GHz}$. Black solid symbols refer to objects with detected and grey open symbols to objects with undetected ENLR (upper limits).

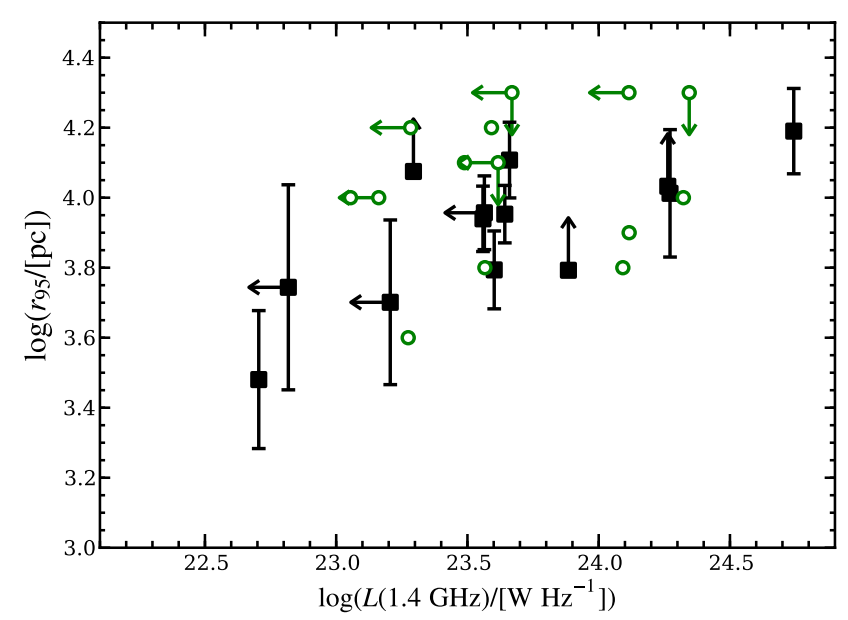

Fig. 17. ENLR size as a function of radio luminosity at $1.4 \mathrm{GHz}$. Measurements for our RQQs with detected ENLR are indicated by the black filled symbols and open green symbols denote measurements for type 2 QSOs as reported by Greene et al. (2011).

relative strength of $[\mathrm{O} \mathrm{III}]$ with respect to $\mathrm{H} \beta$. The physical drivers for this sequence in parameter space are thought to be $M_{\mathrm{BH}}$ and $\lambda$ (Boroson 2002; Marziani et al. 2003). In $\mathrm{Hu} 08$ we emphasised that a luminous ENLR is preferentially found around QSOs with $F W H M_{\mathrm{H} \beta}>4000 \mathrm{~km} \mathrm{~s}^{-1}$ and weak Fe II emission that would correspond exclusively to Population A. Our extended sample now contains a few objects, such as the radio-intermediate QSOs PG $1612+261$ and SDSS $1655+2146$, which are surrounded by a luminous ENLR but have a $F W H M_{\mathrm{H} \beta}<3000 \mathrm{~km} \mathrm{~s}^{-1}$ and therefore do not correspond to Population A as one would have naïvely expected. This is in agreement with the results of Zamfir et al. (2008) who found that radio-intermediate QSOs also cover the full range in broad $\mathrm{H} \beta$ widths like RQQs and are not restricted to Population A like RLQs.

Furthermore, Leipski et al. (2006) reported a significant trend between the size of the radio jet in RQQs and the ENLR. Because the radio emission is resolved only for very few of our QSOs, we investigate instead whether the ENLR size depends on the radio luminosity (Fig. 17) including the recent measurements for type 2 QSOs by Greene et al. (2011). Only a weak trend between the two quantities seems to be present if there is 
B. Husemann et al.: The properties of the extended warm ionised gas around low-redshift QSOs

one at all. Given our tentative evidence that the radio luminosity is more strongly correlated with the ENLR luminosity than its size, we speculate that a radio jet increases the covering factor of the gas for the ionising photons of the nucleus, reaching out to larger distances. This would well match with the scenario proposed by Hopkins et al. (2010), who argued that a weak outflow in a hot diffuse medium can possibly increase the impact of the AGN on the host galaxy via a two-stage process. First, cold molecular gas clouds expand or are destroyed by the outflow induced pressure gradients and instabilities, which could be generated by a radio jet. Second, the molecular clouds are more efficiently illuminated by the QSO radiation, because of their increased cross-sections and covering factor, so that the AGN can dissipate more energy into the medium.

\section{Kinematics of the extended ionised gas}

\subsection{Construction of $2 D$ velocity fields}

In order to study the kinematics of the EELR we used the brightest emission lines to infer the $2 \mathrm{D}$ radial velocity distribution of the ionised gas. We modelled the [O III] $\lambda \lambda 4960,5007$ doublet and/or the $\mathrm{H} \alpha$ emission line adopting a simple Gaussian line profile in each spaxel of the nucleus-subtracted datacubes. The line widths of the doublet lines were coupled and their line ratio was fixed to the theoretical value. We also modelled each spectrum assuming a Gauss-Hermite line profile up to the fourth order following van der Marel \& Franx (1993) to identify asymmetric line profiles in the EELR not caused by noise. Asymmetric lines are not frequently found in the EELR of our QSO sample (see co-added spectra in Fig. 7). In the following, we first present the $2 \mathrm{D}$ velocity fields for the bulk gas motion only. When line asymmetries are significant we replace the radial velocity of the single Gaussian fit with the mean radial velocity of the Gauss-Hermite result at that location. In addition to the $2 \mathrm{D}$ velocity maps, we constructed synthetic "longslit" curves along a certain PA directly from the maps in a geometrically manner. We averaged the velocities of all spaxel that are covered by a hypothetical slit of 1.'3 width at an angular separation of $0 . ' 5$ along the slit axis. The QSOs PG 1700+518, PHL 1811 and PG 2130-099 were excluded from this 2D analysis, as their EELR are too small in spatial extent. Still, it is worthwhile to note that we measured a redshifted radial velocity of $\sim 800 \mathrm{~km} \mathrm{~s}^{-1}$ for the EELR structure of PG $1700+518$ (cf. Table 7). Furthermore, we needed to exclude RBS 219 and SDSS J0155-0857, for which the low S/N of the extended emission lines did not allow a spatially resolved analysis.

We used the narrow [O III] line in the QSO spectrum to estimate the systemic redshifts of the QSO host galaxies. Previous studies showed that the spatially unresolved [O III] line is often systematically blueshifted in QSOs (e.g. Eracleous \& Halpern 2004; Boroson 2005; Komossa \& Xu 2007; Villar-Martín et al. 2011a), which has been interpreted as a signature for circumnuclear outflows. Whether such outflows commonly extend out to $\mathrm{kpc}$ scales is one of the issues we want to address. We present the $2 \mathrm{D}$ velocity fields in three categories, (i) apparently large scale rotational motions (Fig. 18), (ii) velocity fields of morphologically identified major mergers (Fig. 19), and (iii) miscellaneous velocity fields (Fig. 20), which we discuss separately in the following.

\subsubsection{EELRs dominated by rotational motion}

We find signatures of large scale rotational motion around I Zw 1, PG 0052+251, PKS 1545+21, PG 1612+261,
Table 10. Kinematic properties of rotational velocity fields.

\begin{tabular}{lccccc}
\hline \hline Name & $\begin{array}{c}\mathrm{PA}^{a} \\
{\left[{ }^{\circ}\right]}\end{array}$ & $\begin{array}{c}\Delta v_{r}{ }^{b} \\
{\left[\mathrm{~km} \mathrm{~s}^{-1}\right]}\end{array}$ & $\begin{array}{c}v_{0}{ }^{c} \\
{\left[\mathrm{~km} \mathrm{~s}^{-1}\right]}\end{array}$ & $\begin{array}{c}R^{d} \\
{\left[{ }^{\prime \prime} / \mathrm{kpc}\right]}\end{array}$ & $\begin{array}{c}v_{r}(R)^{e} \\
{\left[\mathrm{~km} \mathrm{~s}^{-1}\right]}\end{array}$ \\
\hline I Zw 1 & -28 & 88 & 704 & $3 / 3.5$ & 166 \\
PG 0052+251 & 2 & 94 & 134 & $3 / 8.1$ & 284 \\
PKS 1545+210 & -46 & 165 & 3 & $2 / 8.2$ & 299 \\
PG 1612+261 & 6 & 57 & 39 & $3 / 7.0$ & 119 \\
HE 2158-0107 & -49 & 17 & 5 & $2 / 6.9$ & 49 \\
HE 2353-0420 & 20 & 70 & -25 & $2 / 7.3$ & 138 \\
\hline
\end{tabular}

Notes. ${ }^{(a)}$ Position angle along the highest velocity gradient. ${ }^{(b)}$ Radial velocity gradient along the major kinematic axis per arcseconds. (c) Radial velocity zero point that the QSO position with respect to the rest-frame defined by the nuclear [O III] line. ${ }^{(d)}$ Characteristic radius from the centre used for a dynamical mass estimate. ${ }^{(e)}$ Radial velocity at the radius given given in (d).

HE 2158-0107 and HE 2353-0420. The corresponding gas velocity fields are shown in Fig. 18. Because the turn-over point in the velocity curve is not reached in most cases, we modelled the synthetic longslit curves with a straight line $\left(v_{r}=\Delta v_{r} \cdot r+v_{0}\right)$ to search for the major kinematic axis with the steepest velocity gradient $\Delta v_{r}$ among all $-90^{\circ}<\mathrm{PA}<90^{\circ}$ in steps of $3^{\circ}$. The velocity curves along this kinematic major axis are also shown in Fig. 18 and the corresponding parameters are given in Table 10. The majority of these systems (4/6) are morphological disc-dominated galaxies for which the kinematic major axis is well aligned with the photometric major axis within $\pm 14^{\circ}$ (rms). Thus, we suggest that the ionised gas is following the gravitational motion of the stellar disc in these cases.

To verify the reliability of our velocity fields, considering that the bright QSO has been subtracted before, we compare our ionised gas kinematics of I Zw 1 with ${ }^{12} \mathrm{CO}(1-0)$ data on scales of $1^{\prime \prime}-14^{\prime \prime}$ (Schinnerer et al. 1998). A radial H $\alpha$ velocity of $v_{r}=$ $166 \pm 22 \mathrm{~km} \mathrm{~s}^{-1}$ at a distance of $2^{\prime \prime}$ is in good agreement with a velocity of $180 \mathrm{~km} \mathrm{~s}^{-1}$ at $1.6^{\prime \prime}$ reported by Schinnerer et al. (1998) from the CO kinematics. Such a comparison between CO and ionised gas kinematics is only possible for I $\mathrm{Zw} 1$, because $\mathrm{CO}$ observations with the required spatial resolution and depth do not yet exist for other QSOs in our sample.

PKS $1545+210$ and possibly also HE 2158-0107 are bulgedominated host galaxies for which the inclinations of the putative rotating gas discs are unknown. It is unlikely that the gas kinematics are caused by AGN-driven bipolar outflows, because the radio jet of PKS $1545+210$ is oriented perpendicular to the kinematic major axis (see Sect. 7.2), and HE 2158-0107 is a RQQ with a low radio luminosity. Interestingly, an apparent companion galaxy is located $\sim 3^{\prime \prime}$ to the West of PKS $1545+210$ in the direction of the kinematic major axis and HE 2158-0107 features an extended tail of gas. Both case are suggestive for the accretion of gas from companions or the environment itself (see the case of HE 2158-0107 in Husemann et al. 2011), which will govern the rotation axis of the gas.

A direct comparison of the $\mathrm{H} \alpha$ and [O III] kinematics is only possible for $\mathrm{PG} 0052+251$. We find that the [O III] kinematics does not match the clear rotational $\mathrm{H} \alpha$ velocity field with an offset of up to $\sim 100 \mathrm{~km} \mathrm{~s}^{-1}$. This kinematic separation supports our interpretation of the emission-line ratios in Sect. 5.3, in which the $\mathrm{H} \alpha$ emission originates dominantly from $\mathrm{H}$ II regions in the discs, whereas the [O III] emission more often represents AGN ionisation cones. 

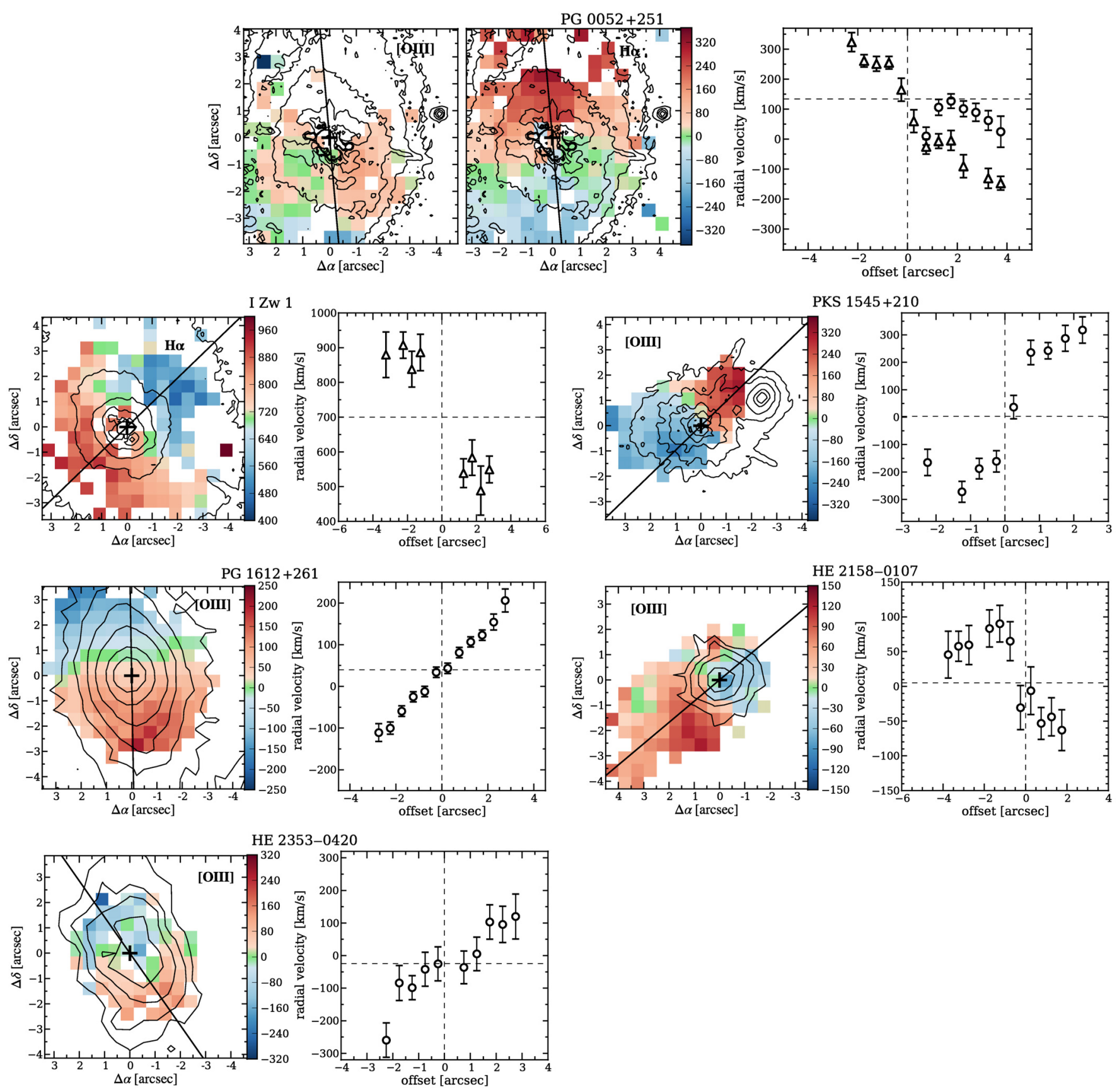

Fig. 18. 2D velocity maps for objects with apparently large-scale rotational motion gas motion. The continuum light distribution of broad-band images are overplotted as thin solid black contours for comparison. A "longslit" velocity curves along the apparent major kinematic axis is extracted from the maps as described in the text. A thick solid black line indicates the orientation of the major kinematic axis. The radial velocity are estimated for each spaxel by modelling the corresponding emission line with a Gaussian profile. The vertical dashed line highlights the position of the QSO along the synthetic slit and the horizontal dashed line indicates the measured velocity zero-point of the velocity curve.

\subsubsection{Velocity fields of interacting QSO host galaxies}

In Fig. 19 we present the gas velocity fields of Mrk 1014, SDSS $\mathrm{J} 1131+2632$ and PKS 2349-012, which we identified to be ongoing major mergers based on their optical continuum images. Because of the complex mass distribution and tidal forces, we do not necessarily expect a clear rotational velocity pattern in these systems.

Our PMAS observation of Mrk 1014 covers only the inner $10 \mathrm{kpc}$ part of the QSO host (cf. Fig. 7), so that we cannot trace the bulk motion of the ionised gas across the whole galaxy. The [O III] velocity curve along the EELR major axis appears to be peculiar. The symmetric curve has two sharp peaks in the radial velocity at \pm 2 " distance from the nucleus at which the radial motion changes from receding to approaching and vice versa. These features are associated with a region of strong jet-cloud interactions as discussed below in Sect. 7.2, likely leading to non-relaxed bulk motion of the gas.

The EELR of SDSS J1131+2632 is very elongated, and we can only study the kinematics in a part of the galaxy similar to Mrk 1014. The most striking feature is a giant shell or arc of ionised gas, 4" South-East of the nucleus, offset by $352 \pm 33 \mathrm{~km} \mathrm{~s}^{-1}$ from the systemic velocity in the rest-frame. This is a unique feature among all the QSO in our sample and 

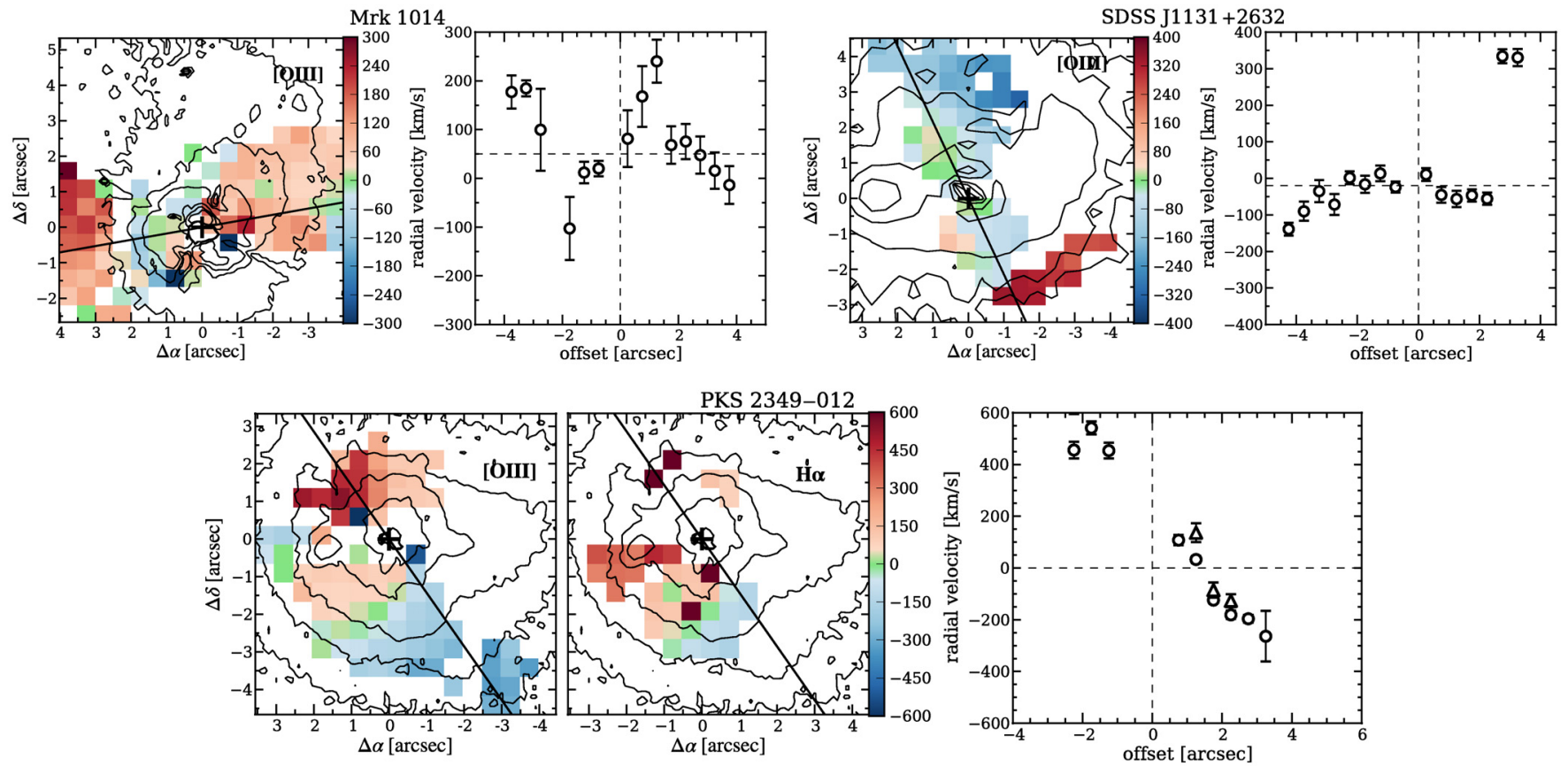

Fig. 19. Same as Fig. 18 showing the velocity fields of the 3 QSO hosts morphologically classified as ongoing major mergers.

does not correspond to any continuum substructure in the broadband image of the complex merging system. One possibility is that the gas belongs to a faint companion galaxy illuminated by the AGN radiation.

A strong velocity gradient is detected in the ionised gas of PKS 2349-014. The main axis of this gradient follows the large curved wisps that were identified in the HST images by Bahcall et al. (1995). Thus, the gas motion is clearly driven by gravitation. However, the radio-jet axis of this RLQ is oriented North-South, and the radio jet seems to kinematically disturb the ionised gas at the interception point of jet and ENLR.

\subsubsection{Miscellaneous velocity fields}

The radial velocity fields of the remaining four QSO hosts, PG 0026+129, SDSS J0836+4426, PKS 1217+023 and SDSS J1655+2146, are either asymmetric or lack a significant velocity gradient across the galaxy. All four host galaxies appear to be bulge-dominated, in which the gas could be kinematically hot and slowly rotating. However, the SDSS images of $\mathrm{J} 1655+2146$ and $\mathrm{J} 0836+4426$ do not rule out a disc component, which would need to be studied with higher spatial resolution imaging. We do not find any strong evidence for extreme kinematic features that would indicate non-gravitational motion.

\subsection{Evidence for jet-cloud interactions on $\mathrm{kpc}$ scales}

We already discussed a close connection between the ENLR and the presence and morphology of radio jets emerging from the nucleus in Sect. 6. To follow up on this we now address the question whether the kinematics of the ionised gas is also significantly influenced by the jets.

We detect significantly asymmetric [O III] line profiles close to the nucleus of Mrk 1014 and PG 1612+261 right at the location of their known radio hot spots (Fig. 21). The line width and radial velocity offset are $508 \pm 84 \mathrm{~km} \mathrm{~s}^{-1}$ and $-1012 \pm 48 \mathrm{~km} \mathrm{~s}^{-1}$ for Mrk 1014 and $424 \pm 24 \mathrm{~km} \mathrm{~s}^{-1}$ (FWHM) and $-220 \pm 20 \mathrm{~km} \mathrm{~s}^{-1}$ for PG $1612+261$ at region $\mathrm{A}$, respectively, indicating clearly non-gravitational motions. In addition to the blue-shifted component in Mrk 1014, a broad redshifted component is blended with the [O III] narrow component. The extended ionised gas kinematics of Mrk 1014 was already studied in detail by means of long-slit (Leipski \& Bennert 2006) and IFU spectroscopy (Fu \& Stockton 2009). Our data for this object are fully supporting their results. In addition, SDSS J0836+4426 and SDSS J1655+2146 similarly show asymmetric [O III] line profiles close to the nucleus, which we separated into two different components. It is possible that jet-cloud interactions play a significant role in producing these complex kinematic feature as well given their significant integrated radio luminosity of $2 \times 10^{24} \mathrm{~W} \mathrm{~Hz}^{-1}$ and $4 \times 10^{23} \mathrm{~W} \mathrm{~Hz}^{-1}$, respectively. Yet, no interferometric radio images are available for these two objects that would be needed to confirm a clear association between radio emission and line asymmetries for a jet-cloud interaction scenario.

The EELR of PG $1700+518$ is almost coincident with the south-east radio hot spot, considering the low spatial resolution of the optical compared to the radio observation. It is tempting to speculate that the radio-jet is accelerating the gas to the observed velocities $\left(\sim 800 \mathrm{kms}^{-1}\right)$. On the other hand, the EELR may also belong to a companion galaxy or a starburst region as part of the merger, because a knot in the $H$-band adaptive optics image was identified by Márquez \& Petitjean (2003). The brightest region of the EELR around the RQQ PG 2130+099 is matching with the southern hot spot of the triple radio source. However, we could not find any kinematic impact on the gas as in the other cases.

The [O III] kinematics of the major merger host of the RLQ PKS 2349-012 are mainly quiescent. However, we find redshifted [O III] emission by $400 \pm 70 \mathrm{~km} \mathrm{~s}^{-1}$ with an intrinsic line width of $\sim 700 \mathrm{~km} \mathrm{~s}^{-1}$ (FWHM) at the interception point with the radio jet axis ( $2^{\prime \prime}$ North of the nucleus). This highlights the impact of the radio jet on the kinematics at the interception point, but not in the surrounding regions.

The RLQs PKS 1217+023 and PKS $1545+210$ reside both in bulge-dominated galaxies. The jet axis of PKS $1217+023$ 

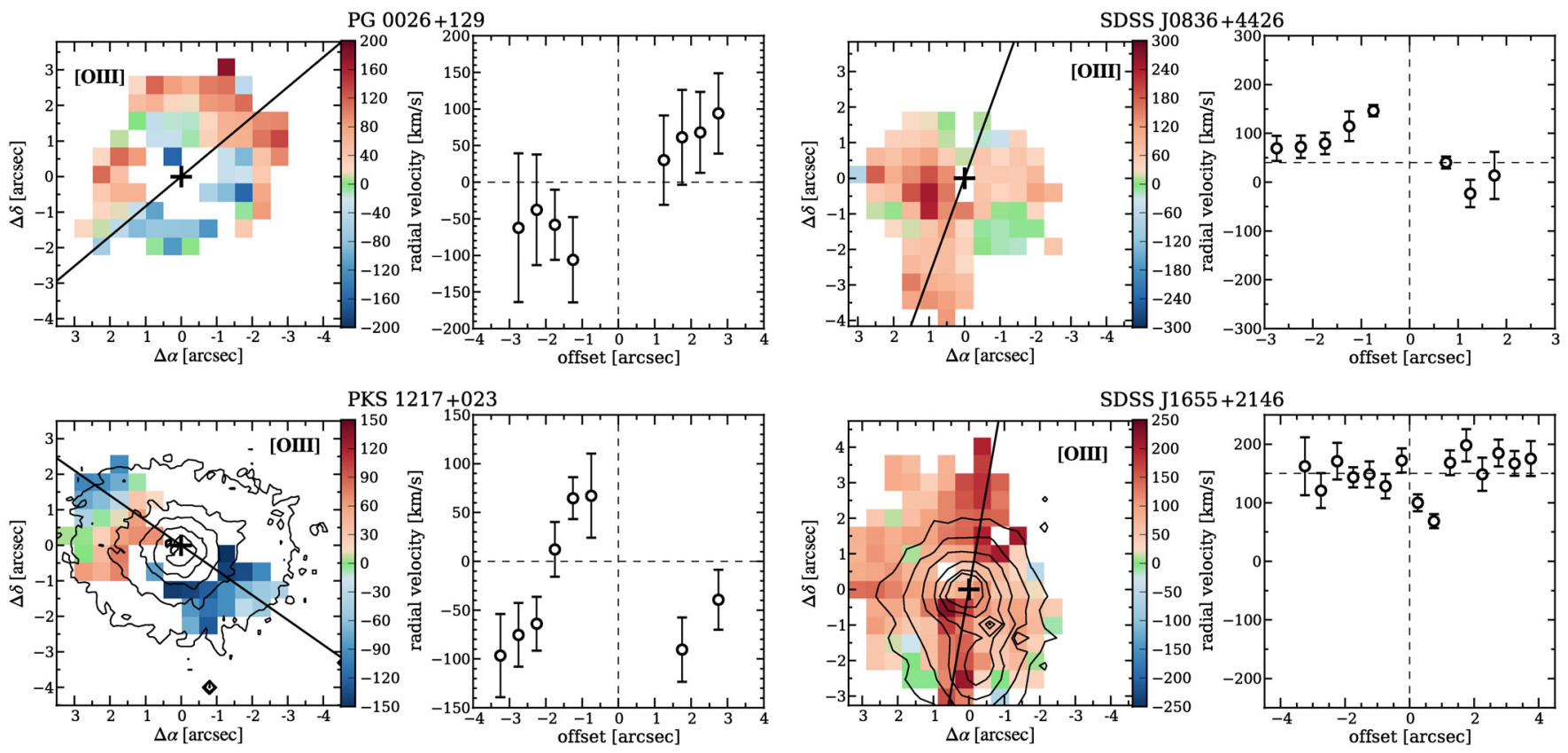

Fig. 20. Same as Fig. 18, presenting the miscellaneous velocity fields of the remaining objects.

is aligned with the EELR major axis along which the velocity curve does clearly not agree with rotational pattern. Whether the gas is directly interacting with the jet remains uncertain because of the narrow line width and radial velocity $<200 \mathrm{~km} \mathrm{~s}^{-1}$. An interaction of the jet with the EELR around PKS $1545+210$ is most unlikely because the radio jet is aligned with the rotation axis of the gas disc. Such rotating gas discs are common in low-redshift radio galaxies (e.g. Kotanyi \& Ekers 1979; Baum et al. 1992) with Centaurus A being the most prominent example.

To summarize these individual results, in Fig. 22 we compare the maximum radial [O III] EELR velocity offset with respect to the zero-point of the velocity curves against the radio luminosity for all RQQs in our sample and that of Greene et al. (2011). A tentative trend is emerging that the radial velocity of the highly ionised gas on kpc scales increases with the radio luminosity, i.e. jet power, even in RQQs. Despite some contamination with purely rotational motions at low radio luminosity and the unknown geometry and inclination of the gas motion, this supports the notion of a the systematic influence of radio jets on the ionised gas kinematics on host galaxy scales. A quite similar trend was already reported by Müller-Sánchez et al. (2011) for sub-kpc scales in lower luminosity Seyfert galaxies based on near-infrared IFS. Nevertheless, our inferred radial velocities are in the majority of cases below the escape velocity, which is of the order of 500-1000 $\mathrm{km} \mathrm{s}^{-1}$ (see Greene et al. 2011). Such a trend with increasing radio luminosity may be expected as the jets will carry mechanical energy and momentum of which a certain fraction will certainly be dissipated in the surrounding gas. Radio-loud AGN are therefore known to exhibit jetcloud interactions that accelerate the gas to significant velocities (e.g. Baldwin et al. 1980; Holt et al. 2003; Sánchez et al. 2004a; Morganti et al. 2005; Christensen et al. 2006; Nesvadba et al. 2006). On the other hand, detailed studies of some RQQs (e.g. SDSS J1356+1026, Greene et al. 2012) and ULIRGs (e.g. Mrk 231, Rupke \& Veilleux 2011) have shown that a radio jet alone is energetically insufficient to power high-velocity outflows present in these objects. The lack of high velocity ionised gas clouds in the majority of luminous RQQs is not necessarily in contrast with those observations. It could be that radio jets dominate the cloud acceleration even energetically in the majority of cases, but with some particular exceptions. An alternative explanation could be that radio jets significantly increase the effective energy dissipation of the QSO radiation into the ambient medium. We mentioned already in Sect. 6.5 the possibility that a jet might decrease the clumpiness of the surrounding medium so the QSO radiation can act more strongly as proposed by Hopkins et al. (2010) in their two-stage AGN feedback model.

\subsection{The incidence of outflows among RQQs: no evidence for AGN feedback?}

AGN feedback has generally become an important ingredient in cosmological simulations of galaxy formation to efficiently stall star formation on short time scales. In these models either a significant amount of gas is expelled from the host galaxy by AGN outflows (e.g. Sanders et al. 1988; Hopkins et al. 2005) or the gas in the galaxy halo is episodically heated by radio jets to prevent further condensation of cold gas (e.g. Croton et al. 2006; Bower et al. 2006; Best et al. 2006). Clear observational evidence for the latter process are the intra-cluster X-ray cavities driven by radio jets of the brightest cluster galaxies (see Cattaneo et al. 2009; Fabian 2012, and references therein). On the other hand, observational signatures for AGN driven outflows are still scarce, and their interpretations are a subject of debate.

Galactic superwinds with outflow velocities of up to $1000 \mathrm{~km} \mathrm{~s}^{-1}$ in the ionised gas have been detected in Ultra Luminous Infrared Galaxies (ULIRGs) (e.g. Heckman et al. 2000; Rupke et al. 2005; Rupke \& Veilleux 2011; Harrison et al. 2012; Westmoquette et al. 2012) and massive post-starburst galaxies (Tremonti et al. 2007). These extraordinary outflows may be driven by the radiation of the central AGN with a comparably low contribution from the starburst. Whether AGNdriven outflows are solely responsible for such outflow velocities is however questioned by recent observation of poststarburst galaxies that display high-velocity outflows without clear evidence for AGN (Diamond-Stanic et al. 2012). This is consistent with a comparison study of the radial gas velocity in X-ray selected AGN hosts and post-starburst galaxies 

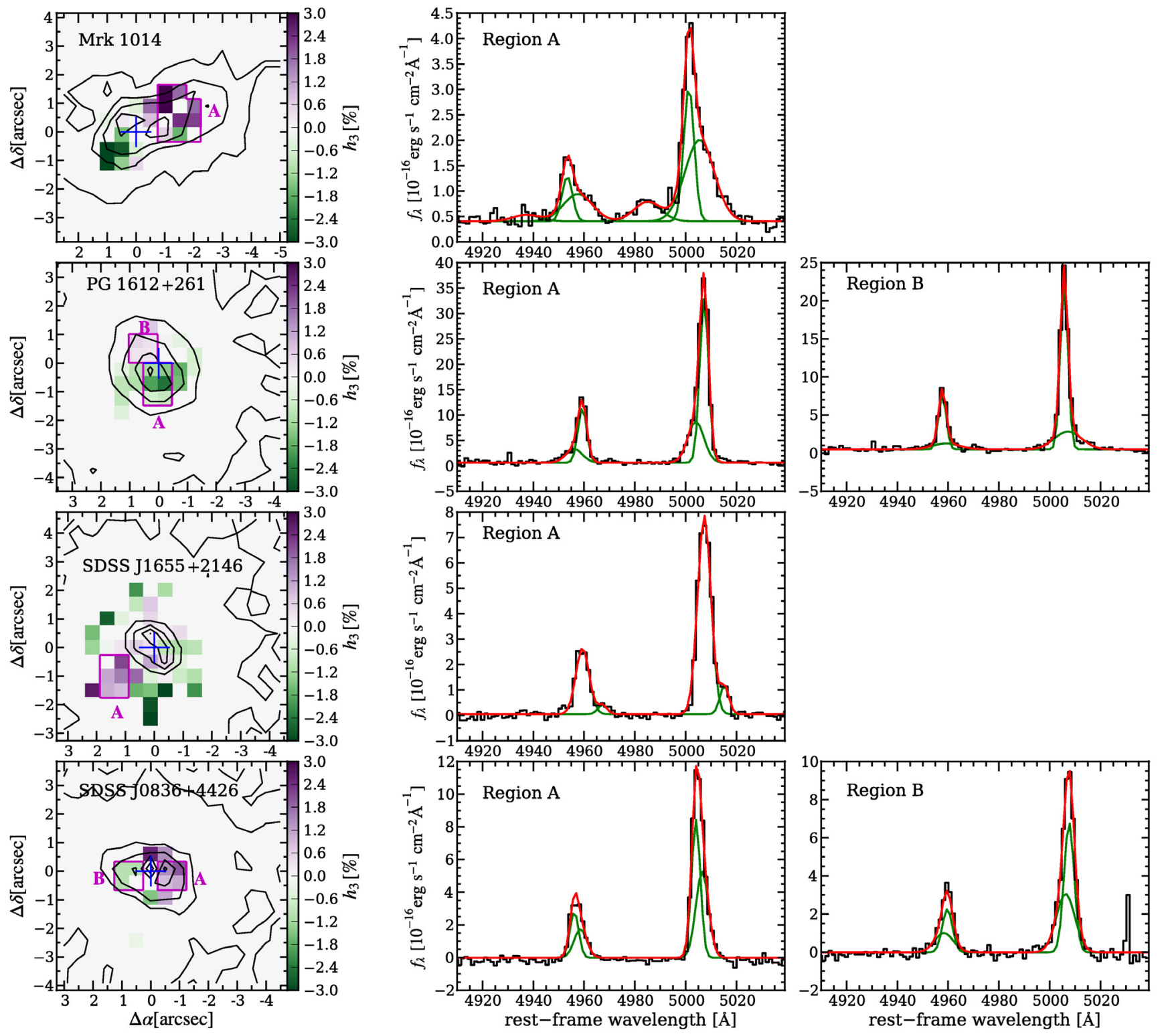

Fig. 21. Left panel: strength of the third Gauss-Hermite coefficient $h_{3}$ representing an asymmetry in the line profile found to be significant only in 4 galaxies, i.e. Mrk 1014, PG 1612+261, SDSS J1655+2146, and SDSS J0836+4426. The black contours represent the [O III] surface brightness distribution for comparison and the blue cross marks the QSO position for reference. Right panel: modelling of [O III] $\lambda 4960,5007$ doublet line in the co-added spectra (of the spaxels in the magenta box on the left) with two Gaussian components. The red line is the model to the measured spectrum shown in black, and the green line represents the individual Gaussian components.

by Coil et al. (2011). They concluded that while typical outflow velocities of $200 \mathrm{~km} \mathrm{~s}^{-1}$ are seen in both samples, the winds are likely driven by vigorous star formation and not by AGN. Furthermore, ionised gas with radial velocities exceeding more than $500 \mathrm{~km} \mathrm{~s}^{-1}$ have been detected mainly around radio-loud AGN (e.g. Nesvadba et al. 2006; Holt et al. 2008; Fu \& Stockton 2009), which can be attributed to the mechanical energy of jet.

In our sample of 31 low-redshift QSOs we find signatures of outflowing $\left(\gtrsim 400 \mathrm{~km} \mathrm{~s}^{-1}\right)$ ionised gas on kpc scales only in $3 \mathrm{ob}-$ jects. In all these cases we identify a radio jet that is most likely driving the outflow. Quiescent gas kinematics, or no ionised gas at all, are characteristic for the rest of the sample. Similarly, Greene et al. (2011) found extended quiescent kinematics in the ionised gas of all but one type $2 \mathrm{QSO}$, but they attempt to use the wings of the [O III] line to argue for the presence of some level of AGN feedback. The low outflow detection rates agree with the result of Krug et al. (2010), who compared the radial velocity of the $\mathrm{NaID}$ absorption line in the spectra of infrared-faint $\left(S F R<10 M_{\odot} \mathrm{yr}^{-1}\right)$ Seyfert galaxies at $z<0.05$ and reported an outflow detection rate of $6 \%$ for Seyfert 1 and $18 \%$ for Seyfert 2 galaxies, compared to more than $50 \%$ for starburst galaxies.

Our observations clearly show a lack of ionised gas outflows in radio-quiet AGN at low redshift, which may challenge the incorporation of AGN feedback in some theoretical models. However, no ionised gas at all is found around almost half of the QSOs in our sample. These QSOs typically show weak [O III] emission in their nuclear spectra, which could be interpreted in two ways: (i) the majority of gas has already been expelled from the host galaxy (e.g. Netzer et al. 2004), (ii) the majority of gas within the host galaxy is not sufficiently ionised by the AGN, or (iii) the galaxies are intrinsically poor of gas. With the advent of new radio/sub-mm telescopes, like the Atacama 


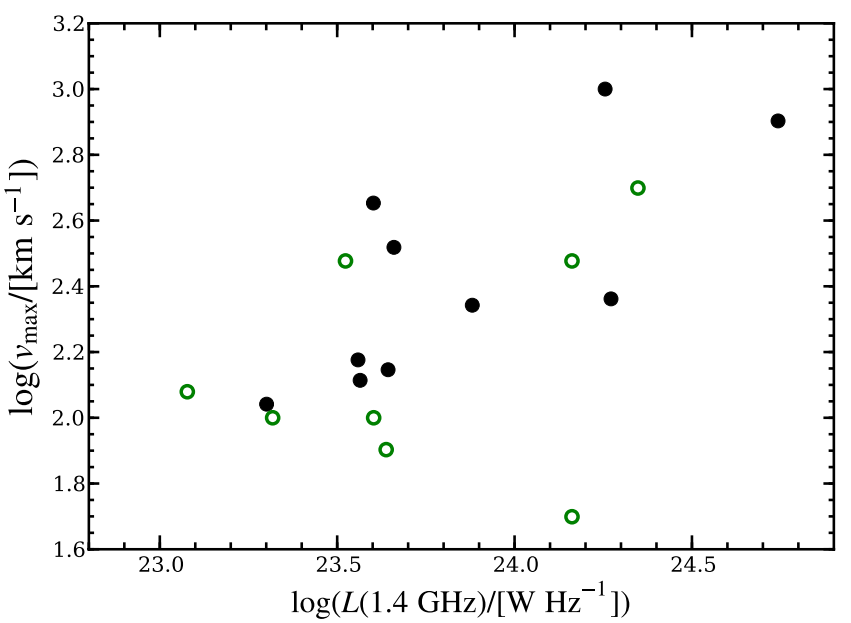

Fig. 22. Extended maximum [OIII] radial velocity in the ENLR of RQQs as a function of radio luminosity at $1.4 \mathrm{GHz}$. Only objects with detected radio emission are included in the diagram. Data from our IFU study of RQQs are indicated by the black filled symbols and open green symbols are from the long-slit data of type 2 QSOs (Greene et al. 2011).

Large Millimetre Array (ALMA), we have the opportunity to study the amount, distribution, and kinematics of the cold molecular gas at a sufficient spatial and spectral resolution. Given that the molecular gas is the seed of star formation, it may be the more direct tracer for AGN feedback processes than the ionised gas phase.

\section{Conclusions}

In this paper we presented a detailed analysis of the extended ionised gas around a large sample of 31 low-redshift QSOs observed with the PMAS integral field spectrograph on the $3.5 \mathrm{~m}$ telescope at the Calar Alto observatory. This is currently the largest sample of low-redshift type 1 QSOs observed with integral field spectroscopy in the optical wavelength regime. In Husemann et al. (2008) we already studied the presence of extended ionised gas in relation to the spectral properties of the QSO nucleus based on a subsample already observed at that time. With the full sample now in hand, we investigated the ionisation states, luminosities, sizes of the extended narrow line regions (ENLRs), and the kinematics of the ionised gas. In order to separate the unresolved QSO emission from extended host galaxy emission in the IFU data we introduced our new software tool QDeblend ${ }^{3 D}$. The main results of our analysis can be summarised as follows:

- We detect extended ionised gas around 19 of 31 QSOs $(61 \%)$, of which 13 are ionised mainly by the QSO (the ENLR), 3 are $\mathrm{H}$ II-like regions produced by ongoing star formation, and the remaining 3 objects show signatures of both types in different extended regions.

- Previous ENLR sizes obtained from HST narrow-band images are underestimated by at least a factor of 2 because of the much shallower surface brightness limits. Nine of sixteen ENLRs in our sample are elongated which means that longslit observations will strongly depend on the slit orientation. In the worst case they will underestimate the size by up to a factor of 6 for individual object, and a factor of 2 in the mean.

- The typical size of the ENLR is $r_{95}=10 \pm 3 \mathrm{kpc}$ at a median [O III] luminosity $\log \left(L([\mathrm{O}\right.$ III $\left.]) /\left[\mathrm{ergs}^{-1}\right]\right)=42.7 \pm 0.15$ of our QSO sample. Recent studies of type 2 QSOs at comparable luminosity by Greene et al. (2011) and Fu et al. (2012) obtained very similar ENLR sizes compared to our type 1 QSOs. This implies that the ENLR size-luminosity relation for type 1 and type 2 AGN cannot be as different as implied by the results of Schmitt et al. (2003b). The reported discrepancy between their extrapolated ENLR sizes of luminous type 2 and type 1 QSOs is therefore obsolete.

- We find that the QSO continuum luminosity correlates much better with the ENLR size than does the total [O III] luminosity, while the [O III] luminosity of the ENLR is totally uncorrelated with the QSO continuum luminosity. Thus, we argue that the [O III] luminosity is not a good proxy for the intrinsic luminosity of the QSO and that the amount of ionising photons does not determine the luminosity of an ENLR alone. Either the amount and distribution of gas is quite different in the host galaxies or other factors such as the impact of radio jets play a additional role. This is supported by a trend that the ENLR luminosity increases with radio luminosity. We therefore caution to use type 2 AGN to study the size-luminosity relation given that the [O III] luminosity is a secondary and less accurate proxy for the intrinsic luminosity of the nucleus (e.g. Punsly \& Zhang 2011). Additionally, type 2 QSOs are often found to be radio-intermediate objects and have a much higher incidence of flat-spectrum radio source (Lal \& Ho 2010), which might cause a certain selection effect for type 2 QSOs if the radio jets enhance the [O III] luminosity relative to the bolometric AGN luminosity.

- Signatures of presumably rotational gas kinematics are identified in 6 host galaxies of which 4 are disc-dominated. Signatures of non-gravitational kinematics have been found in in 5 cases for specific regions within the EELRs among which 3 show radial velocity greater than $\gtrsim 400 \mathrm{~km} \mathrm{~s}^{-1}$. Given the coincidences with extended radio sources, we argue that jet-cloud interactions are the most likely origin for the disturbances in the kinematics in the majority of cases. However, the detection rate and radial velocities of the outflows are much lower than reported for starburst galaxies or radio-loud AGN.

One of the main results of our study is the low detection rate of gas outflows around low-redshift radio-quiet QSOs (RQQs), which is in agreement with similar results from type 2 QSOs and infrared faint Seyfert galaxies. Taken all together this is evidence that massive AGN-driven bulk outflows of gas are not ubiquitous around RQQs at low redshifts. Signatures of AGN feedback are mainly observed in radio-loud AGN which can expel the gas from the host galaxy or even drive large cavities in the intra-cluster medium. The lack of gas outflow signatures in RQQs may challenge some the theoretical AGN feedback scenarios in which the expulsion of gas is essential to stall star formation on short time scales. One explanation could be that the QSOs investigated here, while among the most luminous at these low redshifts, are still too faint compared to their more ultraluminous high-redshift counterparts, to display the same strong signature of vigorous outflows in the ionised gas (Cano-Díaz et al. 2012; Maiolino et al. 2012). But our RQQs may also be in late stages of their evolution, after most of the gas has either been heated or was already expelled from the host galaxies considering the presumed time delay between the peak of starburst and AGN activity of around a few $100 \mathrm{Myr}$ (Li et al. 2008; Wild et al. 2010). On the other hand, the ENLR sizes, luminosities and kinematics provide evidence for the general importance of radio jets to increase the covering factor of the ambient gas for the ionising photons of the nucleus in a pre-stage of feedback. To understand AGN feedback processes in RQQs therefore still remains an open issue. 
B. Husemann et al.: The properties of the extended warm ionised gas around low-redshift QSOs

Acknowledgements. We thank the referee Jenny Greene for a prompt report with many valuable comments that clearly improved the quality of the article. Additionally, we thank Vardha Nicola Bennert for kindly providing us their analysed HST narrow-band images, Christian Leipski for sending us their reduced radio maps, and Hai Fu for making their unpublished ENLR size measurements available to us. B.H. and L.W. acknowledge financial support by the DFG Priority Program 1177 "Witnesses of Cosmic History: Formation and evolution of black holes, galaxies and their environment", grant Wi 1369/22. K.J. is funded through the DFG Emmy NoetherProgram, grant JA 1114/3-1. Part of the data used in the paper originate from observation made with the NASA/ESA Hubble Space Telescope, and obtained from the Hubble Legacy Archive, which is a collaboration between the Space Telescope Science Institute (STScI/NASA), the Space Telescope European Coordinating Facility (ST-ECF/ESA) and the Canadian Astronomy Data Centre (CADC/NRC/CSA). Funding for the SDSS and SDSS-II was provided by the Alfred P. Sloan Foundation, the Participating Institutions, the National Science Foundation, the US Department of Energy, the National Aeronautics and Space Administration, the Japanese Monbukagakusho, the Max Planck Society, and the Higher Education Funding Council for England. The SDSS was managed by the Astrophysical Research Consortium for the Participating Institutions. This research has made use of the NASA/IPAC Extragalactic Database (NED) which is operated by the Jet Propulsion Laboratory, California Institute of Technology, under contract with the National Aeronautics and Space Administration. For the preparation of this paper we have made used of the cosmology calculator "CosmoCalc" (Wright 2006).

\section{References}

Aalto, S., Garcia-Burillo, S., Muller, S., et al. 2012, A\&A, 537, A44

Adelman-McCarthy, J. K., Agüeros, M. A., Allam, S. S., et al. 2008, ApJS, 175, 297

Allen, M. G., Dopita, M. A., \& Tsvetanov, Z. I. 1998, ApJ, 493, 571

Alonso-Herrero, A., Ramos Almeida, C., Mason, R., et al. 2011, ApJ, 736, 82

Antonucci, R. 1993, ARA\&A, 31, 473

Bahcall, J. N., Kirhakos, S., \& Schneider, D. P. 1995, ApJ, 447, L1

Bahcall, J. N., Kirhakos, S., \& Schneider, D. P. 1996, ApJ, 457, 557

Bahcall, J. N., Kirhakos, S., Saxe, D. H., \& Schneider, D. P. 1997, ApJ, 479, 642

Baldwin, J. A., Carswell, R. F., Wampler, E. J., et al. 1980, ApJ, 236, 388

Baldwin, J. A., Phillips, M. M., \& Terlevich, R. 1981, PASP, 93, 5

Baskin, A., \& Laor, A. 2005, MNRAS, 358, 1043

Baum, S. A., \& Heckman, T. 1989, ApJ, 336, 702

Baum, S. A., Heckman, T. M., Bridle, A., van Breugel, W. J. M., \& Miley, G. K. 1988, ApJS, 68, 643

Baum, S. A., Heckman, T. M., \& van Breugel, W. 1992, ApJ, 389, 208

Becker, R. H., White, R. L., \& Helfand, D. J. 1995, ApJ, 450, 559

Bell, E. F., McIntosh, D. H., Barden, M., et al. 2004, ApJ, 600, L11

Bennert, N. 2005, Ph.D. Thesis, University of Bochum, Germany

Bennert, N., Falcke, H., Schulz, H., Wilson, A. S., \& Wills, B. J. 2002, ApJ, 574, L105

Bennert, N., Jungwiert, B., Komossa, S., Haas, M., \& Chini, R. 2006a, A\&A, 459,55

Bennert, N., Jungwiert, B., Komossa, S., Haas, M., \& Chini, R. 2006b, A\&A, 456, 953

Bentz, M. C., Peterson, B. M., Pogge, R. W., Vestergaard, M., \& Onken, C. A. 2006c, ApJ, 644, 133

Bentz, M. C., Peterson, B. M., Netzer, H., Pogge, R. W., \& Vestergaard, M. 2009, ApJ, 697, 160

Best, P. N., Kaiser, C. R., Heckman, T. M., \& Kauffmann, G. 2006, MNRAS, 368, L67

Boroson, T. 2005, AJ, 130, 381

Boroson, T. A. 2002, ApJ, 565, 78

Boroson, T. A., \& Green, R. F. 1992, ApJS, 80, 109

Boroson, T. A., \& Oke, J. B. 1984, ApJ, 281, 535

Boroson, T. A., Oke, J. B., \& Green, R. F. 1982, ApJ, 263, 32

Boroson, T. A., Persson, S. E., \& Oke, J. B. 1985, ApJ, 293, 120

Bower, R. G., Benson, A. J., Malbon, R., et al. 2006, MNRAS, 370, 645

Bruzual, G., \& Charlot, S. 2003, MNRAS, 344, 1000

Cano-Díaz, M., Maiolino, R., Marconi, A., et al. 2012, A\&A, 537, L8

Capetti, A., Axon, D. J., Macchetto, F., Sparks, W. B., \& Boksenberg, A. 1996, ApJ, 469, 554

Cattaneo, A., Faber, S. M., Binney, J., et al. 2009, Nature, 460, 213

Christensen, L., Jahnke, K., Wisotzki, L., \& Sánchez, S. F. 2006, A\&A, 459, 717

Cid Fernandes, R., Stasińska, G., Schlickmann, M. S., et al. 2010, MNRAS, 403, 1036

Cisternas, M., Jahnke, K., Inskip, K. J., et al. 2011, ApJ, 726, 57

Coil, A. L., Weiner, B. J., Holz, D. E., et al. 2011, ApJ, 743, 46

Collin, S., Kawaguchi, T., Peterson, B. M., \& Vestergaard, M. 2006, A\&A, 456, 75
Condon, J. J., Cotton, W. D., Greisen, E. W., et al. 1998, AJ, 115, 1693

Croton, D. J., Springel, V., White, S. D. M., et al. 2006, MNRAS, 365, 11

Denney, K. D., Peterson, B. M., Dietrich, M., Vestergaard, M., \& Bentz, M. C. 2009, ApJ, 692, 246

Diamond-Stanic, A. M., Moustakas, J., Tremonti, C. A., et al. 2012, ApJ, 755, L26

Dopita, M. A., Kewley, L. J., Heisler, C. A., \& Sutherland, R. S. 2000, ApJ, 542, 224

Dunlop, J. S., McLure, R. J., Kukula, M. J., et al. 2003, MNRAS, 340, 1095

Eracleous, M., \& Halpern, J. P. 2004, ApJS, 150, 181

Fabian, A. C. 2012, ARA\&A, 50, 455

Falcke, H., Wilson, A. S., \& Simpson, C. 1998, ApJ, 502, 199

Fasano, G., \& Franceschini, A. 1987, MNRAS, 225, 155

Fraquelli, H. A., Storchi-Bergmann, T., \& Levenson, N. A. 2003, MNRAS, 341, 449

Fu, H., \& Stockton, A. 2007, ApJ, 664, L75

Fu, H., \& Stockton, A. 2008, ApJ, 677, 79

Fu, H., \& Stockton, A. 2009, ApJ, 690, 953

Fu, H., Yan, L., Myers, A. D., et al. 2012, ApJ, 745, 67

Gebhardt, K., Bender, R., Bower, G., et al. 2000, ApJ, 539, L13

Greene, J. E., \& Ho, L. C. 2005, ApJ, 630, 122

Greene, J. E., Zakamska, N. L., Ho, L. C., \& Barth, A. J. 2011, ApJ, 732, 9

Greene, J. E., Zakamska, N. L., \& Smith, P. S. 2012, ApJ, 746, 86

Gültekin, K., Richstone, D. O., Gebhardt, K., et al. 2009, ApJ, 698, 198

Guyon, O., Sanders, D. B., \& Stockton, A. 2006, ApJS, 166, 89

Hamilton, D., \& Keel, W. C. 1987, ApJ, 321, 211

Häring, N., \& Rix, H.-W. 2004, ApJ, 604, L89

Harrison, C. M., Alexander, D. M., Swinbank, A. M., et al. 2012, MNRAS, 426, 1073

Heckman, T. M. 1980, A\&A, 87, 152

Heckman, T. M., Lehnert, M. D., Strickland, D. K., \& Armus, L. 2000, ApJS, 129,493

Heckman, T. M., Kauffmann, G., Brinchmann, J., et al. 2004, ApJ, 613, 109

Henry, R. B. C., \& Worthey, G. 1999, PASP, 111, 919

Ho, L. C., \& Ulvestad, J. S. 2001, ApJS, 133, 77

Ho, L. C., Filippenko, A. V., \& Sargent, W. L. W. 1997, ApJS, 112, 315

Holt, J., Tadhunter, C. N., \& Morganti, R. 2003, MNRAS, 342, 227

Holt, J., Tadhunter, C., Morganti, R., et al. 2006, MNRAS, 370, 1633

Holt, J., Tadhunter, C. N., \& Morganti, R. 2008, MNRAS, 387, 639

Holt, J., Tadhunter, C. N., Morganti, R., \& Emonts, B. H. C. 2011, MNRAS, 410, 1527

Hopkins, A. M., Connolly, A. J., Haarsma, D. B., \& Cram, L. E. 2001, AJ, 122, 288

Hopkins, P. F., Hernquist, L., Cox, T. J., et al. 2005, ApJ, 630, 705

Hopkins, P. F., Bundy, K., Croton, D., et al. 2010, ApJ, 715, 202

Humphrey, A., Villar-Martín, M., Sánchez, S. F., et al. 2010, MNRAS, 408, L1

Husemann, B., Wisotzki, L., Sánchez, S. F., \& Jahnke, K. 2008, A\&A, 488, 145

Husemann, B., Sánchez, S. F., Wisotzki, L., et al. 2010, A\&A, 519, A115

Husemann, B., Wisotzki, L., Jahnke, K., \& Sánchez, S. F. 2011, A\&A, 535, A72

Jahnke, K., \& Wisotzki, L. 2003, MNRAS, 346, 304

Jahnke, K., Wisotzki, L., Sánchez, S. F., et al. 2004, AN, 325, 128

Jenkins, E. B., Bowen, D. V., Tripp, T. M., \& Sembach, K. R. 2005, ApJ, 623, 767

Kaspi, S., Smith, P. S., Netzer, H., et al. 2000, ApJ, 533, 631

Kaspi, S., Maoz, D., Netzer, H., et al. 2005, ApJ, 629, 61

Kauffmann, G., Heckman, T. M., Tremonti, C., et al. 2003, MNRAS, 346, 1055

Kellermann, K. I., Sramek, R., Schmidt, M., Shaffer, D. B., \& Green, R. 1989, AJ, 98, 1195

Kewley, L. J., Dopita, M. A., Sutherland, R. S., Heisler, C. A., \& Trevena, J. 2001, ApJ, 556, 121

Kewley, L. J., Groves, B., Kauffmann, G., \& Heckman, T. 2006, MNRAS, 372, 961

Khalatyan, A., Cattaneo, A., Schramm, M., et al. 2008, MNRAS, 387, 13

Kim, M., Ho, L. C., Peng, C. Y., et al. 2008, ApJ, 687, 767

Komossa, S., \& Xu, D. 2007, ApJ, 667, L33

Kotanyi, C. G., \& Ekers, R. D. 1979, A\&A, 73, L1

Krist, J. 1995, in Astronomical Data Analysis Software and Systems IV, eds. R. A. Shaw, H. E. Payne, \& J. J. E. Hayes, ASP Conf. Ser., 77, 349

Krug, H. B., Rupke, D. S. N., \& Veilleux, S. 2010, ApJ, 708, 1145

Kukula, M. J., Dunlop, J. S., Hughes, D. H., \& Rawlings, S. 1998, MNRAS, 297, 366

Lal, D. V., \& Ho, L. C. 2010, AJ, 139, 1089

Lavalley, M., Isobe, T., \& Feigelson, E. 1992, in Astronomical Data Analysis Software and Systems I, eds. D. M. Worrall, C. Biemesderfer, \& J. Barnes, ASP Conf. Ser., 25, 245

Lawrence, A. 1991, MNRAS, 252, 586

Leipski, C., \& Bennert, N. 2006, A\&A, 448, 165

Leipski, C., Falcke, H., Bennert, N., \& Hüttemeister, S. 2006, A\&A, 455, 161 
Letawe, G., Magain, P., Courbin, F., et al. 2007, MNRAS, 378, 83 Li, C., Kauffmann, G., Heckman, T. M., White, S. D. M., \& Jing, Y. P. 2008, MNRAS, 385, 1915

Lipari, S., Sanchez, S. F., Bergmann, M., et al. 2009, MNRAS, 392, 1295

Liu, X., Shen, Y., Strauss, M. A., \& Greene, J. E. 2010, ApJ, 708, 427

Magorrian, J., Tremaine, S., Richstone, D., et al. 1998, AJ, 115, 2285

Maiolino, R., Gallerani, S., Neri, R., et al. 2012, MNRAS, 425, L66

Márquez, I., \& Petitjean, P. 2003, in Rev. Mex. Astron. Astrofis. Conf. Ser. 16, eds. J. M. Rodriguez Espinoza, F. Garzon Lopez, \& V. Melo Martin, 135

Marziani, P., Zamanov, R. K., Sulentic, J. W., \& Calvani, M. 2003, MNRAS, 345,1133

Matsuoka, Y. 2012, ApJ, 750, 54

McCarthy, P. J., van Breugel, W., Spinrad, H., \& Djorgovski, S. 1987, ApJ, 321, L29

McCarthy, P. J., Spinrad, H., \& van Breugel, W. 1995, ApJS, 99, 27

McCarthy, P. J., Baum, S. A., \& Spinrad, H. 1996, ApJS, 106, 281

McGill, K. L., Woo, J.-H., Treu, T., \& Malkan, M. A. 2008, ApJ, 673, 703

McLeod, K. K., \& McLeod, B. A. 2001, ApJ, 546, 782

McLure, R. J., Kukula, M. J., Dunlop, J. S., et al. 1999, MNRAS, 308, 377

Miller, P., Rawlings, S., \& Saunders, R. 1993, MNRAS, 263, 425

Moorwood, A., Cuby, J., \& Lidman, C. 1998, The Messenger, 91,9

Mor, R., Netzer, H., \& Elitzur, M. 2009, ApJ, 705, 298

Morganti, R., Tadhunter, C. N., \& Oosterloo, T. A. 2005, A\&A, 444, L9

Mulchaey, J. S., Wilson, A. S., \& Tsvetanov, Z. 1996a, ApJS, 102, 309

Mulchaey, J. S., Wilson, A. S., \& Tsvetanov, Z. 1996b, ApJ, 467, 197

Müller-Sánchez, F., Prieto, M. A., Hicks, E. K. S., et al. 2011, ApJ, 739, 69

Nagar, N. M., Wilson, A. S., Mulchaey, J. S., \& Gallimore, J. F. 1999, ApJS, 120,209

Nenkova, M., Sirocky, M. M., Nikutta, R., Ivezić, Ž., \& Elitzur, M. 2008, ApJ, 685,160

Nesvadba, N. P. H., Lehnert, M. D., Eisenhauer, F., et al. 2006, ApJ, 650, 693

Netzer, H., Shemmer, O., Maiolino, R., et al. 2004, ApJ, 614, 558

Osterbrock, D. E., \& Ferland, G. J. 2006, Astrophysics of gaseous nebulae and active galactic nuclei, 2nd edn., eds. D. E. Osterbrock, \& G. J. Ferland (Sausalito, CA: University Science Books)

Peacock, J. A. 1983, MNRAS, 202, 615

Peng, C. Y., Ho, L. C., Impey, C. D., \& Rix, H.-W. 2002, AJ, 124, 266

Peng, C. Y., Ho, L. C., Impey, C. D., \& Rix, H. 2010, AJ, 139, 2097

Peterson, B. M., \& Wandel, A. 2000, ApJ, 540, L13

Peterson, B. M., Ferrarese, L., Gilbert, K. M., et al. 2004, ApJ, 613, 682

Pettini, M., \& Pagel, B. E. J. 2004, MNRAS, 348, L59

Press, W. H., Teukolsky, S. A., Vetterling, W. T., \& Flannery, B. P. 1992,

Numerical recipes in C. The art of scientific computing, 2nd edn. (Cambridge: University Press)

Privon, G. C., O'Dea, C. P., Baum, S. A., et al. 2008, ApJS, 175, 423

Punsly, B., \& Zhang, S. 2011, MNRAS, 412, L123

Pych, W. 2004, PASP, 116, 148

Rawlings, S., \& Saunders, R. 1991, Nature, 349, 138

Richards, G. T., Lacy, M., Storrie-Lombardi, L. J., et al. 2006, ApJS, 166, 470

Roth, M. M., Kelz, A., Fechner, T., et al. 2005, PASP, 117, 620

Rupke, D. S. N., \& Veilleux, S. 2011, ApJ, 729, L27

Rupke, D. S., Veilleux, S., \& Sanders, D. B. 2005, ApJS, 160, 115

Sánchez, S. F. 2006, AN, 327, 850

Sánchez, S. F., \& González-Serrano, J. I. 2003, A\&A, 406, 435

Sánchez, S. F., Garcia-Lorenzo, B., Mediavilla, E., González-Serrano, J. I., \&

Christensen, L. 2004a, ApJ, 615, 156
Sánchez, S. F., Jahnke, K., Wisotzki, L., et al. 2004b, ApJ, 614, 586

Sánchez, S. F., Aceituno, J., Thiele, U., Pérez-Ramírez, D., \& Alves, J. 2007, PASP, 119, 1186

Sanders, D. B., Soifer, B. T., Elias, J. H., Neugebauer, G., \& Matthews, K. 1988, ApJ, 328, L35

Schinnerer, E., Eckart, A., \& Tacconi, L. J. 1998, ApJ, 500, 147

Schlegel, D. J., Finkbeiner, D. P., \& Davis, M. 1998, ApJ, 500, 525

Schmidt, M., \& Green, R. F. 1983, ApJ, 269, 352

Schmitt, H. R., Donley, J. L., Antonucci, R. R. J., Hutchings, J. B., \& Kinney, A. L. 2003a, ApJS, 148, 327

Schmitt, H. R., Donley, J. L., Antonucci, R. R. J., et al. 2003b, ApJ, 597, 768

Schulze, A., \& Wisotzki, L. 2010, A\&A, 516, A87

Schulze, A., Wisotzki, L., \& Husemann, B. 2009, A\&A, 507, 781

Sersic, J. L. 1968, Atlas de galaxias australes, ed. J. L. Sersic

Smith, K. L., Shields, G. A., Bonning, E. W., et al. 2010, ApJ, 716, 866

Stasińska, G., Vale Asari, N., Cid Fernandes, R., et al. 2008, MNRAS, 391, L29

Steinhardt, C. L., \& Elvis, M. 2010, MNRAS, 402, 2637

Stockton, A. 1976, ApJ, 205, L113

Stockton, A., \& MacKenty, J. W. 1987, ApJ, 316, 584

Sulentic, J. W., Zwitter, T., Marziani, P., \& Dultzin-Hacyan, D. 2000, ApJ, 536, L5

Sullivan, M., Mobasher, B., Chan, B., et al. 2001, ApJ, 558, 72

Tadhunter, C., Wills, K., Morganti, R., Oosterloo, T., \& Dickson, R. 2001, MNRAS, 327, 227

Tremaine, S., Gebhardt, K., Bender, R., et al. 2002, ApJ, 574, 740

Tremonti, C. A., Heckman, T. M., Kauffmann, G., et al. 2004, ApJ, 613, 898

Tremonti, C. A., Moustakas, J., \& Diamond-Stanic, A. M. 2007, ApJ, 663, L77

van der Marel, R. P., \& Franx, M. 1993, ApJ, 407, 525

Veilleux, S., \& Osterbrock, D. E. 1987, ApJS, 63, 295

Veilleux, S., Kim, D., Rupke, D. S. N., et al. 2009, ApJ, 701, 587

Véron-Cetty, M., Joly, M., \& Véron, P. 2004, A\&A, 417, 515

Vestergaard, M. 2002, ApJ, 571, 733

Vestergaard, M., \& Peterson, B. M. 2006, ApJ, 641, 689

Villar-Martin, M., Tadhunter, C., \& Clark, N. 1997, A\&A, 323, 21

Villar-Martín, M., Tadhunter, C., Morganti, R., Axon, D., \& Koekemoer, A. 1999, MNRAS, 307, 24

Villar-Martín, M., Tadhunter, C., Pérez, E., et al. 2010, MNRAS, 407, L6

Villar-Martín, M., Humphrey, A., Delgado, R. G., Colina, L., \& Arribas, S. 2011a, MNRAS, 418, 2032

Villar-Martín, M., Tadhunter, C., Humphrey, A., et al. 2011b, MNRAS, 416, 262

Wampler, E. J., Burbidge, E. M., Baldwin, J. A., \& Robinson, L. B. 1975, ApJ, 198, L49

Wang, J.-M., Chen, Y.-M., Hu, C., et al. 2009, ApJ, 705, L76

Westmoquette, M. S., Clements, D. L., Bendo, G. J., \& Khan, S. A. 2012, MNRAS, 424, 416

Wild, V., Heckman, T., \& Charlot, S. 2010, MNRAS, 405, 933

Wilson, A. S., \& Tsvetanov, Z. I. 1994, AJ, 107, 1227

Wisotzki, L. 2000, A\&A, 353, 861

Wisotzki, L., Christlieb, N., Bade, N., et al. 2000, A\&A, 358, 77

Woo, J., Treu, T., Malkan, M. A., \& Blandford, R. D. 2006, ApJ, 645, 900

Wright, E. L. 2006, PASP, 118, 1711

Xu, C., Livio, M., \& Baum, S. 1999, AJ, 118, 1169

Xu, D., Komossa, S., Zhou, H., et al. 2012, AJ, 143, 83

Yang, J., Wu, F., Paragi, Z., \& An, T. 2012, MNRAS, 419, L74

Zakamska, N. L., Strauss, M. A., Krolik, J. H., et al. 2003, AJ, 126, 2125

Zamfir, S., Sulentic, J. W., \& Marziani, P. 2008, MNRAS, 387, 856

Pages 31 to 33 are available in the electronic edition of the journal at $h t t p: / /$ ww . aanda.org 
B. Husemann et al.: The properties of the extended warm ionised gas around low-redshift QSOs

Table 3. Broad-band imaging analysis results - structural parameters and morphological host galaxy classification.

\begin{tabular}{|c|c|c|c|c|c|c|c|c|c|}
\hline Name & Telescope/instrument & Ref. $^{a}$ & $\chi_{\nu}^{2}(n=1)$ & $\chi_{v}^{2}(n=4)$ & $\chi_{v}^{2}(n)$ & $r_{\mathrm{e}}^{b}$ & $b / a^{c}$ & $\mathrm{PA}^{d}$ & Class. $^{e}$ \\
\hline PG 0026+129 & HST NICMOS (F160W) & (1) & 4.400 & 3.566 & $3.563(3.6)$ & $1^{\prime \prime} .2(3.0 \mathrm{kpc})$ & 0.82 & $85^{\circ}$ & B \\
\hline PG $0050+124$ & HST WFPC2 (F814W) & & 2.826 & 2.684 & $2.669(2.8)$ & $8,7(10.2 \mathrm{kpc})$ & 0.73 & $-58^{\circ}$ & $\mathrm{B}+\mathrm{D}$ \\
\hline PG $0052+251$ & HST WFPC2 (F606W) & (2) & 2.705 & 3.368 & $2.704(1.0)$ & $3.5(9.4 \mathrm{kpc})$ & 0.65 & $-8^{\circ}$ & $\mathrm{D}$ \\
\hline \multirow[t]{2}{*}{ SDSS J0057+1446 } & $\operatorname{SDSS}\left(i^{\prime}\right)$ & & 1.002 & 0.990 & $0.989(2.7)$ & 3.' $7(10.8 \mathrm{kpc})$ & 0.52 & $-50^{\circ}$ & $\mathrm{B}$ ? \\
\hline & $\operatorname{SDSS}\left(r^{\prime}\right)$ & & 1.018 & 0.985 & $0.984(6.7)$ & $2^{\prime \prime} .2(6.4 \mathrm{kpc})$ & 0.62 & $-49^{\circ}$ & \\
\hline RBS 219 & LB Apogee U42 $(R)$ & & 1.505 & 1.508 & $1.505(1.1)$ & 3.'8 (10.1 kpc) & 0.79 & $28^{\circ}$ & $\mathrm{D}(\mathrm{V})$ \\
\hline \multirow[t]{2}{*}{ SDSS J0155-0857 } & $\operatorname{SDSS}\left(i^{\prime}\right)$ & & 1.180 & 1.150 & $1.148(5.4)$ & $1.3(3.4 \mathrm{kpc})$ & 0.62 & $32^{\circ}$ & B? \\
\hline & $\operatorname{SDSS}\left(r^{\prime}\right)$ & & 1.136 & 1.139 & $1.132(1.7)$ & $2 . \prime 4(6.8 \mathrm{kpc})$ & 0.57 & $31^{\circ}$ & \\
\hline PG $0157+001$ & HST WFPC2 (F675W) & (3) & 6.406 & 6.471 & $6.360(1.5)$ & 4.'9 (13.8 kpc) & 0.89 & $15^{\circ}$ & $\mathrm{D} / \mathrm{M}$ \\
\hline \multirow[t]{2}{*}{ SDSS J0836+4426 } & $\operatorname{SDSS}\left(i^{\prime}\right)$ & & 0.984 & 0.979 & $0.980(6.1)$ & $0.2(0.8 \mathrm{kpc})$ & 0.52 & $-83^{\circ}$ & $?$ \\
\hline & $\operatorname{SDSS}\left(r^{\prime}\right)$ & & 0.994 & 0.984 & $0.984(12.0)$ & $0.2(0.8 \mathrm{kpc})$ & 0.90 & $16^{\circ}$ & \\
\hline \multirow[t]{2}{*}{ SDSS J0948+4335 } & $\operatorname{SDSS}\left(i^{\prime}\right)$ & & 1.244 & 1.259 & $1.243(1.2)$ & $22^{\prime \prime} 4(8.7 \mathrm{kpc})$ & 0.80 & $-31^{\circ}$ & $\mathrm{D}(\mathrm{V})$ \\
\hline & $\operatorname{SDSS}\left(r^{\prime}\right)$ & & 1.052 & 1.083 & $1.051(0.8)$ & $2.7(9.8 \mathrm{kpc})$ & 0.79 & $-27^{\circ}$ & \\
\hline \multirow{2}{*}{ SDSS J1131+2632 } & $\operatorname{SDSS}\left(i^{\prime}\right)$ & & 1.213 & 1.255 & $1.212(0.8)$ & 3.'7 (14.2 kpc) & 0.74 & $-70^{\circ}$ & $\mathrm{M}(\mathrm{V})$ \\
\hline & $\operatorname{SDSS}\left(r^{\prime}\right)$ & & 1.111 & 1.174 & $1.105(0.7)$ & 3.' $4(13.1 \mathrm{kpc})$ & 0.75 & $-73^{\circ}$ & \\
\hline PKS $1217+023$ & HST WFPC2 (F675W) & (4) & 4.850 & 4.722 & $4.719(3.15)$ & $2.5(9.7 \mathrm{kpc})$ & 0.79 & $85^{\circ}$ & $\mathrm{B}$ \\
\hline $3 C 273$ & HST WFPC2 (F606W) & (5) & 1.941 & 1.823 & $1.814(2.8)$ & $5 .^{\prime} 0(13.7 \mathrm{kpc})$ & 0.80 & $78^{\circ}$ & $\mathrm{B}$ \\
\hline \multirow{2}{*}{ SDSS J1230+6621 } & $\operatorname{SDSS}\left(i^{\prime}\right)$ & & 1.340 & 1.340 & $1.339(1.7)$ & $1.8(5.6 \mathrm{kpc})$ & 0.85 & $15^{\circ}$ & $\mathrm{D} ?$ \\
\hline & $\operatorname{SDSS}\left(r^{\prime}\right)$ & & 1.047 & 1.052 & $1.047(1.1)$ & $1.8(5.6 \mathrm{kpc})$ & 0.73 & $24^{\circ}$ & \\
\hline \multirow[t]{2}{*}{ HE $1228+0131$} & $\operatorname{SDSS}\left(i^{\prime}\right)$ & & 1.038 & 1.006 & $0.992(9.9)$ & $0.3(0.6 \mathrm{kpc})$ & 0.66 & $-48^{\circ}$ & ? \\
\hline & $\operatorname{SDSS}\left(r^{\prime}\right)$ & & 1.122 & 1.110 & $1.104(9.0)$ & $0 . ' 8(1.7 \mathrm{kpc})$ & 0.68 & $-63^{\circ}$ & \\
\hline \multirow[t]{2}{*}{ SDSS J1230+1100 } & $\operatorname{SDSS}\left(i^{\prime}\right)$ & & 0.878 & 0.867 & $0.864(6.1)$ & $0.2(0.8 \mathrm{kpc})$ & 0.77 & $-86^{\circ}$ & $?$ \\
\hline & $\operatorname{SDSS}\left(r^{\prime}\right)$ & & 1.055 & 1.047 & $1.043(8.5)$ & $0.2(0.8 \mathrm{kpc})$ & 0.82 & $-30^{\circ}$ & \\
\hline \multirow{2}{*}{ SDSS J1444+0633 } & $\operatorname{SDSS}\left(i^{\prime}\right)$ & & 0.982 & 0.975 & $0.972(12.1)$ & $1^{\prime \prime} 3(4.4 \mathrm{kpc})$ & 0.68 & $85^{\circ}$ & ? \\
\hline & $\operatorname{SDSS}\left(r^{\prime}\right)$ & & 0.981 & 0.974 & $0.974(7.2)$ & $1.7(5.4 \mathrm{kpc})$ & 0.70 & $90^{\circ}$ & \\
\hline \multirow[t]{2}{*}{ PG $1427+480$} & $\operatorname{SDSS}\left(i^{\prime}\right)$ & & 1.438 & 1.408 & $\ldots$ & $0 .^{\prime} 9(3.2 \mathrm{kpc})$ & 0.90 & $34^{\circ}$ & $\mathrm{B}$ ? \\
\hline & $\operatorname{SDSS}\left(r^{\prime}\right)$ & & 1.039 & 1.033 & $1.032(6.8)$ & $0.6(2.1 \mathrm{kpc})$ & 0.79 & $20^{\circ}$ & \\
\hline PKS $1545+210$ & HST WFPC2 (F606W) & (5) & 2.667 & 2.606 & $2.603(3.0)$ & $1^{\prime \prime} 8(7.4 \mathrm{kpc})$ & 0.85 & $77^{\circ}$ & B \\
\hline \multirow{2}{*}{ PG $1612+261$} & $\operatorname{SDSS}\left(i^{\prime}\right)$ & & 1.057 & 1.053 & $1.047(2.2)$ & $1^{\prime \prime} 6(3.7 \mathrm{kpc})$ & 0.37 & $10^{\circ}$ & $\mathrm{D}(\mathrm{V})$ \\
\hline & $\operatorname{SDSS}\left(r^{\prime}\right)$ & & 1.057 & 1.068 & $1.047(1.7)$ & $1^{\prime \prime} 7(3.96 \mathrm{kpc})$ & 0.40 & $11^{\circ}$ & \\
\hline \multirow[t]{2}{*}{ SDSS J1655+2146 } & $\operatorname{SDSS}\left(i^{\prime}\right)$ & & 1.795 & 1.662 & $\ldots$ & $0.2(0.5 \mathrm{kpc})$ & 0.94 & $51^{\circ}$ & $?$ \\
\hline & $\operatorname{SDSS}\left(r^{\prime}\right)$ & & 1.188 & 1.140 & $\ldots$ & $0 .{ }^{\prime} 6(1.6 \mathrm{kpc})$ & 0.82 & $30^{\circ}$ & \\
\hline PG $1700+518$ & HST WFPC2 (F547M) & (1) & 1.800 & 1.742 & & $1^{\prime \prime} 4(6.2 \mathrm{kpc})$ & 0.80 & $23^{\circ}$ & $\mathrm{M}(\mathrm{V})$ \\
\hline \multirow[t]{2}{*}{ PG 2130+099 } & HST WFPC2 (F606W) & (6) & 1.870 & 1.875 & $1.833(1.8)$ & $12^{\prime \prime} 0(14 \mathrm{kpc})$ & 0.36 & $60^{\circ}$ & $\mathrm{B}+\mathrm{D}$ \\
\hline & NTT SOFI $(H)$ & & 1.417 & 1.372 & $1.360(2.4)$ & $7 " .3(8.6 \mathrm{kpc})$ & 0.31 & $51^{\circ}$ & \\
\hline \multirow[t]{3}{*}{ PHL 1811} & HST ACS (F625W) & (7) & 1.591 & 1.552 & $\ldots$ & $1^{\prime \prime} 7(5.3 \mathrm{kpc})$ & 0.52 & $72^{\circ}$ & $\mathrm{D}(\mathrm{V})$ \\
\hline & NTT SOFI $(H)$ & & 1.156 & 1.128 & $1.091(9.6)$ & $0.7(2.2 \mathrm{kpc})$ & 0.48 & $59^{\circ}$ & \\
\hline & LB Apogee U42 $(R)$ & & 1.263 & 1.248 & $1.243(8.5)$ & $1^{\prime \prime} .7(5.3 \mathrm{kpc})$ & 0.59 & $60^{\circ}$ & \\
\hline \multirow[t]{2}{*}{ HE $2158-0107^{f}$} & $\operatorname{SDSS}(z)$ & & 0.884 & 0.880 & $0.880(6.2)$ & $0.2(0.7 \mathrm{kpc})$ & 0.93 & $43^{\circ}$ & $\mathrm{B}$ ? \\
\hline & NTT SOFI $(H)$ & & $\ldots$ & $\ldots$ & $\ldots$ & $\ldots$ & $\ldots$ & $\ldots$ & \\
\hline \multirow{3}{*}{ PG 2214+139 } & NTT SOFI $(H)$ & & 1.014 & 1.063 & $0.950(1.8)$ & $2^{\prime \prime} 9(3.7 \mathrm{kpc})$ & 0.97 & $64^{\circ}$ & B \\
\hline & $\operatorname{SDSS}\left(i^{\prime}\right)$ & & 1.825 & 1.380 & $1.299(5.4)$ & $4{ }^{\prime \prime} 2(5.4 \mathrm{kpc})$ & 0.94 & $63^{\circ}$ & \\
\hline & $\operatorname{SDSS}\left(r^{\prime}\right)$ & & 2.326 & 1.814 & $1.684(5.8)$ & $4^{\prime \prime} 1(5.3 \mathrm{kpc})$ & 0.94 & $56^{\circ}$ & \\
\hline HE 2307-0254 & NTT SOFI $(H)$ & & 0.542 & 0.542 & $0.542(6.2)$ & $0.2(0.7 \mathrm{kpc})$ & 0.67 & $35^{\circ}$ & $\mathrm{B}$ ? \\
\hline PKS 2349-014 & HST WFPC2 (F606W) & (8) & 2.649 & 2.441 & $2.436(3.3)$ & 5.' $6(16.5 \mathrm{kpc})$ & 0.81 & $83^{\circ}$ & $\mathrm{B} / \mathrm{M}$ \\
\hline HE $2353-0420$ & LB Apogee U42 $(R)$ & & 0.940 & 0.957 & $0.939(0.7)$ & $3{ }^{\prime \prime} 8(13.9 \mathrm{kpc})$ & 0.49 & $15^{\circ}$ & $\mathrm{D}(\mathrm{V})$ \\
\hline
\end{tabular}

Notes. ${ }^{(a)}$ References to the articles in which a morphological analysis of these data was initally presented. ${ }^{(b)}$ Effective radius $r_{\mathrm{e}}$ of the best-fitting realistic host-galaxy model given in apparent and physical scale. ${ }^{(c)}$ Axis ratio of the minor to major axis of the best-fitting model. ${ }^{(d)}$ Position angle of the major axis of the best-fitting model oriented such that $0^{\circ}$ is North and $90^{\circ}$ is East. ${ }^{(e)}$ Our host galaxy morphological classification into: B (bulge-dominated), D (disc-dominated), B+D (bulge+disc component), M (Merger or strongly interacting), B?/D? (only tentative classifications), ? (unconstrained). We append (V) to the classification, if it is due to a visual inspection of the image. (f) Unresolved host galaxy.

References. (1) Veilleux et al. (2009); (2) Bahcall et al. (1996); (3) McLure et al. (1999); (4) Dunlop et al. (2003); (5) Bahcall et al. (1997); (6) Kim et al. (2008); (7) Jenkins et al. (2005); (8) Bahcall et al. (1995). 
Table 4. PMAS IFU observational log.

\begin{tabular}{|c|c|c|c|c|c|c|}
\hline Object & Obs. date & Exp. time [s] & Grating & Wavelength range $(\AA)$ & Seeing & Obs. condition \\
\hline PG 0026+129 & 2002-09-05 & $1 \times 1200,1 \times 2400$ & V300 & $4600-7800$ & $1 " .1$ & photometric \\
\hline I Zw 1 & 2002-09-03 & $1 \times 900,2 \times 1800$ & V300 & $3900-7200$ & $1^{\prime \prime} 0-1 . .4$ & mainly clear \\
\hline PG $0052+251$ & 2002-09-06 & $2 \times 1800$ & V300 & $4600-7800$ & $1^{\prime \prime} 0$ & photometric \\
\hline SDSS J0057+1446 & 2008-09-05 & $5 \times 1800$ & V600 & $5100-6600$ & $1 . .5$ & part. cloudy \\
\hline RBS 219 & 2002-09-02 & $1 \times 1200,1 \times 1800$ & V300 & $4600-7800$ & 1 1". $2-1$ ". 8 & photometric \\
\hline SDSS J0155-0857 & 2008-09-07 & $\begin{array}{l}4 \times 1800 \\
2 \times 1800\end{array}$ & V600 & $5100-6600$ & $\begin{array}{l}\text { 1.. } 4-1 \text {-1. } 8 \\
\text { 1.. } 2\end{array}$ & $\begin{array}{l}\text { photometric } \\
\text { photometric }\end{array}$ \\
\hline HE 0157-0406 & 2002-09-02 & $1 \times 1200,1 \times 1800$ & V300 & $5400-8600$ & $1 " .2-1 . \prime 3$ & photometric \\
\hline \multirow[t]{2}{*}{ Mrk 1014} & 2002-09-07 & $1 \times 1200,1 \times 1800$ & V300 & $5400-8600$ & $1^{\prime \prime} 2-1^{\prime \prime} 3$ & photometric \\
\hline & 2008-09-06 & $4 \times 1800$ & V600 & $5100-6600$ & $1{ }^{\prime \prime} 4$ & mainly clear \\
\hline SDSS J0836+4426 & 2008-03-16 & $3 \times 1800$ & V600 & $5500-7000$ & $1 . .1$ & part. cloudy \\
\hline SDSS J0948+4335 & 2008-03-13 & $3 \times 1800$ & V600 & $5250-6750$ & $1 . \prime 1$ & cloudy \\
\hline SDSS J1131+2623 & 2008-03-13 & $3 \times 1800$ & V600 & $5250-6750$ & $1^{\prime \prime} 1$ & part. cloudy \\
\hline PKS 1217+023 & 2003-05-02 & $4 \times 1800$ & V300 & $5600-8900$ & $1^{\prime \prime} 0-11^{\prime \prime} 2$ & mainly clear \\
\hline $3 \mathrm{C} 273$ & 2003-05-03 & $3 \times 1200$ & V300 & $4350-7650$ & $1 " .4-1 . \prime 9$ & mainly clear \\
\hline HE $1228+0131$ & 2003-05-04 & $4 \times 1800$ & V300 & $4350-7650$ & $1{ }^{\prime \prime} 0-1 . .3$ & part. cloudy \\
\hline SDSS J1230+6621 & $2008-03-16$ & $2 \times 1800$ & V600 & $5000-6500$ & 1 ." 0 & mainly clear \\
\hline SDSS J1230+1100 & 2008-03-13 & $1 \times 1800$ & V600 & $5250-6750$ & $1 . " 1$ & cloudy \\
\hline PG $1427+490$ & 2003-05-01 & $3 \times 1200$ & V300 & $5600-8900$ & $0.9-1{ }^{\prime \prime} 2$ & mainly clear \\
\hline SDSS J1444+0633 & $2008-03-16$ & $1 \times 1800$ & V600 & $5250-6750$ & $1^{\prime \prime} 3-1^{\prime \prime} 5$ & cloudy \\
\hline \multirow{2}{*}{ HE $1453-0303$} & 2003-05-02 & $2 \times 1800$ & V300 & $5600-8900$ & 1..2-1". 5 & mainly clear \\
\hline & 2003-05-03 & $2 \times 1800$ & & & $1 " .3-1 . .6$ & mainyl clear \\
\hline PKS 1545+21 & $2003-05-03$ & $3 \times 1800$ & V300 & $5600-8900$ & $1 " .2-1 " .5$ & mainly clear \\
\hline PG $1612+261$ & 2008-09-07 & $3 \times 1800$ & V600 & $5100-6600$ & 1 .. 1 & mainly clear \\
\hline \multirow[t]{2}{*}{ SDSS J1655+2146 } & 2008-03-16 & $3 \times 1200$ & V600 & $5000-6500$ & 1.. $3-1 " .6$ & part. cloudy \\
\hline & & $1 \times 1800$ & & & $1{ }^{\prime \prime} 0$ & photometric \\
\hline \multirow[t]{2}{*}{ PG $1700+518$} & 2003-05-02 & $2 \times 1800$ & V300 & $5600-8900$ & $0.9-11^{\prime \prime} 2$ & photometric \\
\hline & 2003-05-03 & $2 \times 1800$ & & & 1 .. 6 & mainly clear \\
\hline PG 2130+099 & 2002-09-04 & $2 \times 2400$ & V300 & $3900-7200$ & 1.3 & photometric \\
\hline PHL 1811 & 2002-09-06 & $2 \times 1800$ & V300 & $5400-8600$ & 1."2 & photometric \\
\hline HE $2158-0107$ & 2002-09-06 & $2 \times 1800$ & V300 & $5400-8600$ & 1." 1 & photometric \\
\hline \multirow[t]{2}{*}{ RBS 1812} & 2002-09-04 & $1 \times 2400$ & V300 & $4600-7800$ & 1 1. 5 & photometric \\
\hline & 2002-09-05 & $2 \times 2400$ & & & 1..6-2". 0 & mainly clear \\
\hline PG 2214+139 & 2002-09-02 & $2 \times 2400,1 \times 1800$ & V300 & $3900-7200$ & $1^{\prime \prime} 3-11^{\prime \prime} 4$ & photometric \\
\hline HE $2307-0254$ & 2002-09-06 & $2 \times 1800$ & V300 & $5400-8600$ & $1 " .2$ & photometric \\
\hline PKS 2349-014 & 2002-09-06 & $3 \times 900$ & V300 & $5400-8600$ & $11^{\prime \prime} 0-1{ }^{\prime \prime} 1$ & photometric \\
\hline HE $2353-0420$ & 2002-09-07 & $2 \times 1800$ & V300 & $5400-8600$ & $1^{\prime \prime} 1-11^{\prime \prime} 4$ & photometric \\
\hline
\end{tabular}


B. Husemann et al.: The properties of the extended warm ionised gas around low-redshift QSOs

Table 7. Kinematics and line fluxes for specific regions of the EELR.

\begin{tabular}{|c|c|c|c|c|c|c|c|c|}
\hline \multirow[t]{2}{*}{ Object $^{a}$} & & \multirow{2}{*}{$\begin{array}{c}\Delta v_{\mathrm{r}}^{b} \\
{\left[\mathrm{~km} \mathrm{~s}^{-1}\right]}\end{array}$} & \multirow{2}{*}{$\begin{array}{c}\sigma_{\text {line }^{c}} \\
{\left[\mathrm{~km} \mathrm{~s}^{-1}\right]}\end{array}$} & $\mathrm{H} \beta$ & {$[\mathrm{O}$ III $] \lambda 5007$} & $\mathrm{H} \alpha$ & {$[\mathrm{N}$ II] $] \lambda 6585$} & \multirow[t]{2}{*}{ Classification } \\
\hline & & & & \multicolumn{4}{|c|}{$\left[10^{-16} \mathrm{erg} \mathrm{s}^{-1} \mathrm{~cm}^{-2}\right]$} & \\
\hline PG 0026+129 & $\begin{array}{l}\text { A } \\
\mathrm{B}\end{array}$ & $\begin{array}{c}48 \pm 34 \\
136 \pm 32\end{array}$ & $\begin{array}{c}158 \pm 14 \\
153 \pm 9\end{array}$ & $\begin{array}{l}2.4 \pm 0.9 \\
2.5 \pm 0.4\end{array}$ & $\begin{array}{l}25.1 \pm 1.0 \\
19.8 \pm 0.5\end{array}$ & $\begin{array}{l}\cdots \\
\cdots\end{array}$ & $\begin{array}{l}\cdots \\
\ldots\end{array}$ & $\begin{array}{l}\text { AGN } \\
\text { AGN }\end{array}$ \\
\hline PG $0050+124$ & $\begin{array}{l}\text { A } \\
\text { B } \\
\text { C }\end{array}$ & $\begin{array}{l}-312 \pm 27 \\
-475 \pm 28 \\
-215 \pm 36\end{array}$ & $\begin{array}{l}83 \pm 24 \\
18 \pm 23 \\
80 \pm 30\end{array}$ & $\begin{array}{l}\ldots \\
\ldots \\
\ldots\end{array}$ & $\begin{array}{l}\ldots \\
\ldots \\
\ldots\end{array}$ & $\begin{array}{l}9.3 \pm 1.4 \\
6.9 \pm 2.8 \\
9.8 \pm 1.9\end{array}$ & $\begin{array}{l}7.9 \pm 0.3 \\
7.7 \pm 0.4 \\
5.8 \pm 0.7\end{array}$ & $\begin{array}{l}\text { H II } \\
\text { H II } \\
\text { H II }\end{array}$ \\
\hline PG $0052+251$ & $\begin{array}{l}\text { A } \\
\text { B } \\
\text { C }\end{array}$ & $\begin{array}{l}-127 \pm 36 \\
-232 \pm 32 \\
-264 \pm 41\end{array}$ & $\begin{array}{c}110 \pm 31 \\
82 \pm 27 \\
65 \pm 28\end{array}$ & $\begin{array}{l}3.0 \pm 0.5 \\
6.3 \pm 1.1 \\
5.5 \pm 1.2\end{array}$ & $\begin{array}{c}9.4 \pm 0.2 \\
38.5 \pm 0.2 \\
7.0 \pm 0.4\end{array}$ & $\begin{array}{c}9.5 \pm 0.4 \\
22.1 \pm 0.8 \\
14.1 \pm 1.1\end{array}$ & $\begin{array}{l}<2.5 \\
<3.4 \\
<5.3\end{array}$ & $\begin{array}{l}\text { H II } \\
\text { H II? } \\
\text { AGN }\end{array}$ \\
\hline RBS 219 & $\begin{array}{l}\text { A } \\
\text { B } \\
\text { C }\end{array}$ & $\begin{array}{c}-95 \pm 40 \\
17 \pm 39 \\
-105 \pm 47\end{array}$ & $\begin{array}{c}76 \pm 22 \\
0 \pm 25 \\
0 \pm 39\end{array}$ & $\begin{array}{l}\ldots \\
\ldots \\
\ldots\end{array}$ & $\begin{array}{l}\ldots \\
\ldots \\
\ldots\end{array}$ & $\begin{array}{c}12.1 \pm 1.2 \\
5.8 \pm 1.7 \\
4.7 \pm 2.9\end{array}$ & $\begin{array}{l}<2.5 \\
<4.6 \\
<8.3\end{array}$ & $\begin{array}{l}\text { H II } \\
\text { H II } \\
\text { H II }\end{array}$ \\
\hline SDSS J0155-0857 & $\begin{array}{l}\mathrm{A} \\
\mathrm{B}\end{array}$ & $\begin{array}{c}134 \pm 40 \\
-7 \pm 42\end{array}$ & $\begin{array}{c}73 \pm 12 \\
129 \pm 13\end{array}$ & $\begin{array}{l}0.4 \pm 0.3 \\
1.7 \pm 0.8\end{array}$ & $\begin{array}{l}3.5 \pm 0.3 \\
7.0 \pm 0.6\end{array}$ & $\begin{array}{l}\ldots \\
\ldots\end{array}$ & $\begin{array}{l}\ldots \\
\ldots\end{array}$ & $\begin{array}{l}\text { AGN } \\
\text { AGN }\end{array}$ \\
\hline Mrk 1014 & $\begin{array}{l}\text { A } \\
\text { B } \\
\text { C }\end{array}$ & $\begin{array}{c}-100 \pm 28 \\
67 \pm 32 \\
-222 \pm 31\end{array}$ & $\begin{array}{l}155 \pm 22 \\
229 \pm 17 \\
113 \pm 28\end{array}$ & $\begin{array}{c}9.1 \pm 1.8 \\
14.0 \pm 3.5 \\
5.0 \pm 1.0\end{array}$ & $\begin{array}{l}53.9 \pm 0.5 \\
40.1 \pm 0.9 \\
23.0 \pm 0.5\end{array}$ & $\begin{array}{c}26.1 \pm 5.1^{d} \\
40.2 \pm 10.0^{d} \\
14.2 \pm 2.7^{d}\end{array}$ & $\begin{array}{c}14.6 \pm 0.7 \\
14.2 \pm 0.8 \\
6.8 \pm 1.0\end{array}$ & $\begin{array}{l}\text { AGN } \\
\text { AGN } \\
\text { AGN }\end{array}$ \\
\hline SDSS J0846+4426 & $\begin{array}{l}\text { A } \\
\text { B } \\
\text { C }\end{array}$ & $\begin{array}{c}-88 \pm 46 \\
-229 \pm 46 \\
-154 \pm 50\end{array}$ & $\begin{array}{l}132 \pm 3 \\
143 \pm 3 \\
130 \pm 8\end{array}$ & $\begin{array}{c}18.2 \pm 2.2 \\
14.2 \pm 1.4 \\
\quad<5.4\end{array}$ & $\begin{array}{c}198.2 \pm 2.3 \\
146.0 \pm 1.5 \\
58.6 \pm 1.8\end{array}$ & $\begin{array}{l}\ldots \\
\ldots \\
\ldots\end{array}$ & $\begin{array}{l}\ldots \\
\ldots \\
\ldots\end{array}$ & $\begin{array}{l}\text { AGN } \\
\text { AGN } \\
\text { AGN }\end{array}$ \\
\hline SDSS J1131+2623 & $\begin{array}{l}\text { A } \\
\text { B } \\
\text { C } \\
\text { D }\end{array}$ & $\begin{array}{c}-44 \pm 69 \\
-32 \pm 68 \\
-424 \pm 72 \\
131 \pm 76\end{array}$ & $\begin{array}{c}117 \pm 3 \\
87 \pm 4 \\
54 \pm 8 \\
105 \pm 10\end{array}$ & $\begin{array}{c}1.9 \pm 0.9 \\
1.6 \pm 0.3 \\
<1.3 \\
<2.4\end{array}$ & $\begin{array}{c}32.6 \pm 0.6 \\
25.7 \pm 0.4 \\
8.6 \pm 0.4 \\
13.9 \pm 0.8\end{array}$ & $\begin{array}{l}\ldots \\
\ldots \\
\ldots \\
\ldots\end{array}$ & $\begin{array}{l}\cdots \\
\cdots \\
\ldots \\
\ldots\end{array}$ & $\begin{array}{l}\text { AGN } \\
\text { AGN } \\
\text { AGN } \\
\text { AGN }\end{array}$ \\
\hline PKS 1217+023 & $\begin{array}{l}\mathrm{A} \\
\mathrm{B}\end{array}$ & $\begin{array}{l}45 \pm 35 \\
-61 \pm 37\end{array}$ & $\begin{array}{l}75 \pm 27 \\
80 \pm 27\end{array}$ & $\begin{array}{l}2.5 \pm 0.6 \\
3.4 \pm 1.1\end{array}$ & $\begin{array}{l}9.3 \pm 0.3 \\
19.4 \pm 0.7\end{array}$ & $\begin{array}{c}\cdots \\
6.1 \pm 0.8\end{array}$ & $\begin{array}{c}\cdots \\
2.8 \pm 0.5\end{array}$ & $\begin{array}{l}\text { AGN } \\
\text { AGN }\end{array}$ \\
\hline SDSS J1230+6621 & $\begin{array}{l}\text { A } \\
\text { B }\end{array}$ & $\begin{array}{c}299 \pm 24 \\
67 \pm 23\end{array}$ & $\begin{array}{c}114 \pm 6 \\
80 \pm 5\end{array}$ & $\begin{array}{l}4.0 \pm 0.9 \\
2.9 \pm 0.4\end{array}$ & $\begin{array}{l}35.3 \pm 0.9 \\
19.5 \pm 0.5\end{array}$ & $\begin{array}{l}\ldots \\
\ldots\end{array}$ & $\begin{array}{l}\ldots \\
\ldots\end{array}$ & $\begin{array}{l}\text { AGN } \\
\text { AGN }\end{array}$ \\
\hline PKS 1545+210 & $\begin{array}{l}\text { A } \\
\text { B }\end{array}$ & $\begin{array}{c}309 \pm 47 \\
-134 \pm 64\end{array}$ & $\begin{array}{c}93 \pm 29 \\
212 \pm 51\end{array}$ & $\begin{array}{l}4.5 \pm 2.4 \\
6.0 \pm 4.1\end{array}$ & $\begin{array}{l}36.9 \pm 1.8 \\
35.5 \pm 4.7\end{array}$ & $\begin{array}{l}\cdots \\
\cdots\end{array}$ & $\begin{array}{l}\cdots \\
\cdots\end{array}$ & $\begin{array}{l}\text { AGN } \\
\text { AGN }\end{array}$ \\
\hline PG $1612+261$ & $\begin{array}{l}\text { A } \\
\text { B } \\
\text { C } \\
\text { D }\end{array}$ & $\begin{array}{c}70 \pm 29 \\
54 \pm 28 \\
248 \pm 28 \\
-92 \pm 33\end{array}$ & $\begin{array}{c}77 \pm 5 \\
91 \pm 3 \\
82 \pm 4 \\
105 \pm 8\end{array}$ & $\begin{array}{c}<18.9 \\
<8.2 \\
18.2 \pm 3.7 \\
<4.8\end{array}$ & $\begin{array}{c}111.0 \pm 4.7 \\
289.1 \pm 2.0 \\
199.8 \pm 1.2 \\
29.3 \pm 1.2\end{array}$ & $\begin{array}{l}\ldots \\
\ldots \\
\ldots \\
\ldots\end{array}$ & $\begin{array}{l}\cdots \\
\cdots \\
\cdots \\
\ldots\end{array}$ & $\begin{array}{l}\text { AGN } \\
\text { AGN } \\
\text { AGN } \\
\text { AGN }\end{array}$ \\
\hline SDSS J1655+2146 & $\begin{array}{l}\text { A } \\
\text { B } \\
\text { C } \\
\text { D }\end{array}$ & $\begin{array}{c}-85 \pm 26 \\
-85 \pm 27 \\
-78 \pm 31 \\
-128 \pm 30\end{array}$ & $\begin{array}{c}161 \pm 2 \\
183 \pm 3 \\
197 \pm 7 \\
94 \pm 5\end{array}$ & $\begin{array}{c}1.3 \pm 0.8 \\
20.4 \pm 2.6 \\
8.1 \pm 1.0 \\
<4.1\end{array}$ & $\begin{array}{c}127.9 \pm 0.9 \\
319.3 \pm 3.0 \\
44.3 \pm 1.2 \\
43.5 \pm 1.4\end{array}$ & $\begin{array}{l}\cdots \\
\cdots \\
\cdots \\
\cdots\end{array}$ & $\begin{array}{l}\cdots \\
\cdots \\
\cdots \\
\cdots\end{array}$ & $\begin{array}{l}\text { AGN } \\
\text { AGN } \\
\text { AGN } \\
\text { AGN }\end{array}$ \\
\hline PG $1700+518$ & A & $-889 \pm 67$ & $158 \pm 39$ & $2.1 \pm 0.6$ & $9.2 \pm 0.8$ & $\ldots$ & $\ldots$ & AGN \\
\hline PG 2130+099 & $\begin{array}{l}\text { A } \\
\text { B }\end{array}$ & $\begin{array}{c}-99 \pm 68 \\
-262 \pm 36\end{array}$ & $\begin{array}{c}180 \pm 105 \\
176 \pm 32\end{array}$ & $\begin{array}{c}5.9 \pm 3.0 \\
10.1 \pm 2.8\end{array}$ & $\begin{array}{c}3.5 \pm 0.7 \\
22.1 \pm 1.3\end{array}$ & $\begin{array}{c}7.7 \pm 1.6 \\
21.6 \pm 1.1\end{array}$ & $\begin{array}{l}3.8 \pm 0.4 \\
9.5 \pm 0.3\end{array}$ & $\begin{array}{c}\text { H II } \\
\text { AGN? }\end{array}$ \\
\hline PHL 1811 & A & $49 \pm 27$ & $33 \pm 15$ & $3.4 \pm 0.4$ & $3.4 \pm 0.4$ & $14.6 \pm 1.0$ & $5.7 \pm 0.5$ & H II \\
\hline HE 2158-0107 & $\begin{array}{l}\text { A } \\
\text { B } \\
\text { C }\end{array}$ & $\begin{array}{c}42 \pm 44 \\
-50 \pm 39 \\
-50 \pm 47\end{array}$ & $\begin{array}{c}69 \pm 26 \\
39 \pm 23 \\
0 \pm 30\end{array}$ & $\begin{array}{c}8.1 \pm 1.4 \\
11.2 \pm 1.8 \\
\quad<3.6\end{array}$ & $\begin{array}{c}39.6 \pm 1.5 \\
108.5 \pm 1.9 \\
18.7 \pm 1.2\end{array}$ & $\begin{array}{c}11.5 \pm 1.6 \\
28.4 \pm 3.6 \\
\ldots\end{array}$ & $\begin{array}{c}<3.8 \\
<8.2 \\
\ldots\end{array}$ & $\begin{array}{l}\text { AGN } \\
\text { AGN } \\
\text { AGN }\end{array}$ \\
\hline PKS 2349-014 & $\begin{array}{l}\text { A } \\
\text { B } \\
\text { C } \\
\text { D }\end{array}$ & $\begin{array}{c}-33 \pm 49 \\
-269 \pm 57 \\
97 \pm 39 \\
418 \pm 47\end{array}$ & $\begin{array}{c}172 \pm 24 \\
299 \pm 21 \\
146 \pm 22 \\
0 \pm 26\end{array}$ & $\begin{array}{c}4.2 \pm 0.8 \\
5.6 \pm 2.1 \\
12.2 \pm 1.2 \\
1.3 \pm 0.1\end{array}$ & $\begin{array}{c}6.9 \pm 0.3 \\
20.8 \pm 1.1 \\
96.6 \pm 0.9 \\
5.8 \pm 0.3\end{array}$ & $\begin{array}{c}10.3 \pm 0.7 \\
11.7 \pm 2.2 \\
34.0 \pm 2.2 \\
\ldots\end{array}$ & $\begin{array}{c}5.7 \pm 0.4 \\
13.2 \pm 1.2 \\
36.8 \pm 1.6 \\
\ldots\end{array}$ & $\begin{array}{l}\text { H II } \\
\text { AGN } \\
\text { AGN } \\
\text { AGN }\end{array}$ \\
\hline HE 2353-0420 & $\begin{array}{l}\mathrm{A} \\
\mathrm{B}\end{array}$ & $\begin{array}{l}85 \pm 51 \\
66 \pm 46\end{array}$ & $\begin{array}{l}225 \pm 17 \\
189 \pm 18\end{array}$ & $\begin{array}{l}7.6 \pm 1.5 \\
6.0 \pm 1.3\end{array}$ & $\begin{array}{c}77.9 \pm 2.0 \\
116.5 \pm 1.8\end{array}$ & $\begin{array}{l}22.5 \pm 1.6 \\
32.5 \pm 2.2\end{array}$ & $\begin{array}{l}18.0 \pm 1.0 \\
26.6 \pm 1.6\end{array}$ & $\begin{array}{l}\text { AGN } \\
\text { AGN }\end{array}$ \\
\hline
\end{tabular}

Notes. ${ }^{(a)}$ Name of QSOs followed with the alphabetically ordered character of the specific co-added spectra. ${ }^{(b)}$ Radial velocity offset with respect to the rest-frame of the nuclear [O III] line. Negative values corresponds to a blue shift. ${ }^{(c)}$ Emission line dispersion after the instrumental dispersion was subtracted in quadrature. A zero dispersion means that the line was unresolved. ${ }^{(d)}$ The $\mathrm{H} \alpha$ line was strongly affected by telluric absorption and its flux was estimates from the $\mathrm{H} \beta$ line assuming zero dust extinction $(\mathrm{H} \alpha / \mathrm{H} \beta=2.87)$. 